\title{
THE EFFECTS OF FATIGUE AND ALCOHOL ON HIGHWAY SAFETY
}

\author{
Joan M. Ryder \\ Stacy A. Malin \\ Craig H. Kinsley \\ Koba Associates, Inc. \\ 2000 Florida Avenue, N.W. \\ Washington, D.C. 20009
}

Contract No. DTNH-22-80-C-07053

Contract Amt. $\$ 63,815$

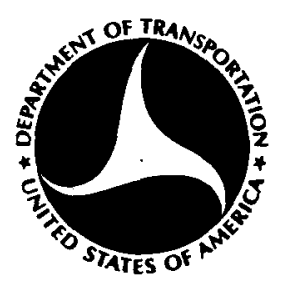

\section{MARCH 1981 FINAL REPORT}

This document is available to the U.S. public through the National Technical Information Service.

Springfield, Virginia 22161

\author{
Prepared For \\ U.S. DEPARTMENT OF TRANSPORTATION \\ National Highway Traffic Safety Administration \\ Washington, D.C. 20590
}


This document is disseminated under the sponsorship of the Department of Transportation in the interest of information exchange. The United States Government assumes no liability for 1ts contents or use thereof. 
Technical Report Documentation Page

1. Report No.
DoT-HS-805-854

3. Recipient's Cotolog No.

5. Repoil Dote

March, 1981

6. Performing Organization Code

8. Performing Orgonization Report No.

10. Work Unit No. (TRAIS)

11. Contract or Grant No.

DTNH-22-80-C-07053

13. Type of Report and Period Covered

Final Report

March 1980-March 1981

74. Sponsoring Agency Code

15. Supplementary Notes

16. Abstruct

The report presents a critical review of recent research, an assessment of present knowledge, and recommendations for future research to determine the combined effects of fatigue and alcohol on highway safety. The review concentrates on four areas: 1) definitions and measures of fatique; 2) experimental investigations of the effects of alcohol alone, fatigue alone, and alcohol and fatigue in combination on driver performance; 3) epidemiological studies which estimated the proportions of accidents caused by alcohol, fatigue, or both; and 4) possible remedies that have been suggested to counteract fatiguerelated driving impairments. The review found evidence suggesting that fatigue may exacerbate the impairing effects of moderate or large amounts of alcohol. However, the state of knowledge in this area was found to be incomplete, and further research will be required to draw specific conclusions. Recommendations for the direction of future research are presented.

17. l.ey Words
Driver Performance
Fatigue
Alcohol

19. Security Classit. (of this report)

Unclassified

Form DOT F $1700.7(8-72)$

\section{Alcohol}

18. Distribution Statement

Document is available to the U.S. public through the National Technical Information Service, Springfield, Virginia 22161 
MEtaic CONVERSION factors
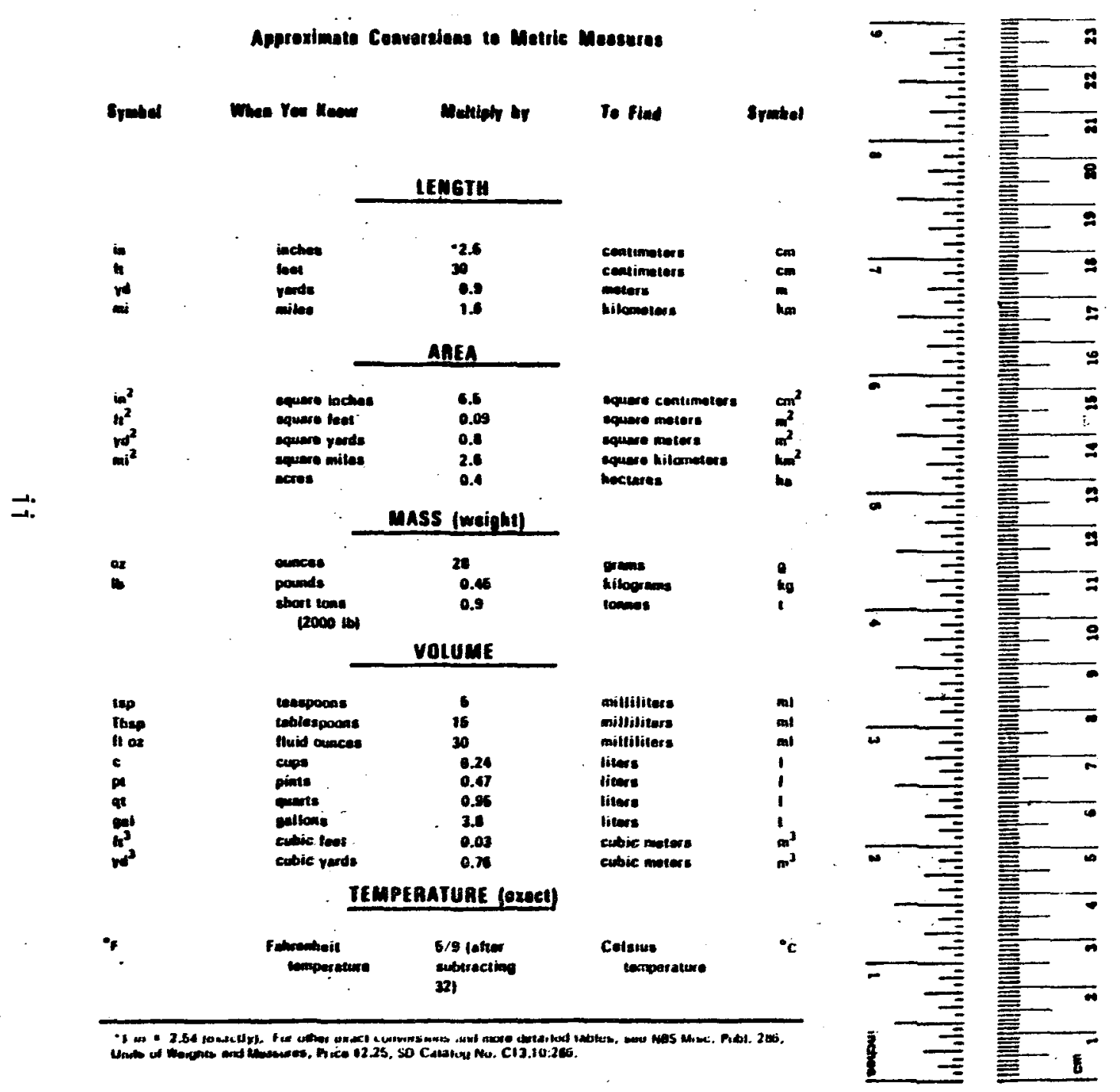

srabal

Appronimate Ceaversions from Motric Mosures

$i_{i \infty}^{\infty}$

when You kaow

Muttialy by

Io find

Srated

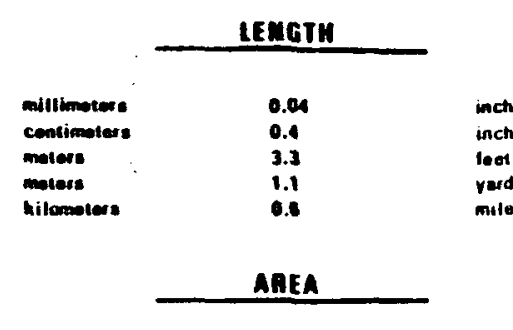

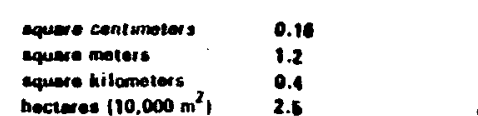

aquere inches

$m_{m}^{m^{2}} m^{2}$

becteres $110.000 \mathrm{~m}^{2}$

cquare verds

ocres

Mass (melntel)

milomernos

0.03

auces

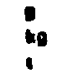

consese (1000

0.035
2.2
1.1

powends

cubic motus:

\section{IEmPEnATURE (exest)}

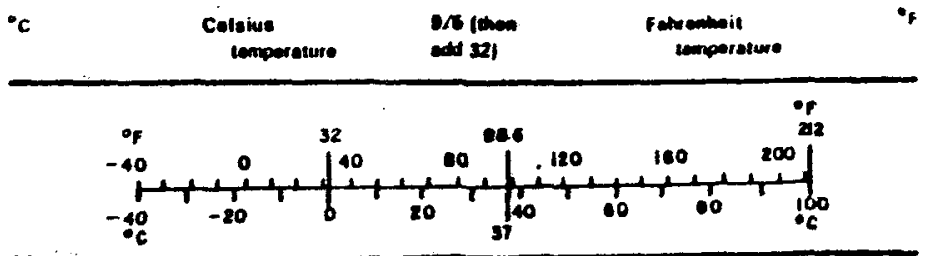


PREFACE

This volume is the final report of a literature review study entitled "The Effects of Fatigue and Alcohol on Highway Safety" performed by Koba Associates, Inc., for the National Highway Traffic Safety Administration of the U.S. Department of Transportation under Contract No. DTNH-22-80-C-07053.

The project staff included Andrea Rauh as Project Manager; Joan Ryder, Ph.D., as Project Director; J. Jepson Wulff, Ph.D., as Senior Technical Consultant; Craig Kinsley and Stacy Malin as Research Assistants; and Ingrid $\mathrm{Hill}$ and Patricia Burnett as Secretaries.

The final report was written by Joan Ryder, stacy Malin and Craig Kinsley with the assistance of the remaining project staff. Additional secretarial support was provided by Ruth Middleton.

The assistance and suggestions of Pamela Anikeeff (Contract Technical Monitor from March, 1980, to January, 1981), Robert Schweitz (Contract Technical Monitor from January to March, 1981) and Tom Ryan, all of the Problem Behavior Research Division of NHTSA, are gratefully acknowledged. In addition, we would like to thank Dr. Dennis Attwood of Transport Canada and Dr. Frederick Hegge of the Division of Neuropsychology at walter Reed Hospital for helpful discussions. 


\section{EXECUTIVE SUMMARY}

This report presents a critical review of recent research, an assessment of the extent of present knowledge, and recommendations for future research to determine the combined effect of fatigue and alcohol on highway safety. The limited data previously available raised the possibility that a number of alcohol-related accidents might be due to fatigue or a combination of alcohol and fatigue. The purpose of this study was to determine what role fatigue might play in alcohol-related accidents. If there is a significant involvement it might be possible to reduce the alcohol-highway safety problem by attacking the problem of fatigue.

The objectives of the literature review were to determine: whether there are valid and reliable measures of fatigue; (2) what relationship exists between the impairing effects of alcohol and fatigue; (3) whether it is possible to estimate the percentage of alcohol-related accidents due to fatigue alone or to a combination of fatigue and alcohol; and (4) whether there are useful remedies to counteract the impairing effect of fatigue.

The present state of our knowledge about the causes, mechanisms and effects of fatigue is still very primitive. Many input factors lead to fatigue. Furthermore, there is no single technique for measuring fatigue that is generally accepted. The input factors which have been found to be most likely to cause impairments in driving performance or performance related to driving are: (1) hours without sleep; (2) time on the task; (3) circadian rhythms; and (4) preceding (stressful) activity. All four factors must be considered in research on fatigue and driving. Three types of measurement techniques have been used in attempts to quanitfy fatigue. These include: (1) physiological changes which accompany fatigue; (2) scales based on subjective ratings of fatigue; and (3) measures of objective performance decrements.

Very little research has examined the combined effect of alcohol and fatigue on driving or driving-related peformance. The research that has been done indicates both synergistic and antagonistic effects, although antagonistic effects predominated. In some cases, high and low alcohol levels produced different interactions. The evidence suggests that low levels of alcohol might reduce the adverse impact of fatigue whereas high levels might increase it. Furthermore, there are large differences in the way in which either alcohol or fatigue or their combination affect different individuals.

The effects of alcohol alone and fatigue alone were also compared to find similarities. Although further investigations must be undertaken to confirm or deny interactions, similarities in performance decrements indicate possible areas in which additive or synergistic interactions may be found. The effects of alcohol alone and fatigue alone on five categories of driving behavior were examined including: 
(1) visual processes, (2) perception and attention, (3) decisionmaking, (4) motor and sensorimotor control, and (5) overall vehicle control.

Neither alcohol nor fatigue has a large effect on the sensory aspects of vision.

A number of similarities were found between alcohol and fatigue effects that are all relevant to a narrowing of attention. These include: (1) decreases in information-processing capacity and rate, (2) reduced ability to process information from the peripheral retina, and (3) less active eye movement patterns. Physiological correlates of arousal decrease and become more variable with fatigue; they decrease with alcohol also, but less consistently. Vigilance (maintaining attention over long periods of time in a monotonous task) is more impaired by fatigue than alcohol.

Complex decision-making tasks are impaired to a greater extent by alcohol than by fatigue, whereas the reverse is true for simple tasks. Motivation and incentive can override some decrements caused by either alcohol or fatigue, and for short periods of time can almost completely override fatigue effects.

Motor and neuromuscular control are more impaired by alcohol than by fatigue. Reaction time increases slightly with alcohol, and becomes more variable with fatigue. Tracking tasks, except very simple ones, are impaired by both alcohol and fatigue.

Vehicle control measures show increased variability as a result of either alcohol or fatigue. Velocity maintenance is generally impaired under alcohol and fatigue, although the changes due to fatigue are not found consistently. Lane position also becomes more variable with both alcohol and fatigue, but the mechanism is somewhat different. Alcohol results in an increase in steering wheel. reversals, while fatigue leads to a decrease in the number of reversals but an increase in the size of steering movements.

Most studies attribute 40 to $55 \%$ of highway fatalities to alcohol intoxication. An assessment of the role of fatigue has been more difficult because fatigue is not defined consistently and there is no reliable way to detect the presence of fatigue in the body as there is with alcohol. Estimates of the involvement of fatigue in fatal accidents ranges from about $2 \%$ to almost 50\%. Although there is evidence suggesting that alcohol and fatigue occur together in many accidents, there is not enough data available to determine what proportion of alcohol-related accidents could be more properly attributed to fatigue. 
Much less effort in the recent past has been directed toward developing fatigue remedies than has gone toward developing and implementing alcohol countermeasures. However, three types of remedies have been suggested including: rest breaks, stimulant drugs, and intermittent stimulation which has a disinhibitory or alerting effect. the most obvious and easily implemented remedy is rest. Caffeine is one stimulant drug that is widely available and useful to aid wakefulness. Another class of stimulant drugs, amphetamines, reduces fatigue-related performance decrements but the possible adverse effects of these drugs limits their usefulness as a remedy. of the possible remedies which use intermittent stimulation, one type is an alerting alarm activated by performance decrements or their correlates such as heart rate variability, number of steering wheel reversals, lane position variability, or some multivariate measure. Performance falling below a set criterion would trigger the alarm.

There is sufficient evidence suggesting that fatigue exacerbates the impairing effects of alcohol on driver performance to warrant further research in this area. One area of research should be directed toward developing a quantifiable measure of fatigue similar to that used to quantify alcohol intoxication (BAC). Subjective fatigue scales appear promising as a research tool but not for enforcement purposes. Physiological measurements of some autonomic nervous system responses (e.g, the pupillary response to light) might be used as a diagnostic tool. A second area of research involves examination of the effects of alcohol on driver performance under conditions of fatigue. It is recommended that research include low, moderate and high alcohol dosages; use primarily closed-course driving tasks or interactive driving simulators; use multivariate measures or test batteries; and control for confounding variables such as personality, motivation, age, sex, mood, and amount of driving experience.

Epidemiological research to determine the extent to which fatigue contributes to alcohol-related accidents is also necessary. The development and testing of an in-vehicle alerting device which is activated by a certain performance decrement is also recommended. Research is necessary to find a single or combined measure that is a valid indicator of driving ability or impairment but is insensitive to the influence of vehicle and roadway factors. 
1.0 INTRODUCTION. . . . . . . . . . . . . . 1

1.1 Background ................. 1

1.2 Study Objectives .............. 2

1.3 Study Method............... 4

2.0 FATIGUE: DEFINITIONS AND MEASURES ....... 6

2.1 Problems Defining and Studying Fatigue . . . 6

2.2 Fatigue Factors . . . . . . . . . . . 7

2.2.1 Neuromuscular Fatigue........ 4

2.2.2 Mental Fatigue. . . . . . . . . 7

2.2.3 Stress Fatigue. . . . . . . . . . 9

2.2.4 Tiredness ............... 10

2.3 Fatigue Measures . . . . . . . . . . . 11

2.3.1 Physiological Measures of Fatigue. . . 12

2.3.2 Objective Measures of Fatigue . . . . 12

2.3.3 Subjective Measures of Fatigue. . . . 12

2.4 Fatigue and Driving. . . . . . . . . . 15

3.0 TYPES OF PERFORMANCE OF PRIMARY INTEREST. . . . 16

3.1 Rationale for the Selection of Types of

Performance of Interest. . . . . . . . 16

3.2 Definitions of Performance Types Selected

for Investigation. . . . . . . . . . 17

3.2.1 Sensory Processes...... . . . 17

3.2.2 Perception and Attention. . . . . . 18

3.2.3 Decision-Making . . . . . . . . 20

3.2.4 Motor and Sensorimotor Control. . . . 21

3.2.5 Overall Vehicle control ...... . 23

3.3 Strengths and Weaknesses of Different

Measurement Settings . . . . . . . 24

4.0 THE EFFECTS OF ALCOHOL ON PERFORMANCE ...... . 26

4.1 Sensory Processes. . . . . . . . . 28

4.2 Perception and Attention . . . . . . . . 30

4.3 Decision-Making. . . . . . . . . . . . 33

4.4 Motor and Sensorimotor Control . . . . . . 35

4.5 Overall Vehicle Control. . . . . . . . 39

5.0 THE EFFECTS OF FATIGUE ON PERFORMANCE . . . . . 42

5.1 Sensory Processes. . . . . . . . . . . 43

5.2 Perception and Attention ..........44 
5.3 Decision-Making. . . . . . . . . . . . 50

5.4 Motor and Sensorimotor. . . . . . . . . 50

5.5 Overall Vehicle Control. . . . . . . . . 52

6.0 A COMPARISON OF ALCOHOL AND FATIGUE EFFECTS UPON

PERFORMANCE . . . . . . . . . . . . . . . 55

6.1 Sensory Processes. . . . . . . . . . . 55

6.2 Perception and Attention . . . . . . . . . 56

6.3 Decision-Making. . . . . . . . . . . . 58

6.4 Motor ans Sensorimotor Control . . . . . . 59

6.5 Overall Vehicle Control. . . . . . . . . . 60

6.6 Summary of Similarities and Differences. . . 61

7.0 ALCOHOL AND FATIGUE INTERACTIONS. . . . . . . . . 62

8.0 ESTIMATES OF THE PROPORTION OF ACCIDENTS CAUSED BY ALCOHOL AND FATIGUE. . . . . . . . . . . 67

8.1 Present Estimated Effects of Alcohol . . . . 67

8.2 Present Estimated Effects of Fatigue . . . . 68

8.3 Estimates of Alcohol and Fatigue Interactions. 70

9.0 POSSIBLE REMEDIES TO COUNTERACT FATIGUE-RELATED

DRIVING IMPAIRMENTS . . . . . . . . . . . . 73 .

10.0 SUMMARY AND CONCLUSIONS . . . . . . . . . . . 78

10.1 Measures of Fatigue Which are Relevant to

Driving . . . . . . . . . . . . . 78

10.2 Relationships Between the Impairing Effects

of Alcohol and Fatigue. . . . . . . . . 81

10.3 Estimates of the Proportion of Alcohol

Related Accidents that can be Attributed to

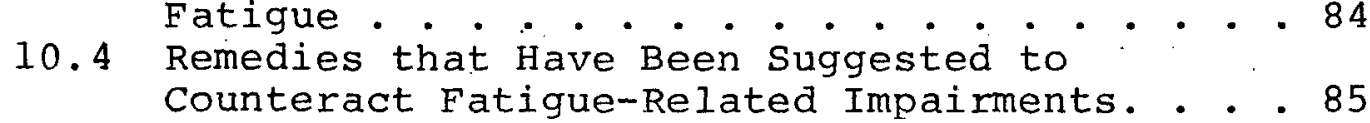

11.0 RECOMMENDATIONS FOR FUTURE RESEARCH . . . . . . . 87

11.1 Development of Fatigue Measures . . . . . 87

11.2 Research to Determine the Relationship

Between the Impairing Effects of Alcohol and

Fatigue . . . . . . . . . . . . . 87

11.3 Epidemiological Research to Determine the

Proportion of Accidents that are Atrributable

to Alcohol, Fatigue, or a Combination . . . 90

11.4 Development and Testing of Remedies to

Counteract the Impairing Effects of Fatigue . 91

12.0 REFERENCES. . . . . . . . . . . . . . . . . . 92 
This Final Report on the "Fffects of Fatigue and Alcohol on Highway Safety" presents a comprehensive review of relevant recent research, an assessment of the extent of present knowledge, and recommendations for the direction of future research to determine the combined effect of fatigue and alcohol on highway safety.

\section{$1.1 \quad$ Background}

It is well established that alcohol is associated with degraded driver performance and highway accidents. The development and implementation of countermeasures to reduce the social cost of alcoholrelated accidents has been a priority of the National Highway Traffic Safety Administration (NHTSA) for many years. Regardless, hundreds of accidents each year continue to be attributed to the intoxicated driver. A possible explanation for this unyielding problem may rest on the inaccuracy of the underlying assumption, namely that these alcohol-related accidents are caused solely by alcohol. Indeed, an in-house NHTSA staff review of $310 \mathrm{multidisciplinary} \mathrm{accident} \mathrm{investi-}$ gation reports revealed that fatigue may serve as a compounding factor in many accidents in which alcohol is a factor. Additional evidence of fatigue's contributing role in highway accidents is also indicated: many accidents attributed to alcohol occur at times when drivers are most likely to be fatigued; and the effects of alcohol and fatigue may parallel each other in many ways.

In spite of indications that fatigue may contribute to alcoholrelated accidents, the manner in which fatigue plays a role remains unclear. It may degrade driver performance alone and/or it may interact with alcohol in a synergistic and/or antagonistic manner to affect performance. The magnitude of the effect of alcohol and fatigue in combination and of fatigue alone on highway accidents also remains unclear. If this impact is substantial, as is suggested, a better understanding of the role of fatigue will be crucial in guiding NHTSA's development of an improved basis from which to direct its future efforts in combating highway safety problems.

As one result of focus on the problem of alcohol as a factor in highway accidents, operational definitions of alcohol have evolved. one of these (BAC--blood alcohol concentration) has been widely used in performance studies. Research on fatigue, on the other hand, has not developed basic well-accepted definitions based on acts of measurement. Because this gives rise to difficulty in addressing tire fatigue issue, development of an adequate measure or definition of fatigue is a primary research objective.

Assuming, nevertheless, one or more acceptable and useful definitions of fatigue, several basic questions need to be answered to place fatigue in useful perspective. First, it is necessary to assess whether or not sufficient experimentation of acceptable quality has 
been performed to indicate an interacting effect of fatigue and alcohol on driver performance. Because past research in this area has been found wanting, future research needs require identification and focus. Secondly, it is important to establish an estimate of the magnitude of the involvement of fatigue in terms of such measures as fatalities per year in order to provide a basis for deciding how to approach the development of countermeasures against driver fatigue. Applied research can then proceed to focus on designing appropriate new remedies that will be more effective in reducing highway accidents.

\section{2 study objectives}

The first part of this study presents a comprehensive review, evaluation and summary of previous research examining the effects of fatigue and alcohol singly and in combination on driver performance. The objective of this review was to determine the following:

The most valid and reliable measures of fatigue.

of particular concern here is the development of operational definition(s) of fatigue that can be employed in various types of research and epidemiological studies. An ultimate goal is to establish a quantifiable measure of fatigue similar to that used to quantify alcohol intoxication (BAC). These initiatives are addressed in Chapter 2 .

The relationship between the impairing effects of alcohol and fatigue.

It is of great interest to assess both the nature and the extent of this relationship. The relationship may be of a synergistic, antagonistic and/or unrelated nature. Because of the limited research findings involving the direct interaction of alcohol and fatigue (see Chapter 7), it is necessary to also examine and compare the performance errors created by alcohol alone and fatigue alone. Is their impairment qualitatively and quantitatively similar or different? Chapter 3 describes the types of performance of interest. The effects of alcohol and fatigue on performance and a comparison of these effects are then discussed in Chapters 4, 5, and 6.

The possibility of estimating the percentage of alcoholrelated accidents due to a combination of fatigue and alcohol and that percentage due primarily to fatigue.

Once the nature of the relationship between alcohol and fatigue has been established, a determination of the scope of its impact on highway accidents is warranted. While 
alcohol has traditionally been held responsible for approximately $50 \%$ of highway fatalities, at least some of this responsibility could more appropriately be attributed to or at least shared with fatigue. Available statistics on the proportion of accidents due to alcohol, fatigue, or both are reviewed in Chapter 8 .

The useful remedies to counteract the impairing effect of fatigue.

To the extent that fatigue plays a pervasive role in highway accidents, it is necessary to design and implement countermeasures that will mitigate this impairing effect. Possible solutions may involve intervention aimed at the driver, the vehicle or the environment. Some remedies have already been implemented as a countermeasure against other impairing agents or conditions and may simply require application to the driver fatigue problem, whereas other remedies still necessitate development and testing. Chapter 9 discusses possible remedies that have been suggested.

An additional objective of this study was to introduce recommendations for future priority research likely to have an impact on driver performance stemming from fatigue alone and fatigue and alcohol in combination. Recommendations for future research are presented in Chapter 11. They address:

The need to develop a more acceptable operational definition of fatigue.

This definition should provide an objective and fairly simple measurement of fatigue and it should ultimately quantify fatigue. It should also facilitate research efforts aimed at understanding the nature of fatigue.

The need for further research that will shed more light on the interaction of alcohol and fatigue and its effect on driver performance.

A very limited amount of research has actually attempted to examine the nature of the interaction of alcohol and fatigue, especially as it relates to driving performance. Thus, an important goal of this report is to outline recommendations for future research that will approach this issue of alcohol/fatigue interactions in an effective and informed manner.

The need for an updated, reliable estimation of the percentage of accidents which are attributable to alcohol or fatigue or to a combination of both these factors.

While both alcohol's and fatigue's effect alone on accidents have been fairly well documented, there is only 
scanty empirical evidence to indicate the magnitude of fatigue's role in alcohol-related accidents (see chapter 8). Thus, there is a need for further fieldwork from which to estimate the actual impact of fatigue on highway accidents.

The need to develop and test feasible solutions to counteract the impairing effect of fatigue.

Although some remedies have been suggested, there is still substantial need for development and testing of possible remedies.

\subsection{Study Method}

An extensive literature review was conducted in order to assess the current state of knowledge on the effects of alcohol and fatigue on driver performance. This search was conducted manually and through the use of computerized data bases focusing on such key areas as alcohol, fatigue, sleep deprivation, driver performance, and drugs and driving.

Because the effect of alcohol has already been the topic of several exhaustive reviews, we have relied largely on these secondary sources rather than attempting to duplicate their efforts. However, we have supplemented these reports by reviewing the alcohol-related research performed over the last five years. Our search of research on fatigue concentrated more extensively on primary sources, especial$1 y$ on research published over the last 10-15 years.

Our primary search included the following sources:

Psychological Abstracts,

MEDLARS at the National Library of Medicine,

National Technical Information service (NTIS),

Smithsonian Institution abstracts (SSIE),

Library of Congress National Reference Service,

Dissertation Abstracts (from 1970 to the present),

Excerpta Medicus (from 1978 to the present),

Social Science Citation Index,

Society of Automotive Engineers (SAE) abstracts,

National Institute on Alcohol Abuse and Alcoholism reference service, 
- NHTSA and Department of Transportation libraries, and

- current contracts.

This search resulted in the examination of over 400 primary sources and reviews of earlier work. The review of published research articles and technical reports was also supplemented through contact with outside researchers who are investigating similar topics. 


\subsection{Problems Defining and Studying Fatigue}

Almost everyone experiences fatigue at some time or another. Yet, almost no one agrees about what it is or how it comes about. Fatigue is commonly used in everyday speech to denote a general set of feelings or sensations including any one or more of the following: tiredness, weariness, muscular aches, task aversion, sleepiness, or boredom. The general usage of the term, as with many terms which refer to psychological states (e.g.. "attitude", "motivation"), is much too vague to be useful in scientific research on fatigue. It does not specify cause, mechanism, or effects, and consequently does not allow any way to investigate relationships among them. What is necessary for research purposes is precise operational (i.e., measurable) definitions of the state of fatigue. That will allow investigation of what input factors cause fatigue, and in what quantity. In addition, it will allow determination of the performance changes that result from any amount of fatigue. Input factors which have been found to cause fatigue are discussed in section 2.2 .

A variety of methods have been used to measure fatigue. However, none of the measurement techniques has yielded sufficiently reliable, replicable results to have led to a generally-accepted fatigue unit or scale. Some measurement techniques that have been used include:

- physiological change measures,
objective performance measures, and
- subjective judgment measures.

These will be discussed in section 2.3 .

Research on fatigue and its effects has proven to be very difficult due in large part to problems defining and measuring it. As early as 1921, researchers realized how difficult it was to define fatigue (Muscio, 1921). Subsequent researchers have examined specific aspects of fatigue (e.g., muscular fatigue, visual fatigue) based on an operational definition of that type of fatigue. However, attempts to relate or compare research findings is very difficult because each investigator has used a different operational definition. Part of the problem may be that "fatigue" is not really a unitary concept, but is instead a number of different psychological and physiological processes which are similar in that they are caused by prolonged activity and result in subsequent deterioration in performance of that activity and/or subjective feelings of wanting to stop the activity. Thus, many factors may cause fatigue, and the fatigue brought about in different ways may or may not be the same phenomenon. 


\subsection{Definitions and Measures of Fatigue Factors}

Although there may be general subjective feelings which are similar for all types of fatigue, performance changes may be different depending on the cause of fatigue. In this section, definitions and measures of various input factors which may cause fatigue will be discussed.

\subsubsection{Neuromuscular Fatigue}

One type of fatigue is that which results from physical exertion. Prolonged muscular work usually leads to reduced ability to continue muscular activity as well as loss of desire to continue. There seem to be two aspects to neuromuscular fatigue (Kinsman and Weiser, 1976). First, the muscles involved become temporarily incapacitated; second, there is an accompanying effect on central mechanisms which results in the general feelings of boredom, etc. After a period of rest, performance usually recovers. After a short rest there may be only partial recovery, in that performance will not return to its peak level or it will fall again more quickly. Longer rests will result in complete recovery.

Much work has been done on neuromuscular fatigue, some aimed at determining performance decrement functions and some aimed at finding the physiological mechanisms of fatigue. Bartley and Chute (1947) summarize much of the early work on neuromuscular fatigue. For a more recent discussion, see the volume by Simonson and Weiser (1976).

Neuromuscular fatigue can be operationally defined as the amount of time a person has engaged in a specific physically demanding activity. That measure will be more accurate if the level of exertion or rate of activity can also be specified. Alternatively, a measure of actual energy expenditure could be used.

Some muscular work is involved in controlling the vehicle, but it is not very vigorous, so driving is unlikely to cause much neuromuscular fatigue. Some muscular stress may develop, however, from maintaining the body in an upright sitting position for a long period of time. But again, that is a minor aspect of fatigue from driving.

\subsubsection{Mental Fatigue}

Mental fatigue, like neuromuscular fatigue, is caused by prolonged activity and results in lowered capacity to continue the activity accompanied by aversive feelings toward continuing. However, in this case the activity is one that need only involve the central nervous system and does not need to include any overt activity. Also, in contrast to neuromuscular fatigue, mental fatigue is not directly related to the amount of energy expended. 
Mental fatigue can be operationally defined as amount of time at a specific mental task. But, it must be noted that not all mental tasks produce mental fatigue at the same rate, nor does the same amount of time at the same type of task always produce the same amount of fatigue. The relationship between the task and amount of fatigue produced is much more complex than that associated with neuromuscular fatigue. Task complexity, interest in the task, motivation or incentive to perform at a certain level or achieve a certain result, and time pressure, among other things, may all influence the magnitude of the effect produced by a certain amount of time at a certain task. Furthermore, there are large individual differences in how "fatiguing" different types of mental activities are or how much rest is necessary for recovery.

It is also not completely clear whether the effects of mental fatigue are specifically attributable to the types of performances that produced them, or whether they generalize to other similar tasks. There seems to be some generalized fatigue from mental activity, but primarily, mental fatigue seems to affect the specific task that produced it, leaving other types of tasks near normal levels. The relative specificity of mental fatigue has led some authors (e.g., Bartlett, 1953; Heimstra, Mast \& Larabee, 1963) to use the term "skill fatigue" to refer to the fatigue produced by specific skilled performances such as driving. Although the authors cited consider skill fatigue to be different from mental fatigue, there is no evidence that fatigue from skilled hehavior is different from fatigue from other mental tasks to any greater extent than would be expected from any two mental tasks or any two skilled tasks.

Welford (1965) distinguishes mental fatigue from monotony or boredom on the basis that the former refers to a state in which the individual is overloaded while the latter refers to one in which there is an underload. In practice, however, this distinction is difficult to make because many of the tasks used to study fatigue are repetitious or characterized by low information content, and thus are likely to become monotonous or boring. Furthermore, since mental fatigue effects are primarily task specific, the same task must be performed over time for fatigue to develop. Therefore, in this report monotony will not be distinguished from other aspects of mental fatigue. Both develop from time on a task, our operational definition of mental fatigue. What is necessary to remember is that different types of tasks often produce different effects.

Some of the types of changes that can come about in mental fatigue include:

sensory or perceptual changes (e.g., loss of either spatial or temporal discriminatory power, changes in criteria of judgement, or decreased sensitivity);

decreases in either speed or accuracy of performance (often either one can be maintained but only at the expense of the other); 
irregularity of performance both in timing and in level (i.e., periods of normal performance interspersed with periods of reduced performance); and

disorganization of performance (i.e., parts of a complex performance unaffected while other parts become impaired; often incidental parts of the task are forgotien; or the ability to coordinate all parts is impaired).

The mental component of driving is much more demanding than the physical actions. Driving requires constant monitoring of the environment, maintaining attention, integrating information, and making decisions about what actions are necessary to maneuver the vehicle safely and on course, all mental processes. Thus, mental fatigue is much more likely to develop from driving then neuromuscular fatigue. Deterioration of any of these component skills must be examined to understand how mental fatigue occurs over time driving. Also, integrated performance of all skills must be examined because component skills might not be impaired in isolation, but only deteriorate when other component skills must be performed simultaneously. Furthermore, performance must be examined under both high and low demand driving situations, since mental fatigue may develop from either overload or underload conditions.

\subsubsection{Stress Fatigue}

Fatigue resulting from emotional or physical stress is distinguished from mental fatigue because specific conditions are necessary for it to arise. In particular, stress fatigue results from either sustained emotional arousal over a period of time, or repeated arousing or stressful incidents. According to Crawford (1961), stress fatigue comes in two stages: a first phase in which increased emotional arousal leads to oversensitive behavior characterized by strong reactions to slight irritations; and a second phase in which there is a reduced intensity of response and a general indifference to minor irritations.

Stress fatigue has been operationally defined in a number of ways, not necessarily comparable. Performance of a task for a certain amount of time can be examined under stressful and non-stressful conditions. Some stressing conditions that have been examined include noise (see Broadbent, 1971, for a summary of the effects of noise), high heat and noise (e.g., Romansky, 1978), and mild electric shock contingent on performance errors (Heimstra, 1970). Accumulated stress from other sources prior to beginning the task of interest can also be used. Time at the preceeding task could serve as an operational definition in this case. Alternatively, experiencing a specific emotionally stressing event could be used.

Driving could be impaired by stress fatigue which develops during driving or which as developed before driving. Crawford (1961) believes that the number of hours behind the wheel may be less important in producing fatigue than the cumulative emotional effects of all 
the stresses and annoyances which are caused by traffic conditions and the behavior of other road users. There is some evidence that stress and distraction augment mental. fatigue in any prolonged task, but that occasional mild stress and novelty help restore efficiency at least temporarily (see Broadbent, 1971, or Kahneman, 1973, for reviews). Therefore, the conditions in which driving takes place must be noted if the performance changes which result over time are to be understood.

Stress fatigue may also have developed from antecedent conditions such as interpersonal problems, job-related problems, arguments with another person, or even the accumulated annoyances of a day's work. Any of these conditions might augment the development of mental fatigue and the consequent performance decrements, or they could create qualitatively different performance changes then other types of fatigue produce.

\section{2 .4 Tiređness}

The term "tiredness" is used here to mean the generalized loss of both physical and mental efficiency which occurs by the passage of time without sleep. Tiredness generally increases with increasing amounts of time without sleep but also follows a 24-hour cycle, (called circadian cycle or diurnal rhythm) of increases and decreases.

The average person sleeps for about $71 / 2$ hours out of each 24-hour day, and is awake for the other approximately $161 / 2$ hours. Also, the sleep period usually coincides roughly with darkness and waking with daylight. Many physiological changes occur in a regular synchronized pattern over each 24-hour period including: body temperature, heart rate, respiration rate, and urinary excretion. The variation is a continuous cycle throughout the 24-hour period, with the peak occurring toward the end of the awake period and the trough near the end of the sleep period. This circadian cycle occurs even without sleep during the normal sleep period and only changes gradually when sleep periods are shifted for extended periods of time (as in changes in time zone or with shift work). Many studies of mental efficiency have found cyclic performance variations that parallel the physiological changes (see a review by Colquhoun, 1971, for further details). In general, performance peaks tend to occur in the late afternoon or evening and the time of least efficiency is about 4 or $5 \mathrm{a} . \mathrm{m}$. However, circadian variations in efficiency are not always found.

In addition to variations in efficiency within normal sleep-wake cycles, efficiency decreases with disruptions in normal sleep. sleep deprivation tends to impair some aspects of psychomotor performance, and extreme cases of sleep deprivation may lead to perceptual disorientation, disturbances in social behavior or psychotic tendencies (see Wilkinson, 1965). Sleep deprivation does not produce uniform effects, even on the same type of performance. In fact, 
motivation can override the detrimental effects of sleep deprivation of up to three days on short tests (less than five minutes)

(wilkinson, 1965). Also, there is variability in how sleep deprivation affects different people, and there is variability due to other concurrent task or circumstantial variables.

Hours without sleep is the most common operational definition of tiredness. Point in the circadian cycle is the second most common. However, other factors are also important including:

- whether there was sufficient or abnormal sleep during the days prior to sleep deprivation,

- whether there was good or poor quality sleep in the preceeding days, even without sleep deprivation,

- point in the circadian cycle of onset of previous sleep (Czeisler, Weitzman, Morre-Ede, Zimmerman \& Knauer, 1980), and

- point in the circadian cycle the time without sleep began.

Of particular importance to the development of fatigue-related driving impairments is whether the driver is rested or tired, and at what point in the circadian cycle driving begins. Changes that occur over time driving may be much greater or develop more quickly for someone who is tired than for someone who is alert. Studies comparing performance with and without sleep deprivation are useful in this regard. Also, studies of performance at different points in the circadian cycle will be useful to examine to gain a better understanding of the times when there are impairments. Since more accidents occur at night than during the day (Department of Transportation Fatal Accident Reporting System Annual Report, 1978, tiredness is an important kind of fatigue to examine in relation to driving.

\subsection{Fatique Measures}

The preceding section discussed operational definitions of fatigue in terms of the factors, or conditions, which usually produce the characteristics described as fatigue. An alternative method of examining fatigue is to measure those characteristics directly.

Thus, fatigue becomes measurable to the extent that its associated characteristics are both defined and measurable. For organ 1 zational purposes, these characteristics or type of measurements can be classified into three categories: physiological, objective, and subjective operational definitions. These measurements are discussed more fully in Chapter 4, especially as they concern specific performance measures. 


\subsubsection{Physiological Measures of Fatigue}

The physiological changes which underlie or cause fatigue are not well-understood. Thus, fatigue cannot be identified by any one single physiological measure. However, some physiological changes often accompany subjective feelings of fatigue, and these changes can provide rough measures of fatigue. Some physiological changes which can be measured include heart rate, galvanic skin response, critical flicker frequency detection, and brain and muscle electrical activity. These measures are discussed more fully in section 3.2.2.

Physiological indicators of fatigue are primarily associated with alertness, arousal, and attention. Most of these indicators are considered to measure changes that occur without any apparent voluntary control. Because of individual differences in these measurements and because of the limited knowledge regarding theit actual significance, these physiological indicators have probably not yet been developed to their full potential (Shinar, Zaidel \& Paarlberg, 1978).

\subsubsection{Objective Measures}

Objective measures of fatigue refer to those measures that are derived from an individual's performance on a task. Differences in performance on the task before and after the introduction of a fatigue factor indicate the "amount" of fatigue. These measures do not take into account any causal factors or the nature of fatigue itself, but rather operationally define fatigue in terms of its outcome. These objective indicators then can only measure those dimensions which are incorporated into the particular task. Various types of performance tasks that have investigated the effects of alcohol and fatigue on driver performance are discussed in Chapter 3 .

\subsubsection{Subjective Measures of Fatigue}

In view of the (aforementioned) difficulties and Iimitations arising from both physiological and objective measures, much effort has been directed toward developing an effective subjective measure of fatigue. Subjective indicators rely on an individual's self-perceptions rather than on any external measuring devices. This is advantageous, at least hypothetically, to the extent that it encourages independence of measurement from both cause of fatigue and specific performance outcomes, facilitating consistency from study to study. However, subjective fatigue can only be measured indirectly through self-reports, on which experimenters are forced to rely. Nonetheless, recent studies have, for the most part, provided fairly optimistic reports concerning the effectiveness of these subjective measures, with effectiveness defined as a good correlation between the subjective measure of fatigue and performance decrements caused by actual fatigue. 
Subjective fatigue is usually measured through scales, questionnaires, and other forms which are marked or reported by the subjects themselves. Subjective fatigue thus lends itself nicely to fairly simple and inexpensive measurements. These types of indicators generally fall into three categories: non-dimensional single point measures, unidimensional rating scales, and multidimensional scales.

Non-dimensional single point measures are the simplest indicators of subjective fatigue, requiring the subject to give just a single verbal report while performing a task. The studies depending on these types of measures have generally reported inconsistent and unreliable findings (Kinsman \& Weiser, 1976). Pierson (1963a), for example, asked subjects performing a reaction time task to indicate when they thought their "responses were becoming slower". While he found a significant relation between this subjective measure of fatique and performance, he later failed to confirm this finding (Pierson, 1963b). As Kinsman and Weiser (1976) conclude, non-dimensional single point measures are generally ineffective in that they view fatigue as. an all-or-none event, rather than recognizing it as a relative phenomenon, affecting performance differently according to its degree or level.

Unidimensional rating scales are generally accepted as a much more reliable measure of fatigue. These scales can be organized into two types: ordinal and interval scales. In a unidimensional ordinal scale, one adjective describing fatigue is selected and ranked in order of magnitude. An example of this is Nunney's (1963) 9-point scale measuring feelings of undifferentiated fatigue ranging from "no fatigue" (1) to "extreme fatigue" (9). Poffenberger (1928) has also devised a 7-point ordinal scale to evaluate feelings of tiredness during prolonged mental work, with the scale ranging from feeling "extremely good" (1) to "extremely tired" (7) and the midpoint (4) indicating "medium" tiredness.

Unidimentional interval scales are similar to ordinal scales except that the intervals between points are equidistant.

Pearson and Byars (1956) have developed a unidimentional interval scale that measures the subjective quality of tiredness. The Feeling-Tone checklist, as this scale is called, has been employed successfully in several studies. The scale consists of an adjective checklist ranked in a continuum with equal intervals between each adjective. The individual checks "better than", "same as", or "worse than" for each of 10 items and the scores indicate his/her placement on the 10-point interval scale. Fatigue, as measured by the Feeling-Tone checklist, was reported by Nelson, Ladan and Carlson (1979) to correlate nicely with task duration. However, alcohol doses showed no effect on self-ratings of fatigue. Kinsman and Weiser (1976) have described the Feeling-Tone checklist as the best subjective scale presently available. 
The stanford Sleepiness Scale (SSS) is another popular unidimensional scale that quantifies subjective changes in sleepiness. It is composed of seven statements that range from "feeling active and vital" to feeling "a little foggy; not at peak" to "almost in reverie; sleep onset soon; lost struggie to remain awake". Hoddes, Zarcone, Smythe, Phillips and Dement (1973) examined the relationship between SSS ratings and performance on mental tasks before and after sleep deprivation. They reported that sss ratings were significantly higher after sleep deprivation than on baseline days and correlated nicely with performance when the mental tasks were long (at least one hour), monotonous and boring. Other unidimensional interval scales rating tiredness have been designed by McNelly (1954) and Bujas, Sremeg \& Vidachek (1965, cited in Simonson and Weiser, 1976).

Without underestimating the value of the unidimensional scale, many researchers have argued that an effective measure of subjective fatigue must take into account more than just quantitative changes in perception. As Kinsman and Weiser (1976) contend, fatigue consists of qualitatively different subjective states that vary according to the task requirements and the individual. "By focusing on only one subjective quality, the essential complexity of the range in subjective qualities is ignored." (p. 374) In this vein, they conceive of fatigue "as a superordinate subjective state into which is funneled discrete subjective symptoms" (p. 386). To serve this view, multidimensional scales have been developed that consider both quantitative and qualitative changes. These scales are usually derived from factor or key cluster analyses that identify groups of symptoms comprising qualitatively different symptom categories. One such multidimensional scale has been designed by Wolf (1967). Using factor analysis, he identified three fatigue categories from a set of 30 adjectives. These categories were labeled: Exhaustion (physically tired, aching muscles, exhausted, easily distracted, no energy and perspiring); Drowsy (mentally sluggish, want to fall asleep, lazy, drowsy, feel sleepy, and tired): and Nervous. (tense, jumpy, keyed up, head tightness, feel dizzy, and irritable).

Multidimensional scales, such as that designed by wolf (1967), have also been developed in Japan, prompted by concern over industrial fatigue. Kogi, Saito and Mitsuhashi (1970) and Saito, Kogi and Kashiwagi (1970) used the "Inventory for the Subjective symptoms of Fatigue", a checklist composed of 30 subjective symptoms divided into three categories, to empirically derive the "Fatigue scale" through factor analysis. The "Fatigue Scale" contains the symptom categories Drowsiness, Difficulty in Concentrating, and Bodily Complaints. While a correlation between subjective fatigue rating and task duration has been shown, specific symptoms and symptom categories have been applicable only with certain work tasks.

Kinsman, Weiser and stamper (1973) have also established a multidimensional scale using cluster analysis. Examining the subjective symptoms associated with working a bicycle ergometer, they identified three categories: Fatigue (describing bodily feeling states 
associated with prolonged exercise); Task Aversion (which appeared to measure a general level of discomfort and disinclination to continue task); and Motivation (describing general drive and vigor). This work is of particular interest because its methodology could be applied to any task although the specific symptoms and symptom categories may vary.

In conclusion, subjective measures, particularly unidimensional and multidimensional scales, appear to be a promising tool for operationally defining fatigue.

\subsection{Fatigue and Driving}

As the preceeding discussion has pointed out, fatigue has proven to be a very intractable object of study. Despite a good deal of research, very little is known about the relationships among subjective feelings of fatigue, objective measures of performance changes, and physiological changes that underlie fatigue. Even when one aspect of fatigue arising from a specific cause is examined, the relationships are imperfect. Consequently, the examination of fatigue and a complex behavior such as driving is bound to be difficult.

Neuromuscular, mental, and stress fatigue all develop from time on a task. The types of fatigue which arise over time driving are primarily mental fatigue and stress fatigue, but very little neuromuscular fatigue. Also, the conditions during driving will affect the type and amount of fatigue and the performance decrement that develops as a result of the amount of time driving. Stress fatigue from conditions prior to driving may also affect driver performance. In addition, tiredness from lack of sleep or from circadian variations in efficiency may impair driving.

Thus, the operational definitions which are relevant to the problem of fatigue and driving are:

- time driving,

- conditions during driving time (monotonous or complex, stressful or not, etc.),

- number of hours without sleep prior to beginning ariving,

- point in the circadian cycle at wich driving begins, and

- the activity proceding driving (stressful or not).

Since no one measure of fatigue has been found to be completely reliable or useful in all situations, all three types of measures should be used in investigations of driving and fatigue including:

- physiological measures,

objective measures of performance decrements, and subjective fatigue rating scales. 


\subsection{TYPES OF PERFORMANCE OF PRIMARY INTEREST}

To determine what is known about the impairing effects of fatigue alone or in combination with alcohol on driver performance, the specific types of performance of interest must be identified and operationally defined. Adequate driving performance can be defined as the safe guidance of a vehicle from one point to another. Following from that definition, the best measure of the effect of alcohol, for example, on performance is the number of accidents caused by drunk compared to sober drivers. However, manipulating alcohol intake and counting the number of accidents that occur is an unacceptable research strategy for two important reasons.

First, legal and moral considerations dictate against examining the behavior of interest directly. Experimental studies must, instead, examine performance in controlled tasks that are assumed to be related to driving, but do not involve the safety risks of actual driving. Of course, the results of these studies are only valuable to the extent that the conditions in which performance is examined are related to actual driving conditions and the measures of performance used are surrogates for actual driving performance. Second, there is no way to determine precisely how alcohol, or any other variable, impaires driver performance when an overall measure such as number of accidents is used. To answer "how" questions, driving must be broken down into component tasks, and decrements in performance on each component examined.

For purposes of this review, five categories of driving behavior will be examined:

- sensory processes,
- - decision-making,
- motor and sensorimotor control, and
- overall vehicle control.

The final category, overall vehicle control, includes measures for which many components of performance contributed to the response.

\subsection{Rationale for the Selection of Types of Performance of Interest}

Much research has been devoted to identifying driving-relevant behavior that is impaired by alcohol. Consequently, our performance typology will include all types of performance that have been studied in relation to alcohol intake and/or $B A C$ level or other mediate 
measurements. The emphasis on alcohol-related performance types will enable a determination of the extent to which fatigue-related performance decrements either mimic or compound alcohol-related decrements.

\subsection{Definition of Performance Types Selected for Investigation}

\subsubsection{Sensory Processes}

Adequate sensory registration of information from the environment and vehicle control displays is necessary before that information can be processed and responded to. Vision is the most important sensory modality for driving, so our review will be restricted to the visual modality. Though it is not clear which types of visual impairments will directly affect driving ability, six areas have been identified as most relevant to driving performance (Perrine, 1973). These include:

- static visual acuity--the ability to discriminate detail in stationary displays (Colson, 1940; Newman \& Fletcher, 1941);

- dynamic visual acuity--the ability to discriminate detail in moving stimuli (Honegger, Kampschulte \& Klein, 1970);

- the extent of the visual field--the area within which objects can be perceived while the eyes are fixated at one point (Colson, 1940; Newman \& Fletcher, 1941);

- adaptation and brightness sensitivity--the ability of the visual system to adjust to high or low illumination conditions (adaptation) and to detect low intensity targets under a given illumination condition (brightness sensitivity) (Lewis, 1972);

glare resistance and recovery--the ability to tolerate an intense light source and still obtain useful information from the surrounding field and the ability to recover from the effects of glare (Mortimer, 1963; Newman \& Fletcher, $1941)$; and

temporal acuity of the visual system--usually measured by the critical flicker fusion $(C F F)$, the slowest rate of flicker at which an intermittent light source is perceived as continuous (Simonson \& Enzer, 1941; Tarter, Jones, Simpson \& Vega, 1971).

Burg (1970), in a comparison of three-year driving records of over 14,000 drivers with visual function, concluded that dynamic visual acuity showed the strongest correlation with driving record and static visual acuity; visual field and glare recovery showed some 
relation. Shinar (1978) reported that dynamic visual acuity, static visual acuity under low levels of illumination, and the ability to perceive small rates of lateral movement were correlated (albeit at a low level) with measures of accident involvement. Driver age and sex affected those correlations; young drivers and males show better acuity than females.

\subsubsection{Perception and Attention}

Rapid processing of a wide variety of stimuli--including the roadway, the car's instruments, other vehicles, traffic signals, pedestrians, etc.,--is necessary for good driving. Not only must the environment be perceived, but the aspects of stimulation which are important for the task must be attended to. A good review of driver inattention has recently been completed (Shinar, Zaidel \& Paarlberg, 1978) and provides the basis for much of this section.

Perceptual and attentional performance is very difficult to measure since it is an internal cognitive process with no directly observable behavioral response. Some physiological changes accompany changes in attention and can be measured, but no one measure has been found to be completely reliable. On the positive side, physiological indicators can be measured continuously in simulators or in on-theroad driving. Furthermore, they are thought not to be under voluntary control, so, to the extent that they are reliable, they can indicate changes over time in response to changing stimulation in realistic conditions. Looking hehavior is often used as a measure of attentional performance and is one of the best available measures.

However, while it is not possible to perceive an object outside the field of view, it is possible to perceive certain aspects of objects that are not fixated, and it is possible that objects that are "looked at" may not be attended to.

Intensive attention. The total amount of stimulation, and hence the amount of attention demanded, is variable--ranging from high at a crowded city intersection to low on a deserted highway. Thus, there is a need for the ability to invest as much attention (effort) as the task demands. This aspect of attention includes the general state of alertness of the individual and the amount of processing capacity or resources that the individual is able to mobilize. Attentional capacity is particularly important to investigate because of its close relation to fatigue, as part of the continuum from alertness to sleep.

There are a number of different measures of attentional capacity including:

heart rate (or pulse rate) and heart rate variability (Romansky, 1978);

respiratory patterns (Dureman \& Boden, 1972); 
muscle electrical activity (electromyogram--EMG) (Lippold, Renfearn \& Vuco, 1960);

skin conductance (electrodermal response, EDR, or galvanic skin response, GSR) (Dureman \& Boden, 1972);

brain electrical activity--patterns of brain wave activity (EEG) (Harris et al., 1972);

pupil size (Lowenstein, 1937);

eyeblink frequency and closure durations (Beideman \& Stern, 1977):

self-assessments of alertness, attentiveness, boredom or fatigue (Romansky, 1978);

driver reports of significant events--i.e., an in-car observer interviews the driver at various points with regard to recent events such as the type of traffic sign just passed (Soliday, 1974; Sanders, 1976); and

subsidiary task performance--a technique which requires the driver to perform an extra task, usually a signal detection or reaction time task, concurrently with the primary driving task. Changes in performance on the subsidiary task are taken as an indication of changes in the surplus attention of the driver and indirectly of changes in the amount of attention the primary task requires (Ellingstad \& Heimstra, 1970; Heimstra, 1970).

Vigilance. In addition to allocating more attention during high demand situations, a driver must be able to maintain adequate attention in low stimulation conditions over a long period of time. This type of performance has been studied extensively in laboratory tasks and many operational settings, such as radar operation and air traffic control, as well as driving. In operational settings, the same behavioral and physiological measures which indicate changes in attentional allocation are used to measure vigilance performance, though the types and amount of stimulation are different. In addition, specialized experimental tasks are used to measure vigilance behavior. For example, subjects watch two small lights on an oscilloscope screen and press a button whenever one moves. Number of errors (failures to acknowledge or perceive the change) serves as the measure of vigilance (Erwin et al., 1978).

Selective attention and recognition. Another aspect of perception and attention which is critical for good driving is the ability to attend to and perceive the stimuli that are important and not be distracted by those that are unimportant. This selective aspect of attention is distinguished from the intensive aspects of attention in that it refers not to the amount of processing resources expended, but to the way the available resources are allocated (Kahneman, 1973). 
The selective aspect of attention has been measured in the following ways:

- eye movement patterns--the pattern of saccades (fast, ballistic eye movements), slower pursuit eye movements, and the locations and durations of fixations--recorded in simulators or instrumented vehicles, or in specialized laboratory tasks (Mackworth \& Morandi, 1967; Noton \& Stark, 1971);

visual search laboratory tasks--tasks in which a subject views a visual display and searches for a specified object. Reaction time to determine the presence or absence of the target is measured (Bertera \& Parsons, 1978):

visually evoked potential--measurement of transient brain electrical activity in the visual cortex during presentation of a stimulus. Electrical activity.usually increases if the stimulus is perceived and attended to (Obitz, Rhodes \& Creel, 1977); and

visual identification or discrimination--laboratory tasks in which a subject has to name a visual stimulus or decide whether two visual stimuli are the same or different.

\subsubsection{Decision-making}

Driving performance also involves making decisions about vehicle control based on interpretations of the information that has been perceived and attended to, knowledge of traffic regulations, and knowledge of the effect any actions will have on vehicle control. Decision processes, like attentional and perceptual processes, are difficult to measure because the decisions themselves are not observable. Furthermore, the physiological changes underlying decision-making are not well understood, so physiological measures do not provide any clues. One way to investigate decision-making is in laboratory situations in which a simple response must be made based on the outcome of a decision, and accuracy or latency to make the decision measured. In simulation or on-the-road driving experiments, more complex responses, such as passing another car, are measures which include a decision component.

Judgments of time, speed, and distance. Accurate judgments of time, speed, and distance are necessary for determining relative velocities of different vehicles, for determining safe following distances, and a host of other critical decisions. Following are some examples of methods for assessing these judgment abilities: 
attempting to maintain an instrumented vehicle at a specified speed without looking at the speedometer. The difference between average vehicle speed and specified speed is a measure of speed judgments (Shinar, McDowell \& Rockwe11, 1974);

Judgments of available passing distance, evidenced either by verbal response or by actual passing behavior (Farber, 1969); and

laboratory tasks in which the time, distance, or speed of movement of small objects are varied and the subject must estimate one or more of them (Gordon \& Mast, 1970).

Judgment of hazard. The ability to judge that a potential danger has arisen and must be responded to is also an important component of driving performance. Though ability to judge hazard is difficult to measure objectively, subjective measures are possible. For example, simulation experiments have been used to present situations with varying risks involved. Judgments of risks are then assessed through use of questionnaires. In on-the-road or simulation studies, subjective counts of misjudgments are often used as dependent measures (Wallgren \& Barry, 1970).

Memory. Decision-making requires adequate memory for recent events. However, memory is usually assessed in laboratory experiments that bear little resemblance to actual driving. Some methods that have been used include digit span tasks or paired associate word memory tasks (Wallgren \& Barry, 1970).

\subsubsection{Motor and Sensorimotor Control}

Sensorimotor control is a very important component of driving performance. It involves the integration of sensory information from the environment and the initiation of appropriate motor actions in response to that continuously changing information. Complex laboratory tasks that involve sensorimotor control over time are good tests of the kinds of skills used in actual driving. Furthermore, motor control alone is a necessary part of driving performance because the driver must be able to maintain an upright sitting position and manipulate the steering wheel and brake and accelerator pedals.

Neuromuscular control. A common indication of intoxication is increased body sway. The Romberg test, in which a person stands as steadily as possible with the eyes closed is frequently used as a measure of standing steadiness. In fact, the Romberg test is often used in police stations as well as research laboratories to measure alcohol effects. According to wallgren and Barry (1970), it is one of 
the most sensitive measures of the detrimental effects of alcohol. Without visual cues, even sober subjects sway somewhat. Other measures of neuromuscular control (e.g., finger-to-finger test, finger-to-nose test) are not as sensitive.

Motor Control. One motor function which is particularly affected by intoxicating doses of alcohol is ocular-motor control. Under the influence of alcohol, when the head is placed on its side, the eyes repeatedly drift slowly upward and then jerk rapidly downward in a sudden compensatory motion called Positional Alcohol Nystagmus (PAN) (Wallgren \& Barry, (1970). This response to the vestibular stimulation caused by the head position is usually suppressed under normal conditions.

Reaction time. A common laboratory measure of sensorimotor performance is reaction time to respond to different types of stimuli. In a simple reaction time task, a subject must respond, usually by pressing a button, to the initiation of some signal. The time from the presentation of the signal to the initiation of the response is an indication of the time necessary for the sensory information to be processed and a motor response initiated. Other, more complex, reaction time tasks require the subject to make one of two or more possible responses depending on the type of signal presented. For example, the task might be to press the right key if a green light is presented and the left one if a red light is presented. Complex, or choice, reaction time tasks measure decision processes as well as sensorimotor processes. Thus, it is possible to measure the increase in reaction time as the difficulty of the decision increases. For example, the difference between simple and choice reaction time is a rough indication of the effect of increasing decision-making demands. Since those drivers for whom decision times are shorter are more likely to be able to respond to a critical situation in adequate time, reaction time measures are useful in examining driver performance.

Tracking. Tracking tasks also involve sensorimotor coordination. While tracking tasks vary considerably in complexity, they basically require a person to monitor a visual display and respond continuously. A prototypical experimental situation is the following. A subject views two vertical lines on a screen. One line moves at random, the second line moves when the subject moves a controlling stick to the right or left. The subject attempts to keep the two lines coincident, and the amount of time off-target is the dependent measure (Dott \& McKelvy, 1977). In a somewhat simpler task, an index just has to be maintained at a predetermined position. The former task is often referred to as pursuit tracking and the latter, compensatory tracking (Murrell, 1965).

Since tracking tasks can involve a fairly complex combination of sensory processing and motor responding over time, they are very good laboratory tasks to investigate the skills used in driving. The continuous nature of the tasks is also a key factor in their usefulness, since that more closely simulates actual driving. 


\subsubsection{Overall Vehicle Control}

The fifth category of driver performance involves the execution of actual motor responses which control the vehicle's course. These are overt responses which can be measured easily in an instrumented vehicle or simulator. The use of controls in a driving or simulated driving situation varies as a function of the complexity of the situation and the skill of the driver (Attwood, Williams, \& Madil, 1980; Perrine \& Huntley, 1971). Monitoring control use, therefore, provides a good way to investigate changes in driving performance due to alcohol and/or fatigue. Furthermore, these measures are taken as part of an actual driving situation so their face validity is high. However, responses in a complex driving situation reflect perceptual and decision processes as well as response execution, so they may not be used to isolate the component driving skills that are impaired.

Lateral vehicle control is accomplished by moving a steering wheel. Steering behavior includes large steering movements necessary to make turns and small back-and-forth corrections to keep the car on course even on a straight road. An increase in the rate of corrections, known as steering reverslas, often is an indication of driving difficulty. Two measures of steering behavior (Dott \& McKelvey, 1977; Platt \& Fedderson, 1964) are:

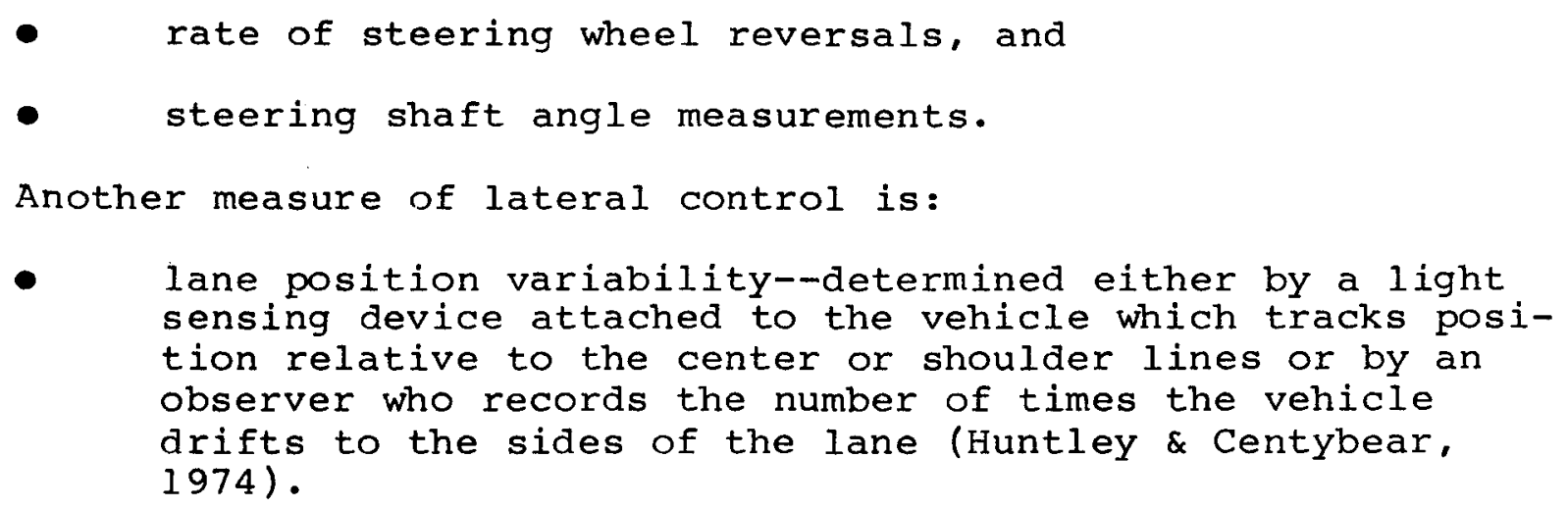
tion relative to the center or shoulder lines or by an observer who records the number of times the vehicle drifts to the sides of the lane (Huntley \& Centybear, 1974).

Longitudinal control requires the foot movements of pressing the accelerator and brake pedals and shifting the right foot from one pedal to the other. Measures of longitudinal control (Attwood et al., 1980; Huntley \& Centybear, 1974) include:

- accelerator applications,

- brake applications,

- accelerator noise--the standard deviation of acceleration, and

speed variability. 
There are also some measures of response execution which include both lateral and longitudinal control. These include:

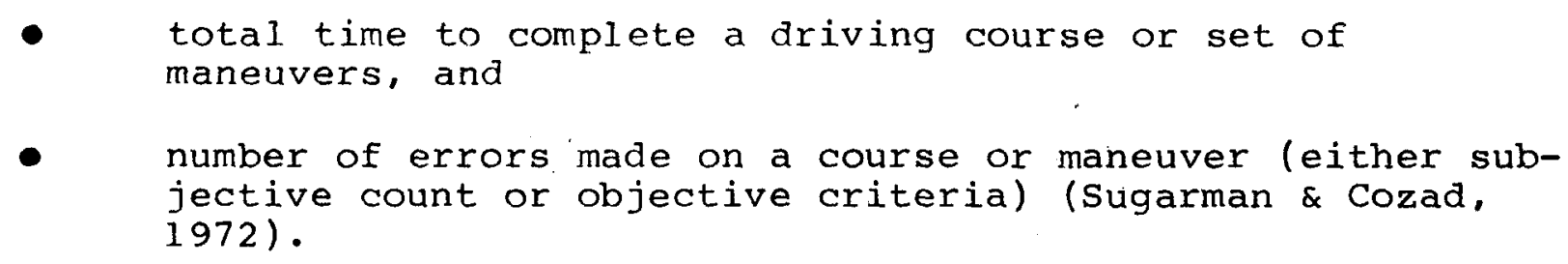

3.3 Strengths and Weaknesses of Different Measurement Settings

A short description here of the general types of experimental procedures and measures and the strengths and weaknesses of each will be useful in evaluating the different ways to measure each performance component. The methodologies for examining ariver performance include:

- controlled on-the-road studies,
- - whole-task driving simulator studies,
- component-task simulator studies, and
laboratory studies of tasks which are related to driver
- performance.

There are advantages and disadvantages to each methodology which must be considered in their evaluation. On-the-road performance is directly generalizable to actual driving since task complexity and response requirements are the same as in actual driving. However, it lacks control over environmental variables (i.e., movement of other cars or pedestrians) that might influence performance. Laboratory studies, in contrast, maintain greater control of possible confounding environmental variables allowing their results to be attributed to the effect of the manipulated variables with greater confidence. However, what laboratory studies gain in control, they lose in generalizability. Laboratory tasks are usually complex situations requiring responses over a shorter time period, and thus, are less directly related to actual driving performance. Studies using sophisticated simulators which create the illusion of driving are, of course, closer approximations to real ariving behavior than studies of component skills.

Despite their drawbacks, laboratory studies are particularly important for examining performance impairments due to alcohol or fatigue because of the safety risks involved in on-the-road studies. Furthermore, all studies, whether on-the-road or laboratory, must take 
into account the fact that subjects are aware that their performance is being monitored. Thus, behavior in any experimental situation is probably more indicative of maximal performance in the task rather than usual performance. Finding performance decrements in experimental studies, when motivation is high and task demands may be less strenuous than in real driving, is clearly generalizable to real driving. However, caution must be exercised in generalizing from findings of no performance decrement. The point is that most experimental studies yield conservative estimates of performance decrements due to various impairments. Furthermore, the simpler the task, in - comparison to actual driving, the more conservative the estimate of performance decrement. 
During the last fifty years, much research has been directed toward investigating the physiological and performance effects of alcohol. Since the magnitude of the involvement of alcohol in causing traffic fatalities is well known, much of that research is now directed toward investigating the role of alcohol on ariver performance. This chapter will review studies that have examined the effect of alcohol on driver performance and on performance related to driving as a basis for comparing the effects of fatigue on those performances. Since the amount of literature dealing with alcohol and performance is massive, this report will be based mostly on reviews except for very recent work. Furthermore, we will give only a brief summary of the effects that have been found and refer back to specific articles for the details of the experiments.

One of the most comprehensive reviews of the effects of alcohol is a two-volume book published by wallgren and Barry (1970). A second valuable source is a compilation published as a result of a vermont symposium on alcohol, drugs, and driving which was held in 1972. A special issue of the Journal of Safety Research in 1973 summarizes the material covered at that symposium. More recent literature is also cited in a review by Moskowitz and Austin (1979) on the effects of alcohol on human skills performance.

Alcohol ingestion produces physiological and behavioral changes most commonly refered to as intoxication. Although the exact mechanism by which intoxication is produced is not known well, most researchers believe that the behavioral changes due to alcohol ingestion are mediated by the effects of alcohol on the central nervous system. In particular, the reticular activating system, which plays a central role in the control of sleep, wakefulness, attention and arousal, as well as regulating cortical activity, is thought to be the major site of alcohol effects in the nervous system. Also, many physiological processes of the body are altered by alcohol and disturbances in psychomotor functioning as well as intellectual processes sometimes occur.

Alcohol is usually characterized as having a depressant effect on the body; however, there is some evidence that high doses have a depressant action while low doses have a stimulating action. One explanation of this "bi-phasic effect" is that alcohol is always a depressant and that its apparent stimulating effect at low concentrations is due to a depressing of some inhibitory mechanism (Wallgren \& Barry, 1970). Whatever the explanation, however, the existence of this bi-phasic effect makes it imperative that performance be examined at various doses. 
There is no simple relationship between alcohol consumption and behavioral changes. The amount of alcohol necessary to produce a certain behavioral change is affected by the age and sex of the person, the person's history of alcohol use, the contents of the stomach, body-weight, and general physical health. However, despite this variability, the concentration of alcohol in the blood provides a useful measure with which to relate alcohol consumption and human behavior. Blood alcohol concentration (BAC) is a measure of the amount of alcohol in a person's bloodstream stated as a percentage of weight per unit volume. BAC is a well defined easily measurable parameter primarily affected by alcohol intake. In most states in the U.S., the legal level of intoxication is $0.10 \%$. This amount of alcohol is enough to produce clear behavioral changes in the majority of the population. For purposes of this review we will refer to low, medium, and high BAC levels which will indicate <0.05\%, $0.05 \%-0.08 \%$, and $>0.08 \%$, respectively, since behavioral changes seem to be different at these three levels.

A number of problems exist in trying to integrate the findings of various researchers and to present general conclusions. These problems, noted by Levine and his associates (Levine, Kramer \& Levine, 1975), include the following:

- the design of studies differs widely according to whether subjects are used as their own control, or whether there is a separate control group;

- subject factors (e.g., sex, weight, age, or type of drinker) which are known to influence alcohol-related behavior often are not taken into account;

the method of determining the dosage and the method of administering alcohol often differ widely between experiments;

- the types of alcoholic beverages used often differ, a problem because different types of alcoholic beverages have different absorption rates;

- the blood alcohol concentration level often varies;

the time variables, which may markedly influence performance, are often uncontrolled--these include the time allowed to consume the alcohol, the time of day alcohol is ingested, and the time allowed to pass from the beginning of drinking to the beginning of testing.

Furthermore, many published reports failed to give adequate information on the above variables, failed to test a wide range of alcohol doses, and used widely varying performance measures. Especially early research (including that reviewed by Jellinek and McFarland, 1940) 
used small numbers of subjects, inadequate controls, and no statistical tests to determine the significance of the alcohol effects

reported. Despite these problems, this chapter will attempt to summarize the effects of alcohol on the performance measures detailed in the previous chapter.

\subsection{Sensory Processes}

static visual acuity. A number of studies have shown that the impairment in static visual acuity rises commensurately with increases in BAC. For example, at low BAC's no impairment of static visual acuity has been shown (Brecher, Hartman, \& Leonard, 1955; Colson, 1940; Marquis, Kelly, Miller, Gerard, \& Rapoport, 1957; Mortimer, 1963). Impairment is found for some subjects at moderate blood alcohol concentrations (Brecher et al. 1955; Colson, 1940; Mortimer, 1963; Newman \& Fletcher, 1941; Seehafer, Huffman, \& Kinzie, 1968; Verriest \& Laplasse, 1965). At BAC levels above $0.10 \%$, a high proportion of subjects show impairment $(85 \%)$ (Newman \& Fletcher, 1941). Wallgren \& Barry (1970) conclude from their review of the literature on the actions of alcohol that visual acuity is "rather insensitive to the effects of alcohol" (page 288). Thus, static visual acuity seems to suffer only with very high levels of alcohol.

Dynamic visual acuity. Dynamic visual acuity, the ability to distinguish closely separated moving objects, seems to be impaired at much lower BAC levels than static visual acuity. Honegger,

Kampschulte, and $\mathrm{Kl}$ ine (1970) report impairment in some subjects at BAC's as low as $0.02 \%$ and report impairment for all subjects at BAC's over $0.10 \%$. Dynamic visual acuity requires not only retinal processes, but also ocular-motor coordination involved in accomodation. Levett and Karras (1977) found that moderate and high alcohol doses brought about a 10-30\% slowing of accomodation responses, which may contribute to the decrease in dynamic visual acuity. Furthermore, it requires perception of objects that are moving over time, so it is more likely to be related to driving performance than is static visual acuity. Good dynamic visual acuity was found to be positively related to good driving records for either accident or conviction rate records (Burg, 1970).

Visual field. Four early studies of the effects of alcohol on the extent of the horizontal visual field have found that even relatively high doses do not cause any appreciable reduction in terms of sensory sensitivity (Colson, 1940; King, 1943; Newman \& Fletcher, 1941; Peters, 1942). However, recent studies examining peripheral vision in situations where the requirement for information processing is more complex, such as a divided attention task, have reported extensive impairment by alcohol (Buikhuisen \& Jongman, 1972; Hamilton \& Copeman, 1970; Huntley, 1970, Moskowitz \& Sharma, 1974; Von Wright \& Mikkonen, 1970). This narrowing of the field of view has been referred to as "tunnel vision". It has been noted (Moskowitz, 1973; Moskowitz \& Austin, 1979) that when peripheral vision is examined with 
no other demands on the suhject, alcohol produces no impairment. it is only when the subject is simultaneously performing some other visual task, usually at the central fixation, that peripheral vision is impaired. Thus, the visual field reductions which have been observed appear to be the result of attentional limitations rather than sensory impairment.

Adaptation and brightness sensitivity. Two early studies (Lange \& Specht, 1915, cited in Jellinek \& McFarland, 1940; Yudkin, 1941) found a slight improvement in dark adaptation and brightness sensitivity at low levels of alcohol. This beneficial effect of small doses of alcohol has not been replicated in more recent studies (Blomberg \& Wassen, 1959; Giardini, 1948). Other studies (Lewis, 1972; Lewis \& Sarlanis, 1970) found little or no deterioration at low and moderate BAC's, but showed significant impairment at BAC's greater than $0.08 \%$. Since the majority of fatal highway crashes occur at night under conditions of low illumination, the impairment of this visual function is important to investigate.

Glare resistance and recovery. Perrine (1973) reports that only three studies have specifically addressed this question (Mortimer, 1963; Newman \& Fletcher, 1941; Verriest \& Laplasse, 1965). These three experiments used slightly different tasks and came up with results that were inconsistent with each other. However, in summary they point to a lack of evidence to indicate that glare resistance is appreciably decreased by BAC's up to $0.08 \%$. Even at higher concentrations the majority of subjects do not show a decrease in glare resistance. A recent study by Sekular \& McArthur (1977) found a decrease in recovery from glare at high alcohol levels.

Temporal acuity. The critical flicker fusion threshold (CFF) is used as an index of the temporal resolution, or temporal acuity, of the visual system, as well as as an indicator of central nervous system function and condition. Most of the experiments in the literature are in general agreement that high doses of alcohol (greater than 0.08\%) lower CFF threshold; however, the effect is generally quite small (See Wallgren \& Barry, 1970). The effects of low BAC levels, however, are less consistent; some investigators report decreases in CFF with BAC's as low as $0.02 \%$ whereas other investigators report no change at the lower BAC's. One study (Lewis, Dustman \& Beck, 1969) even reported a slight elevation in CFF at the lowest blood levels tested (0.03\%) and no effect at higher levels (up to 0.09\%). Regardless of whether the impairment in temporal acuity has any significant effect on driving skill, the available data suggest that the temporal acuity of the visual system is slightly impaired by high BAC's. One possible explanation for the conflicting results found by Lewis et al. (1969) may be that practiced subjects were used in that experiment, and practice is known to mitigate the effects of alcohol (Tarter, Jones, Simpson, \& Vega, 1971) and to change the criteria for psychophysical judgments. 


\subsection{Perception and Attention}

Intensive attention and vigilance. The ability to maintain adequate attention is closely correlated with the state of arousal of an organism. Several physiological measures which are thought to be correlated with the state of arousal have been investigated following alcohol ingestion. The primary measure that has been investigated is changes in the electroencephalogram (EEG). A large number of studies summarized in Wallgren \& Barry (1970) show that moderate and large doses of alcohol decrease the frequency and increase the amplitude of cortical activity, showing predominantly depressant effects. At low doses of alcohol slight stimulant effects have been found by a few investigators. It should be noted, however, that individual variation in EEG patterns is great and EEG changes often show poor correlation with behavioral signs of intoxication. Furthermore, changes are usually more marked with rapidly than with slowly increasing blood alcohol content, and the correlations with behavioral signs of intoxication are lower when blood alcohol is falling. Thus, dosage and time between ingestion and recording are important in determining the relationship between EEG and alcohol ingestion.

Changes in evoked potential after direct sensory stimulation have also been found following administration of ethanol. Khristozov and Atsev (1960) found that the suppression of alpha activity which accompanies visual or auditory stimulation was diminished by intoxicating doses of ethanol. Obitz, Rhodes, and Creel (1977) administered high doses of alcohol to subjects and found a reduction in visually evoked potentials and an increase in reaction time. The introduction of an incentive (monitary reward), however, attenuated the effects of alcohol on both the evoked potential and reaction time. Moderate doses of alcohol were similarly found to reduce the auditory evoked responses (Gross, Begleiter, Tobin \& Kissen, 1966).

The ability to monitor low quantities of information over long periods of time (vigilance) is very slightly lowered with moderate levels of alcohol (Moskowitz \& Austin, 1979). The relative insensitivity of vigilance to moderate levels of alcohol was observed in several studies (Colquhoun, 1962; Docter, Naitol, \& Smith, 1966; Moskowitz \& DePry, 1968; Moskowitz \& Sharma, 1974; Talland, Mendelson, \& Ryack, 1964). Moskowitz (1979) does report that there are some cases in which vigilance is impaired under the influence of alcohol, but he suggests that "The context of the experiments suggests the decrement in vigilance might have been due to an interaction of alcohol with other factors such as fatigue."

Heart rate has also been used as a physiological measure of arousal under the influence of alcohol. Wallgren and Barry (1970), after reviewing several studies, have reported that moderate levels of alcohol have been shown consistently to produce a slight, short-lived increase in heart rate. This increase may be enhanced with physical exertion. (See Wallgren \& Barry, 1970, for a more extensive review). 
Also used as an indicator of intensive attention is the arlramic skin response (GSR). Alcohol, even at low doses (e.q., 0.0548 BAC) has been shown to reduce GSR during mental tasks (Ijienert \& Traxel, 1959 ) and in response to loud sounds (Carpenter, 1957; Greenberg \& Carpenter, 1957). This latter finding provides evidence that alcohol may have a fear-reducing effect. However, alcohol has sometimes been found to increase GSR, for example, while performing a difficult perceptual task (Coopersmith, 1964). Thus, the effect of alcohol on GSR may depend upon the conditions of the experiment.

Selective attention and recognition. Tasks in which various amounts of information from the environment must be processed, selected, and recognized appear to be sensitive to even very low levels of alcohol. These effects may be due to a decrease in either the rate or amount of information that may be processed at any one time. In a number of different information processing tasks, Moskowitz and his associates found decrements in the rate of information processing under alcohol. They found an increase in the psychological refractory period (Moskowitz \& Burns, 1971), an increase in latency to name visually presented objects (Moskowitz \& Roth, 1971), an increase in the time necessary to recognize four letters presented tachistoscopically under conditions of backward masking (Moskowitz \& Murray, 1976), and an increase in the rate of switching attention between ears when letters were presented dichotically (Moskowitz \& Keller, 1979). In a recent review article (Moskowitz \& Austin, 1979), it was proposed that alcohol's detrimental effect upon the rate of information processing may be at least partially responsible for the accompanying decrements in divided attention and skilled performance tasks.

Divided attention tasks have been found to be particularly sensitive to the effects of alcohol (Moskowitz \& Austin! 1979). Performance on divided attention tasks has been impaired at blood alcohol levels below 0.05\% (Moskowitz \& Sharma, 1974; Moskowitz\& Roth, 1971) and even at levels as low as 0.015 to $0.017 \%$ (Hamilton \& Copeman, 1970; Moskowitz \& Shea, unpublished paper cited in Moskowitz, 1973). When alcohol levels reach $0.08 \%$ subjects have almost universally demonstrated impairment in performance on divided attention tasks. A series of studies by Gruner and colleagues (1955, 1963, 1964 ) on the effects of alcohol on the performance of two simultaneous tasks found evidence for considerable impairment under moderate levels of alcohol. Other studies have also found impairment when two simultaneous tasks were required but no impairment when either one of the two tasks was performed alone. With moderate alcohol doses, Moskowitz \& DePry (1968) found no impairment on either an auditory signal detection task or an auditory short-term memory task. However, when the two tasks had to be performed simultaneously there was a sharp performance decrement on both tasks. Similarly, Buikhuisen and Jongman (1970) found differences between an alcohol group and a placebo group only when the two groups had to monitor several scenes simultaneously and no alcohol effect when only one visual aspect had to be monitored in a scene. 
Allen, Rex, McRuer and DiMarco (1975) reported that when attention was divided between a continuous and a discrete task while operating a driving simulator under the influence of alcohol, some performance decrements were enhanced. In particular, the demands of the discrete detection recognition task disrupted performance on the continuous steering task, while, the steering task did not impair performance on the discrete task.

The effects of time sharing were also evident in investigations of the extent of the visual field. In the previous section, we discussed how the extent of the field was decreased only when subjects were concurrently observing something in their central fixation field. Moskowitz and sharma (1974) conclude that this impairment is not an impairment in peripheral vision, but instead has to do with the detrimental effect of dividing attention.

Measuring eye movements during visual search or target detection tasks is another way to obtain information about the distribution of attention and the way in which objects are recognized. One important aspect of eye movement behavior is fixation duration. Several studies have reported an increase in fixation duration under alcohol intoxication (Beideman \& Stern, 1976; Belt, 1969; Buikhuisen \& Jongman, 1976; Schroeder, Ewing, \& Allen, 1974). These longer fixation times are most likely due to the additional time needed for processing information received during each fixation. A decrease in the frequency of fixations has also been found (Beideman \& Stern, 1976; Moskowitz, Zeidman \& Sharma 1976), indicating reduced visual search efficiency. Another aspect of eye movement behavior that has been investigated is scanning patterns (indicting the area which receives the most attention). While some studies reported an increase in attention to central areas (Beideman \& Stern, 1976; Belt, 1969; Buikhuisen \& Jongman, 1972), other studies failed to find this effect (Moskowitz et al., 1976; Mortimer \& Sturgis, 1972). These inconsistencies have been attributed to the different conditions presented in each study with regard to the task demanded of the subject, and consequently, the strategies adopted by the subjects toward those tasks. Furthermore, the scanning patterns of intoxicated drivers are less active and less affected by the presence of other objects or cars on the road (Belt, 1969; Moskowitz et al., 1976).

Bertera (1978) found that alcohol may have a more permanent detrimental effect upon visual search performance. He found that detoxified alcoholics (regardless of duration of alcoholism) showed impaired visual search speed. Also, long-term alcoholics, in particular, displayed a subtle visual search performance deficit, possibly associated with right hemisphere disfunction that may be apparent only when the task is more demanding.

In a more recent study, Moskowitz and Zeidman (1979) confirmed their previous findings that alcohol impairs both the processing of information and the proper distribution of attention to different aspects of the driving task. On a driving simulator, under the 
influence of alcohol, subjects showed increased dwell time and visual search durations. They also showed impaired performance in steering and speed control as well as in the time required to read route signs. Unusually long fixations or gazes were also noted, suggesting that attention is momentarily either ceased or extremely attenuated. Familiarity with the route displayed on the driving simulator mitigated the effects of alcohol to a small but significant degree by decreasing the amount of information needed to be processed.

\subsection{Decision Making}

Judgements of time, speed, and distance. Wallgren and Barry (1970) review several studies of the perceptual judgement of the passage of time. They found that with moderate amounts of alcohol time seems to pass more quickly, and consequently, responses designating an estimated time interval are longer (Joerger, 1960; Sterzinger, 1935; Wolff, Hardy \& Goode11, 1941). Rutschmann \& Rubinstein (1966) also found that alcohol increases the variability of the perception of time intervals.

Impairments in distance or depth perception have also been found as a result of alcohol (See Wallgren \& Barry, 1970). Newman and Fletcher (1941) found significant impairments in the judgment of distance under the influence of alcohol, but only minor impairments in a measure of depth perception. Starck (1953) found that high doses of alcohol (BAC's well above $0.10 \%$ ) increased the time necessary to complete a task which involves depth perception. Chardon, Boiteau and Bogaert (1959) report impairment of depth perception at low BAC's for $15 \%$ of their subjects. A possible reason for the impairment of distance or depth perception is the fact that alcohol impairs binocular coordination (Brecher, Hartman \& Leonard, 1955; Wist, Hughes \& Forney, 1967). Distance judgments, which are based partly on sensory feedback for muscles which control binocular convergence, may consequently be impaired. In a highway driving task, Mortimer and Sturgis (1979) found that BAC's of $0.085 \%$ did not significantly affect judgments about the speed and minimum time necessary to safely pass another car.

Judgment of hazard. Since alcohol seems to lower inhibitions and raise one's level of aggressiveness, it seems to follow that an individual would be more willing to take risks. However, research in this area has yielded inconsistent results. In an investigation using professional drivers, Cohen, Dearnaley \& Hansel (1958) found that subjects with BAC's of $0.04 \%$ made attempts to drive a bus through a gap which was to narrow for the vehicle. However, for driving in traffic this result did not hold up. In two studies using low to moderate alcohol levels (Ehlers, 1966; Tegar, Katkin \& Pruit, 1969), subjects under the influence of alcohol were more likely to choose a risky alternative in hypothetical situations.

Another fairly common finding concerning judgment is that many people under the influence of alcohol feel that their performance is normal or even better than normal at times when objective measures 
show definite performance impairments. Cutter (1969) found that subjects given very low doses of alcohol (about $0.015 \%$ BAC) overestimated their choices of success in a hypothetical game despite the

experimenter's specification of the level of luck. Lubin (1977) found that subjects consistently underestimated their impairment on a reaction time task at both moderate and high levels.

In a simulator study with signal light, curve negotiation and obstacle avoidance tasks, Allen, Schwart, Hogge \& Stein (1978) found that risk-taking behavior, such as going more frequently on a red light, increased with alcohol intoxication (BAC>0.10\%). However, their analysis showed that the changes were not due to the drivers accepting higher risks. Instead, the changes were due to distorted perception of risk and degraded psychomotor performance.

Memory. Many studies have found that alcohol impairs memory, especially short-term memory. Wallgren and Barry (1970), reviewing experiments on the effects of alcohol on memory, point out that decrements might he attributed to impairment of the original learning, impairment in retention, or impairments in attentiveness. Whatever the mechanism for the impairment in memory, most decision-making processes require some sort of memory and, thus, may be affected as a consequence of memory impairment. Ryback (1971) has reviewed the effects of alcohol on short- and long-term memory. In general, large detrimental effects of alcohol on short term memory have been found. Several other studies reviewed in Wallgren and Barry (e.g., Hutchinson, Tuchtie, Gray \& Steinberg, 1964; and Nash, 1962) found that the deleterious effect of alcohol on short-term memory for digits or words was greater when more complex material was used or when the task was more complicated (i.e., the need to reorganize the material or recite the digits backwards). Ryback concluded from his review that long-term memory was somewhat disrupted, but not nearly as much as the disruption in short-term memory. Jones (1973) found that although immediate, short-term, and long-term memory are all influenced by alcohol ingestion, short-term memory seems to be particularly influenced. Most of the effects of alcohol on long-term memory are found for retention of information learned while intoxicated and tested subsequently while sober. This type of effect, often called statedependent learning, really is not relevant to performance impairments while driving under the influence of alcohol.

Problem solving. Wallgren and Barry (1970) review a number of studies on problem solving tasks including arithmetic calculations, digit symbol substitutions, and spatial relation tests. The results for arithmetic calculations are variable, but generally show that alcohol impairs accuracy rather than speed and is greater when the task is made more difficult. Digit symbol tests and spatial relation tests both seem to be impaired by alcohol, but most of the studies reviewed used fairly high doses and both of these tests include a considerable degree of sensorimotor performance. Since sensorimotor coordination is often impaired under the influence of alcohol, it is 
likely that performance on these tasks will also be impaired. Tests requiring the manipulation of verbal concepts have generally shown rather small but consistent detrimental effects of alcohol.

Detrimental effects of alcohol in solving more complex problems have been found in many experiments. However, a few experimenters have reported that low alcohol doses improved certain types of problem solving (Carpenter, Moore, Snyder \& Lisansky, 1961). Some recent studies (Sidell \& Pless, 1971; Reisby \& Theilgaard, 1969) have indicated that intellectual functions are much more resistant to the detrimental effects of alcohol than are sensorimotor functions. Furthermore, many experimenters have found that difficult tasks are affected by alcohol to a greater extent than more simple tasks (Landauer \& Milner, 1971; Lewis, 1973; Hurst \& Bagley, 1972).

\subsection{Motor and Sensorimotor control}

Neuromuscular control. The Romberg test of standing steadiness has been found extremely sensitive to the impairing effects of alcohol (Kelley, Myrsten, Neri \& Rydberg, 1970; Wallgren and Barry, 1970). In laboratory experiments, substantial increases in swaying were found at BAC's ranging from $0.075 \%$ to $0.15 \%$ (Franks, Hensley, starmer \& Teo, 1976; Fregly, Bergstedt \& Graybiel, 1967; Goldberg, 1954; Idelstrom \& Cadenius, 1968) and increases were sometimes found at alcohol levels as low as 0.038 (Hebbelinck, 1963). However, many drunken drivers did not display swaying when given the Romberg test even at BAC's as high as $0.10-0.20 \%$ (Laves, 1955; Prag, 1953). Perrine (1973) suggests that when faced with the threat of being convicted for DWI, many individuals are able to override the effects of alcohol upon body sway or other aspects of neuromuscular control. Perrine also questions the validity of any neuromuscular measure as an indication of impairment in actual driving performance because of variability among individuals even with the same blood alcohol levels.

Motor control. Disturbances in ocular-motor control have been found as a function of alcohol. Placement of the head in a lateral position induces nystagmus eye movements, called Positional Alcohol Nystagmus (PAN), under the influence of moderate to high alcohol levels (Aschan, Bergstedt, Goldberg \& Laurel1, 1956; Fregley et al., 1967; Walter, 1954). PAN does not occur in the absence of alcohol or if the vestibular system is not intact. Under normal conditions ocular-motor control is strong enough to suppress the nystagmus eye movements which in impaired conditions occur in response to vestibular stimulation created by lateral head position (Wallgren and Barry, 1970).

According to Aschan et al. (1956), PAN first occurs at fairly low BAC's (0.038\%) beginning about a half-hour after alcohol consumption and lasting three hours on average. A reversal in direction of PAN begins about one or one and a half hours after the first phase ends and lasts five to ten hours depending on the amount of alcohol consumed. 
Reaction time. Many experiments have examined the effect of alcohol on reaction time in response to a visual or auditory stimulus. Early experiments reviewed in Jellinek and McFarland (1940) and Carpenter (1962) generally found an increase in reaction time with moderate alcohol doses, although the effect was usually slight. Alcohol doses (from $0.12-2.1 \mathrm{~g}-\mathrm{kg}$ ) yielding BAC's of $0.012-0.128$ caused reaction time to increase from 5 - $17 \%$ (Blum, Stern \& Melville, 1964; Boyd, Morken \& Hodge, 1962; Buffard, 1959; Carlson, Kleitman, Muehlberger, McLean, Guliickson \& Carlson, 1934; Muller, Tarpey, Georgi, Mirone \& Rouke, 1964). Carlson et al. (1934) and Joyce, Edgecombe, Kennard, Weatherall and Woods (1959) were the only researchers who reported a decrease in reaction time: (RT), and both the decrease $(2-5 \%)$ and the amount of alcohol ingested were slight $(0.3 \mathrm{~g}-\mathrm{kg}-0.7 \mathrm{~g}-\mathrm{kg})$. Wallgren and Barry (1970, p. 299) conclude that while alcohol increases reaction time, it is necessary to reach a BAC of at least $0.07 \%$ "to obtain consisent effects". When BAC's of 0.08 - $0.10 \%$ are obtained, RT is usually lengthened by no more than 10\%. Consistently large impairments in performance require a BAC of at least $0.15-0.20 \%$.

Jellinek and McFarland (1940) suggested that complex tasks might be more sensitive to intoxication than simple tasks. Carpenter's (1962) finding that alcohol lengthened choice RT but not simple RT confirms this suggestion. Similarily, three other studies found that alcohol increased choice reaction time more than simple reaction time (Bahnsen and Vedel-Petersen, 1934; Carlson et al., 1934; Rauschke, 1954). With more complex tasks, Gruner (1955, 1959), Gruner and Ptasnik (1953) and Zirkle (1959) all reported that the impairing effect of alcohol could become even more pronounced. These results imply that the decision component of RT as well as the motor component are affected by alcohol.

Alcohol had a debilitating effect upon RT when subjects were required to respond to stimuli presented at the same time as another task. (Rafaelsen, Bech \& Rafaelsen, 1973; Rafaelsen, Christup \& Bech, 1973; Sugarman, Cozad, \& Zavola, 1973). Alcohol also increases errors in choice RT tasks (Martin. LeBreton \& Roche, 1957) and produced greater variability in times, particularly by increasing the incidence of long RT's (Cass \& Frederick, 1961; Martin et al., 1957; Teichner, 1954).

Both Heacock and Wikle (1974) and Sutton and Burns (1971) found evidence of the degrading effects of alcohol on RT, but they did not interpret this as a direct causal relationship. Instead, they suggested that alcohol may interact with other variables, which may then lead to increased rection time. Heacock and Burns, for example, suggested that subjects may misperceive when to read; sutton and Burns (1971) cited sex differences as a confounding variable. Obitz, Rhodes and Creel (1977) supported this notion in their report that the amount of motivation affects reaction time. They claimed that low levels of motivation interacted with moderate levels of alcohol to produce an increase in reaction time due to "inattention" rather than to a motor impairment. 
The failure of some experments to consider and control for the speed-accuracy tradeoff in reaction time performance tests has been held partly responsible for the inconsistent findings on the role of alcohol. Shillito, King and Cameron (1974) and Jennings, Woods and Lawrence (1976) have found that reaction time differs according to whether or not accuracy is stressed as a performance criterion. Thus, low levels of alcohol (i.e. BAC's of .033 - .05\%) may be associated with no apparent decrement in speed when accuracy is not stressed. However, when accuracy is stressed, RT may still be maintained, but only at the cost of an impairment in accuracy, especially at higher doses of alcohol (Shillito, King, \& Cameron, 1974; Jennings, Woods \& Lawrence, 1976)

In sensorimotor tests of response speed which require sustained response to more complex stimuli than the reaction time test, alcohol slowed performance speed (i.e., Jellinek \& McFarland, 1940; Bohne, Luffe \& Trautman, 1957; Gruner, 1955, 1959), although the extent of this reduction varied from one study to the next. An increase in errors was consistently found in some performance tests as a result of alcohol intoxication (i.e., Wilkinson \& Colquhoun, 1968; Lawton \& Cahn, 1963; Nash, 1962; Goldberg, 1943). Performance accuracy on rapid motor coordination tasks was also adversely affected by alcohol (Idestrom \& Cadenius, 1968; Joyce et al., 1959). However, those speed tests stressing motor dexterity rather than accuracy in choice selection were less susceptible to the effect of alcohol (e.g., Lawton \& Cahn, 1963).

Tracking. Jellinek and McFarland's (1940) review of several studies on tracking indicated that they all reported impaired tracking performance as a result of moderate levels of alcohol. More recently, Drew, Colquhoun and Long (1958) found that increasing doses of alcohol from $0.01 \%$ to $0.08 \% \mathrm{BAC}$ led to progressive increases in tracking errors from a $1 \%$ decrement at the lower level to a $16 \%$ decrement at the higher level. Other investigators have reported that tracking errors increased by 9-148 with BAC levels of $0.05 \%$ (Dott \& McKelvy, 1977; Forney, Hughes \& Greatbach, 1964; Hughes \& Forney, 1964; Hughes, Forney \& Richards, 1965). Newman, Fletcher and Abramson (1942) have shown that while individual differences in tracking performance are found following the ingestion of alcohol, the general trend is that increasing doses of alcohol cause similar decreases in tracking accuracy.

Tracking tests can be made to resemble real driving situations more closely by the use of a steering wheel and a simulated road display, as well as by requiring the driver to control speed in addition to direction. In tracking tests resembling the driving situation, tracking performance impairment was exacerbated under the influence of alcohol. Bjerver and Goldberg (1950) showed a decrease both in speed and in accuracy in driving maneuvers. Steering accuracy was sizeably impaired by blood alcohol levels of 0.02 - $0.08 \%$ (Asknes, 1954). Wallgren and Barry (1970) conclude that while tracking is generally impaired by alcohol, this impairment is enhanced when attention is divided among multiple tasks. 
While the effects of alcohol on tracking performance vary from one study to the next, consistent findings may emerge when tracking is divided into compensatory and pursuit types of tasks. "In compensatory tracking, an index has to be maintained at a predetermined position whereas pursuit tracking requires a control index to be kept in alignment with an index that is also moving" (Moskowitz, 1973, p. 188). Thus, pursuit tracking requires simultaneous monitoring of two or more information sources, whereas compensatory tracking only requires monitoring of one source.

Studies examining the effect of alcohol on compensatory tracking tests did not reveal any decrements in performance when attention was focused solely on this task (Pearson, 1968; Collins, Schroeder, Gilson \& Guedry, 1971; Chiles \& Jennings, 1969). However, when other variables were introduced, such as the stress of hypoxia (Pearson, 1968), angular acceleration (Collins, et al., 1971) and subsidiary tasks (Chiles \& Jennings, 1969), compensatory tracking performance became more susceptible to alcohol intoxication. Alcohol may also have a deleterious effect upon compensatory tracking when it is administered in larger doses. Newman (1949), for example, found decrements in performance at a BAC level of $0.182 \%$. Moreover, Moskowitz (1973) suggests that those studies where compensatory tracking remained immune to the effects of alcohol did not employ very complex tracking tasks. In support of this notion, Reid, Hansteen and Miller (1972) reported that alcohol impaired peformance on certain aspects of complex tracking tasks. Reid and Ibraham (1975) also found degraded tracking performance as a result of alcohol intake.

Low levels of alcohol have been more consistently associated with decrements in pursuit tracking performance whether attention was focused solely on tracking or was shared with a subsidiary task. Hughes and Forney (1964), Asknes (1954), Loomis and West (1958) and Mortimer (1963) all found impairment in pursuit tracking at BAC levels as low as 0.05 or $0.06 \%$. However, in some studies low BAC levels did not affect performance (Gibbs, 1968; Hamilton \& Copeman, (1970; Von Wright \& Mikkonen, 1970). One study failed to find a decrement in pursuit tracking even at a BAC of $0.10 \%$ (Gibbs, 1966). Evans, Martz, Rodda, Kiplinger, and Forney (1974) examined pursuit tracking peformance under the influence of different levels of alcohol. The majority of subjects did not display decrements in performance until they attained a BAC of $0.077 \%$, while all subjects showed impairment at $0.089 \%$.

Two other studies (Colquhoun, 1962; Talland, Mendelson \& Ryack, 1964) found that alcohol did not impair simple tracking performance, even at BAC's above 0.10\%. However, like Moskowitz and Wallgren and Barry, they also point out that more complex tracking tasks and tasks requiring divided attention or timesharing do become sensitive to smaller doses of alcohol. Billings and Wicks' (1972) finding that a BAC of 0.048 impaired performance on a timesharing tasks substantiates this opinion. Compensatory tracking performance is affected only when it is performed concurrently with another task. In 
contrast, pursuit tracking is impaired with BAC's as low as $0.05 \%$. Thus, it is concluded (Moskowitz, 1973; Moskowitz and Austin, 1979) that the decrements in tracking tasks are due to increased information processing demands rather than to an impairment in neuromuscular control.

\subsection{Overall Vehicle Control}

Alcohol's effect on overall vehicle control has been investigated through a broad range of research techniques. While most studies do not employ flawless experimental designs, the quality of these studies varies considerably. Most experiements have examined performance on either a driving simulator or on actual closed-course driving tasks, with technical instrumentation ranging in sophistication from study to study. The performance measures have also varied greatly, but can be readily divided into two groups. The first category includes measurements collected from direct human observation and judgment, while the second relies on instrumentation (Huntley, 1973). Regardless of these differences, almost all the studies have reported performance decrements in driving under the influence of alcohol.

A common performance measure, especially in the earlier alcohol studies, concerns driving accuracy in terms of easily observable decrements such as number of pylons upset or elapsed time necessary to complete a task. Alcohol intoxication was found to increase the number of stanchions hit on closed-course driving tasks in several studies (Coldwell, Pennex, Smith, Lucas, Rodgers \& Darroch, 1958; Taylor \& Stevens, 1965; McLellan, 1969; Seehafer, Huffman \& Kinzie, 1968 ) but serious weaknesses in the experimental designs of many of these studies (i.e., Coldwell et al., 1958; Taylor \& Steven, 1905; Chastain, 1961; MCLellan, 1969) render them suggestive at best. Both Lovibond and Bird (1970) and McLellan (1969) reported that the number of upset pylons increased with inceasing BAC levels, but Lovibond and Bird found that this sensitivity to alcohol varied for competitive and non-competitive drivers. Non-competitive drivers showed noticeable impairment at a BAC of $0.05 \%$ while competitive drivers did not display any apparent impairment until they attained a BAC of $0.08 \%$. Low levels of alcohol also impaired driving accuracy in emergency situations, with subjects reacting by upsetting more pylons and colliding into a man-shaped obstacle (Laurel1, 1977). Huntley and Perrine (1971) agreed that alcohol may degrade driving accuracy in terms of the number of upset pylons, but they found that this impairment was conditional. Specifically, when accuracy was emphasized in the instructions to the subjects, alcohol intoxication was no longer associated with a decrement in driving accuracy.

Alcohol also was shown to have a detrimental effect upon the time necessary to complete a task. Bjerver and Goldberg (1950), in one of the earliest studies to look at the effects of alcohol on actual driving, required subjects to complete perfectly a series of maneuvers. They found that a mean BAC of $0.045 \%$ was associated with increased time at the task. Coldwell et al. (1958) found that the 
time to complete low-speed driving tasks was impaired at $0.075 \%$ for half their subjects while Taylor and stevens (1965) indicated that all their subjects showed slower and less coordinated driving at a BAC of over $0.10 \%$

Longitudinal vehicle control has been used as a more sensitive indicator of alcohol intoxication, with particular emphasis placed on variables associated with stopping, car following and velocity maintenance performance.

Stopping performance involves the ability to brake smoothly and at the right time and place. Attwood, William and Madil (1980) reported that subjects under the influence of moderate levels of alcohol were unable to stop a car smoothly in response to a red light, instead braking sooner and more quickly than normal subjects. Damkot, Toussie, Akley, Geller and Whitmore (1977) similarly found that high BAC levels $(0.08-0.149 \%)$ caused drivers to stop less smoothly as they approached a road block on an actual road. These findings concur with that of Huntley and Kirk (1972) who reported that subjects reduced their braking time by $25 \%$ while under the influence of alcohol. Braking distance was also found to increase under both low and moderate levels of alcohol (Lovibond \& Bird, 1970; Laurel1, 1970; Crancer, Dillie, Wallace \& Haykin, 1969; Rafaelson et al., 1973). Driving simulator studies also confirmed that braking performance is impaired by alcohol. Huntley and Perrine (1971) have shown that braking impairments under alcohol are irreversible even when accuracy is stressed as an important performance measurement. On the other hand, Attwood et al., (1980) found that performance on a closed-course stopping task was rarely associated with intoxication even at higher doses $(e . g ., 0.079 \%$ BAC).

Velocity-maintenance performance, while varying according to the BAC level obtained, is also generally impaired under the influence of alcohol. Most studies reported an increase in accelerator reversals at moderate to high doses (Attwood et al., 1980; Mortimer \& Sturgis, 1979; Huntley \& Centybear, 1974; Belt, 1969) even when accuracy was stressed in the experiment (Huntley \& Perrine, 1971). However, Attwood et al. (1980) reported that velocity-maintenance performance was fairly resistant to low BAC levels (0.033\%). Alcohol also did not significantly affect judgments regarding the speed and minimum time necessary to safely pass another car (Mortimer \& sturgis, 1979) or the ability to reach different velocities without speedometer feedback (Belt, 1969; Mortimer \& Sturgis, 1979). The specific nature of alcohol's effect on speed also seems unclear. Jansen (1960) found that high levels of alcohol accelerate speed whereas coldwell et al. (1958) reported a slight decrease in speed when subjects were given lower doses. Abele (1958) found that intoxicated professional drivers reduced their average speed while increasing variations in speed, whereas Damkot et al. (1977) and Huber (1955) found that alcohol increased driving speed. 
Car-following performance, which requires control of both stopping and velocity-maintenance appears extremely sensitive to alcohol. Attwood et al. (1980) reported that car-following was impaired even at very low doses (0.033\% BAC). Belt (1969), however, indicated that subjects under the influence of both low and moderate levels of alcohol displayed narrowed visual search patterns while driving on the open road but not while following another car.

Steering behavior has also been used to detect driving impairment under the influence of alcohol, but with inconsistent results. Seehafer et al. (1968) found that moderate levels of alcohol (BAC $0.05 \%$ - 0.07\%) significantly increased the number of steering reversals, while Huntley, Kirk, and Perrine (1972) reported an increase in coarse steering reversals at higher BAC levels $(0.085 \%-0.116 \%)$. However, alcohol showed no effect on steering reversals in Huntley and Perrine's (1971) study and Martin (1971) found that low alcohol levels (BAC's of $0.045 \%$ - $0.051 \%$ ) actually produced better operator efficiency than did no alcohol, as measured by the number of fine steering reversals. In this study, the high alcohol group (BAC of $0.09 \%)$ performed relatively worse than did both the low alcohol and the normal groups. On gross steering reversals, alcohol showed no effect at all. However, the driving tests ran for only 15 minutes, thus weakening the findings of this study.

Steering reversals appear to be more susceptible to alcohol when considered in terms of lane position. Steering errors, defined as lane deviations, were increased both on a driving simulator (Crancer et al., 1969) and in actual driving (Abele, 1958; Allen et al., 1975; Huber, 1955; Huntley \& Perrine, 1971; Sugarman et al., 1973). Mortimer and Sturgis (1979) did not concur, however. They found that lateral position steering errors were not significantly affected by moderately high (0.085\% BAC) levels of alcohol on highway driving both during the day and at night.

While it generally appears that alcohol will impair ariving performance, the exact nature of this impairment remains unclear. Most performance measures show impairment at moderate to high levels, but this cannot always be expected. Other influencing factors must also be taken into account, such as driving experience, drinking experience, personality and the nature of the driving tasks (Huntley, 1973). 


\subsection{THE EFFECTS OF FATIGUE ON PERFORMANCE}

As the last chapter demonstrated, many factors affect the relationship between alcohol consumption and driving performance. In addition to the problem of determining which physiolgical and behavioral measures are good indicators of driving impairments, many endogenous and exogenous factors alter the relationship between the amount of alcohol consumed and these performance measures. The use of blood alcohol concentration (BAC) readings as measurements of an intervening state have provided some clarification however.

A critical examination of fatigue's effect on performance is even more complex than that of alcohol. First, as was pointed out in Chapter 2, the literature does not offer a well-accepted definition of fatigue. In addition, fatigue, however defined, may result from any of a number of input factors (e.g., sleep deprivation, time driving, activity performed in the immediate past), each of which may produce similar or different performance changes. Furthermore, there is no well-defined intervening state corresponding to "fatigue" in general that can be reliably measured.

With alcohol, there is one manipulable input factor of overriding importance--alcohol intake; with fatigue there are many. With alcohol there is a mediate measure--BAC--which is a function of alcohol intake and which reduces the variability in alcoholperformance relationships; there is no analogous mediate measure of fatigue. In fact, the use of fatigue as an intervening state may add uncertainty as compared with the determination of straightforward relationships between a fatigue input factor and performance measures.

Our review of the fatigue-performance literature will be organized in the same manner as the previous chapter on alcohol and performance. For each study cited, the input factor contributing to fatigue will be noted. When different fatigue factors lead to the same type of performance change, tentative general conclusions can be drawn. However, when different input factors yield different results, the reason for the conflict cannot be determined. The difference might mean that fatigue does not produce a consistent effect. Alternatively, it might mean that different input factors consistently produce different effects.

Although fatigue has long been recognized as a potential problem in driving, its effect on ariving and driving-related performance has not been investigated as thoroughly as that of alcohol. one line of research has been concerned with determining reasonable maximum limits on the time commercial vehicle drivers should be permitted to drive continuously (e.g., Harris, Mackie, et al., 1972; Jones, Flinn \& Hammond, 1941). Other research on fatigue has been concerned with industrial productivity and shift work (e.g., Colquhoun, Folkhard, Knauth \& Rutenfranz, 1975) and with military probleins (e.g., Pfeiffer, Siegal, Taylor \& Shuler, 1979). 
The examination of driving performance using simulators began in the 1950's and has continued to the present. During this time, the technology, and consequently the experimental sophistication, has improved dramatically. Thus recent work with driving simulators is, in general, much more reliable than earlier work. Furthermore, recent studies often show differences that were not evident in earlier studies.

A good review of the early work on fatigue and performance can be found in the Department of Transportation report "A Study of the Relationships among Fatigue, Hours of Service, and Safety of operations of Truck and Bus Drivers" (Harris et al., 1972). A more recent review is contained in a report on fatigue and performance to the Pennsylvania Transportation Institute (Olsen and Post, 1979). Fatigue and measures of attention are discussed at length in Shinar, Zaidel, and Paarlberg (1978).

\subsection{Sensory Processes}

Much less work has been done investigating visual processes as a function of fatigue than as a function of alcohol. No experiments on either adaptation and brightness sensitivity or glare resistance and recovery were found. A few investigators have examined visual acuity and visual field extent as a function of some type of fatigue. The most work has been done with temporal acuity. In fact, critical flicker fusion has often been used as an index of fatigue.

One important point to note with regard to fatigue and vision is that the level of fatigue is often higher at night than during the day, and vision is more restricted at night simply because of darkness. Thus, vision may often be impaired when fatigue is high, although the sensory decrement does not result from increased fatigue.

Static and dynamic visual acuity. Berger and Mahneke (1954) found that visual acuity decreased by 18-32\% over an hour period during which continuous judgments were made. After a five-minute rest period, substantial recovery (to 70-82\% of the pre-work level) had occurred. Suzumura $(1962,1963$, cited in Joscelyn et al., 1979) found that the fatigue associated with aircraft piloting, physical exertion, and sleep deprivation influenced dynamic visual acuity but not static visual acuity.

Visual field. Some research has indicated that the peripheral retina is more impaired than the focal retina by stress, fatigue, or visual noise (Mackworth, 1971; Weltman \& Egstrom, 1966). Thus, after a prolonged period of driving, it may be that less information is obtained from the peripheral retina than under rested conditions. However, as was the case with alcohol, this narrowing of the field of vision is more likely the result of attentional limitations rather than sensory impairment. No direct tests of the sensitivity of different parts of the visual field under various levels of fatigue have been conducted. 
Temporal acuity. Critical flicker fusion (CFF) was proposed as a test of subjective fatigue (Simonson \& Enzer, 1941) because it is an index of the general state of excitability of the visual system and probably of the central nervous system. Bartley and Simonson (1976) review a number of studies of CFF and conclude that CFF decreases with strenuous mental, physical or visual work, but is more variable when measured intermittently during occupational work. The variability observed during work may have resulted from changing working conditions because CFF declines more consistently over long monotonous conditions (Grandjean, 1970), with self-reports of increasing fatigue (Grandjean et al., 1977), and with driving time (Ohkubo; 1976; Yajima, Ikeda, Oshima, \& Sugi, 1976). Yajima et al. (1976) found that CFF varied as a function of time of day with the low point about $4 \mathrm{a} . \mathrm{m}$. and the high point about noon. They also found a decrease in CFF due to time-on-task ( 10 hours) superimposed on the diurnal rhythm.

Other sensory effects. Some studies of sleep deprivation contain reports that perceptual anomalies are experienced by some subjects (Kleitman, 1939; Mosley, 1953). The reports include visual misperceptions, temporal disorientation, and hypnagogic hallucination of non-existant objects. Usually the episodes are transitory and interspersed with periods of more normal perceptual functioning.

Prolonged driving may also lead to visual fatigue. However, visual fatigue more often results from prolonged viewing of very close objects or displays, rather than more distant ones as in driving. See Schmidtke (1965) or Smith (1979) for further discussion of visual fatigue and its effects on sensory processing.

\subsection{Perception and Attention}

Intensive attention and vigilance. The linkage of fatigue and inattention has led many researchers to attempt to determine which physiological measures can be used as reliable indices of fatigue. Furthermore, physiological changes such as heart rate, respiration rate, galvanic skin response (GSR), electroencephalogram (EEG), electroculogram (EOG), or electromyogram (EMG) can be recorded continuously during a long driving task so that changes can be investigated over time or correlated with changing stimulation and demands.

Heart rate typically decreases over time during prolonged driving (Boadle, 1976; Fagerstram \& Lisper, 1977; Harris, Mackie et al., 1972; Lisper, Laurell \& Stening, 1973; Mackie \& Harris, 1977; Mackie \& O'Hanlon, 1977; O'Hanlon \& Kelly, 1977; Riemersma et al., 1977; Sugarman \& Cozad, 1972) and in a simulator (Dureman \& Boden, 1972). Yajima et al. (1976), in contrast, did not find any significant change in heart rate over a 7-10 hour period on the open road. No consistent decreases in heart rate over time have been found during laboratory vigilance tasks (O'Hanlon \& Beatty, 1977; Thackray, Bailey \& Touchstone, 1977 ) or during one hour of pursuit tracking (Douglas \& 
Johns, 1973). Heart rate sometimes increases during traffic events of significance, such as intersections (Rutley \& Mace, 1972) and fluctuates as a function of both physical and mental effort (clayton, 1971).

Heart rate generally increases in variability for the relaxed, unalert individual. Many investigators have found increased heart rate variability over long drives under boring monotonous conditions (Harris, Mackie et a1., 1972; Mackie \& O'Hanlon, 1977; O'Hanlon, 1971; O'Hanlon \& Kel1y, 1974; Riemersma et al., 1977b; Sugarman \& Cozad, 1974). O'Hanlon (1971) further noted that heart rate variability decreased after alerting events such as running over lane markers, after rest pauses, and during difficult driving. Riemersma et al.. (1977a) found that heart rate was higher and less variable on difficult in-town routes than on easier rural routes.

Heart rate variability increased with time on a vigilance task in some cases (O'Hanlon \& Beatty, 1977) but not in others (O'Hanlon \& Kelly, 1977; Thackray et al., 1977).

Riemersma et al. (1977b) argue that changes in heart rate represent a change from high arousal to normal arousal rather than from normal to low, primarily because decreases are greatest at the beginning of a trip. Futhermore, pre-test heart rates were lower for subjects expecting a full night of driving than for control subjects expecting to sleep during the night. This indicates that heart rate changes could be attributed to adaptation to a task and changes in arousal level but not of fatigue due to time-on-task.

Romansky (1978) examined performance on choice reaction time tasks over a three-hour period under normal or stressful conditions (moderate heat and noise). Heart rate variability showed a sawtooth pattern superimposed on an increase over time, was higher overall in the stress condition, and fluctuated considerably toward the end of the stress condition. Thus, not only did time on the task increase variability, but so did other stress-inducing factors.

Most of the research investigating changes over time has not controlled for diurnal rhythms. Riemersma et al. (1977b) began testing all subjects at $10 \mathrm{p} . \mathrm{m}$.; however, they recognized that some of the changes could be due to diurnal rhythm rather than time driving. Harris and Mackie (1977) found that many physiological indicators of arousal, including heart rate, show diurnal cycles with the lowest levels occurring between about 2:00 a.m. and 7:00 a.m.

EEG patterns provide a gross indication of overall electrical activity of the brain. Higher frequency waves (beta and gamma) are found in alert individuals and during perception of a stimulus. Slower alpha waves are common when an individual is physically relaxed but awake and not in a stimulating environment. Even lower frequency waves (theta and delta) appear during deep sleep, but also occasionally in an awake individual. 
Long-term driving under tedious conditions has in many studies shown that alpha activity increases over time (Caille \& Bassano, 1977; O'Hanlon \& Kelly, 1974; Mackie \& O'Hanlon, 1977; Sugarman \& Cozad, 1972; Yajima et al., 1976). The same pattern was found in a simulator study (Sussman \& Morris, 1970). Some investigators have even found transient delta or theta activity (Caille \& Bassano, 1977; O'Hanlon \& Kelly, 1974).

Another indication of changes in attention is pupil size (Beatty \& Wagner, 1978; Hess \& Polt, 1964). Direct correspondence between pupil size and information-processing demands on a second-by-second basis have been demonstrated independent of external light conditions and physical aspects of the stimulus. Yoss and his associates (Yoss, 1969; Yoss, Moyer \& Hollenhorst, 1970) have found that pupil size fluctuations can be used to indicate changes in alertness, and they have used this technique to diagnose sleepy drivers.

Change in skin conductance (GSR) is often viewed as one of the best physiological indicators of momentary changes in stress or arousal. However, it has not proven a consistent measure of change over time. Yajima et al: (1976) found that GSR decreased gradually over 12 hours of driving from a higher than normal level at the beginning. No significant trends were found in either of two simulator studies (Dureman \& Boden, 1972; Sussman \& Morris, 1970). In O'Hanlon \& Kelly's (1974) on-the-road study, GSR increased slightly over 3-5 hours of driving even though other measures (e.g., EEG and heart rate) indicated decreasing arousal.

other physiological measures have been examined including respiratory rate and volume (Lisper et al., 1973), EMG (Sussman \& Morris, 1970), and blood pressure (Yajima et al., 1976). Since those measures have not been examined in alcohol studies, they will not be discussed here. Another potential measure of fatigue is the change in the pupillary reflex to light (Lowenstein, 1937; Lowenstein \& Lowenfeld, 1952). They found that prolonged mental work led to slowing of the pupillary reflex movement as well as increased variability.

As this short review should have made apparent, physiological measures are not always consistent indicators of attention. These measures are somewhat sensitive to changes in arousal, but are also affected by situational changes and motivation. Some measures such as GSR are primarily sensitive to momentary changes while others such as EEG frequencies are more indicative of longer-term attentional demands, while heart rate measures are affected by both short- and long-term demands. Thus, no one measure is completely reliable or useful in all situations. Sugarman and Cozad (1972) suggest a combination of measures using multiple regression as an alertness index. such an approach could potentially improve reliability over any individual measure. They propose a combination of alpha and theta EEG rhythms with mean heart rate because they found all three to be correlated with some performance changes. O'Hanlon (1971), after reviewing 
evidence for many measures, felt that heart rate variability was the best measure. In summary, it should be noted that the state of the art with regard to physiological measures of fatigue is still rather primitive. Some trends are found, but there are no reliable indices at the present even using combined measures.

Many of the studies of physiological correlates of attention are useful in pointing out trends, hut contain sufficient problems to warrant concern against drawing strong conclusions based on these studies. The number of subjects tested is often small (e.g., o'Hanlon, 1971), and while changes over time are shown graphically, no statistical tests are done for either trend or difference from controls or pretest conditions (O'Hanlon, 1971; Yajima et al., 1976).

Another measure of attentional capacity that has frequently been used is performance on a subsidiary task while performing another primary task. The logic behind this measure is that as the primary task becomes more demanding or the subject's total effort or attention declines, performance on the subsidiary task will suffer. The research with subsidiary tasks during actual driving or driving simulators, however, have not yielded consistent results.

Riemersma et al. (1977a, b) examined performance changes on two subsidiary tasks--reporting mileage and reaction time (RT) to respond to changes in the color of a light on the dashboard--during continuous nighttime driving. Both tasks demonstrated an accelerating decrement over the night, as would be expected if attention waned during that time. Missed signals and long reaction times increased a little during the first half of the night then increased considerably during the second half, generally paralleling decreases in other physiological and behavioral measures of fatigue. Fagerstrom and Lisper (1977) obtained similar results. RT to a tone increased an average of 55 msec over four hours of driving. Furthermore, the increase was greater in silence than with either music or talk, a finding in keeping with other work noting the alerting function of these conditions.

In contrast to the above studies, Mast, Jones and Heimstra (1965) found that the time required to press a brake pedal in response to a red light decreased over four or six hours in a driving simulator. Lisper, Dureman, Ericsson and Karlson (1971) examined RT's to an auditory stimulus during four hours of driving under three conditions: daylight, darkness, and daylight after one night of sleep deprivation. No significant difference between groups was found although there were wide individual differences. In a subsequent study, Lisper et al. (1973) found that over a three-hour period of driving, RT's increased for experienced drivers (annual mileage over $20,000 \mathrm{~km}$ ) whereas it decreased for inexperienced drivers. Using a number of subsidiary tasks including digit sequence detection, digit memory, and RT to a light, other researchers (Brown, 1965, Tickner \& Simmonds, 1966; Brown, Simons \& Tickner, 1977) found no performance decrements even after 12 hours of driving. 
A number of different factors may he partially responsible for the inconclusive findings involving subsidiary tasks. First, it is not always clear which of the two simultaneous tasks is the primary one and which is the subsidiary one. In some cases subjects may completely stop attending to the driving task while they are performing the subsidiary task, and driving measures may not be sensitive enough to reflect any decrement in driving performance for the short time the period of inattention. Second, the type of subsidiary task and the modality of the presentation of signals may affect the results. For example, if the subsidiary task is RT to an auditory signal, it may be easier to perform simultaneously with the primarily visual task of driving than a subsidiary visual RT task. Furthermore, as Brown (1967) suggests, prolonged driving may lead to increased "automization" of control skills which allows attention to be directed elsewhere, leading to better subsidiary task performance toward the latter part of a long drive.

Vigilance. There is a great deal of evidence that performance on long monotonous tasks declines over time (see Mackworth, 1970, or Broadbent, 1971, for good reviews). Vigilance tasks, by definition, introduce a fatigue factor of time-at-task. Some investigations have introduced additional fatigue factors including sleep deprivation, time on a preceding task, or time of day.

Most laboratory vigilance tasks require a subject to detect and respond to signals that have a low probability of occurrence at any point during the session. Over time there is a decrease in the probability of detection of the signals; an increase in speed of signal detection, and often, a decrease in the proportion of false alarms (responses when critical signals were not presented). The following variables have been found to reduce the decrement over time:

$$
\begin{aligned}
& \text { - higher signal rate; } \\
& \text { greater discriminability of critical from non-critical } \\
& \text { signals; } \\
& \text { higher ratio of critical to non-critical signals (greater } \\
& \text { signal probability); } \\
& \text { more complex signals or tasks; } \\
& \text { - } \begin{array}{l}
\text { - } \\
\text { - }
\end{array} \\
& \text { - mowledge of results (provided by feedback during } \\
& \text { scores). }
\end{aligned}
$$

After over 30 hours of sleep deprivation, detection performance was impaired relative to performance after normal sleep on both visual (Wilkinson, 1960) and auditory (Deaton, Tobias \& Wilkinson, 1971)

vigilance tasks. 
Welford (1965) has suggested that some of the decrements in vigilance tasks are the result of boredom rather than "any true fatigue effect". However, as discussed earlier, mental fatigue due to boredom is still a kind of fatigue that may affect performance. Differences between "mental fatigue" and "skill fatigue" on a subsequent visual vigilance task were investigated by Heimstra, Mast and Larrabee (1963). Subjects who had engaged in four hours of mental multiplication missed more signals than those in a control group. However, another group of subjects, who had maintained a minature car on a roadway using a standard steering wheel showed a much smaller, but still significant decrement. Though the distinction between mental and skill fatigue as different kinds of fatigue may not be warranted, the amount of mental effort required by the task may be important in determining subsequent performance decrements.

Circadian rhythms have also been found to affect vigilance performance. Blake (1967) noted that performance on an auditory tone detection task improved during the day ( $8 \mathrm{a} . \mathrm{m}$. to $9 \mathrm{p.m}$.$) except for a$ "post-lunch" dip. However, not all investigators have found circadian patterns in vigilance performance (Thackray, Bailey \& Touchstone, $1975)$.

Selective attention and recognition. Target detection tasks, in general, show decrements with fatigue. Drucker, Cannon and Ware (1969) examined performance on a target detection task over 48 hours. sleep deprived subjects performed worse than subjects who were allowed to sleep. Also, for the sleep-deprived subjects, performance was worse during normal waking hours regardless of time of day, and this difference was magnified as time went on.

Target-detection performance also suffers after four hours of mental multiplication. Heimstra, Mast and Larrabe (1963) found that the greatest difference between fatigue and control conditions was for detection tasks of moderate difficulty.

Various aspects of eye movement behavior have been examined in relation to fatigue. Kaluger and Smith (1970) recorded eye-movement patterns of subjects driving a car around a track before and after one night without sleep. After sleep deprivation, drivers displayed less active eye-movement patterns, often fixating their gaze on the right side of the road rather than monitoring the road ahead. Also, fatigued drivers exhibited more pursuit eye movement and fewer saccades, indicating less active search of the environment. Rackoff and Rockwell (1975) found that drivers exhibited more active scanning patterns during the day than at night. Day-night differences may, however, be due to differences in time awake, circadian cycle, or simply differences in illumination. According to Rockwell (1972), fatigue leads to a narrowing in the field of visual search, as does alcohol. Fruhstorfer et al. (1977) reported that toward the end of a three-hour driving task, drivers had difficulty maintaining fixation. Fatigue also leads to increases in the number and duration of eye blinks (Beideman \& Stern, 1977; Forbes, Katz, Cullen \& Deterline, 
1958; Fruhstorfer et al., 1977; Kaluger \& Smith, 1972). These findings of changes in eye movement patterns indicate that fatigue causes reductions in the rate of information processing and in the ability to maintain attention.

\subsection{Decision Making}

Psychologists have often noticed deterioration in informationprocessing and decision-making over time at a task.

Judgment of hazard. Brown, Tickner and Simmonds (1970) examined the effects of prolonged driving on drivers' criteria for passing other vehicles. They found a significant increase in the percentage of attempted passes that were risky toward the end of the 12-hour drive. Platt (1964) also noted less reluctance to take risks (indicated by an increase in tracking tolerance and sometimes an increase in vehicle speed) over time on a 1200-mile drive. Because Platt's finding is based on one trip by a two-man team, it may have limited generalizability.

Problem-solving. One phenomenon that was repeatedly noticed by Bills (1931, 1935a, 1935b) was transient periods of lapses in attention, which increased in frequency and duration over time at a task. Bills used fairly simple continuous tasks including mental arithmetic, color naming, and letter-number substitutions, and measured the time from stimulus presentation to response. The lapses of attention, or "mental blocks" as he called them, resulted in abnormally long reaction times (RT's) (defined operationally as RT's greater than twice the mean RT). Bills proposed the hypothesis that mental blocks were due to periods of reactive failure of central nervous system processes which could be considered as forced pauses facilitating recovery and preventing a complete breakdown in mental performance. Supporting this hypothesis, Bills found that short rest periods almost completely eliminiated response blocking. A review of response blocking (Teichner, 1968) reported that sleep loss increases the frequency with which blocks occur.

Bugge, Opstad \& Magnus (1979) tested performance on three information processing tasks over a five-day period of sleep deprivation and heavy physical work. All tests showed substantial impairment over the test period and slightly lowered performance at night than during the day. However, it is not possible to separate the effects of environmental changes, psychological stress and work from sleep deprivation. Furthermore, there was no control group to examine circadian changes without sleep deprivation.

\subsection{Motor and Sensorimotor Control}

Neuromuscular control. Some early studies with fatigue due to time driving have examined changes in postural steadiness. Although these studies used a modified Whipple steadiness test rather than the 
Romberg test usually used in alcohol research, the results are conparable. Ryan and Warner (1936) found that postural steadiness decreased an average of $8.3 \%$ after a 300-mile open road drive ana showed no change for a control group who did not drive. Similarly, Jones et al. (1941) found that postural steadiness decreased as a function of hours driven without sleep. Lauer and Suhr (1959) found no significant differences in postural steadiness between a group of subjects who drove continuously and another group who received 15minute rest pauses every hour. The rest pauses may not have been sufficient to counteract the change due to time driving.

Motor control. No work directly comparable to that with alcohol has been done examining ocular-motor control. Some evidence has been found (Kennedy, 1977) that the occurrence of nystagmus eye movements in response to vestibular stimulation may be related to arousal. Kennedy's study showed that there was a significant correlation between nystagmus movements and vigilance performance.

Reaction time. The most commonly reported change due to fatigue in reaction time (RT) tasks is an increase in the variability of response times. Yajima et al. (1976) found no change in mean simple or choice RT over 10 hours of driving, but RT variance increased with time. Similarly, long periods of sleep deprivation (five to eight days) produced unevenness of performance on simple RT tasks, which was greater for longer durations. This unevenness was regarded as "the result of blocks or brief periods of no response which increase in frequency and duration with continued mental work, performance between blocks being maintained at the initial level or close to it" (p. 1 ). This observation is similar to that of Bills, discussed above.

Schmidtke, (1965) reports that mental work for four hours has a greater effect on RT tasks as the number of choice alternatives is increased. From this he concludes that the fatigue produced by mental work affects information processing rather than neuro-muscular processes. Wilkinson (1961) examined the effect of 30 hours of sleep deprivation and knowledge of results on the five choice serial RT task (for a 30-minute period). Sleep loss caused a reduction in correct responses and an increase in gaps about five minutes into the experimental period, with the performance deterioration continuing as the test proceeded. Continuous feedback (knowledge of results) counteracted the performance decrement caused by sleep loss. Williams et al. (1959) found that knowledge of results counteracts sleep loss only when there is an incentive character to it--implicating motivational factors. Wilkinson (1965) notes that much early research on sleep deprivation failed to find any task decrements. The reason for those results, he believes, is that those tests were always short and that (for short periods of time) subjects are able to perform at normal levels. On the serial RT task, no impairment was evident during the first five minutes.

Romansky (1978) examined performance on auditory and visual choice RT tasks over a three-hour period under normal and stressful (moderate heat and noise levels) conditions. Overall performance was 
poorer in the stress than the non-stress conditions on both accuracy and latency measures (except latency in the auditory task). Performance deteriorated over time in both conditions, with greater change in the stress environment and during the second half of the session.

Kleitman and his associates (Kleitman, Titelbaum, \& Feiveson, 1938) Found that both simple and choice RT performance exhibited a circadian pattern, with the change more marked in the choice RT situation. On tests of six subjects, RT decreased progressively during the morning and early evening; performance in most cases parallelled the circadian temperature cycle. Blake (1967), in contrast, found no change in simple RT as a function of time of day.

Tracking. Drucker, Cannon, and Ware (1969) examined tracking a continuously winding road on a driving simulator over 48 hours with and without sleep deprivation. The sleep-deprived group performed significantly worse overall than the control group. The control group maintained a consistently high level of performance throughout the 48 hours while the sleep-deprived subjects' performance deteriorated during the nights (more the second night) and recovered during the day.

Mast, Jones and Heimstra, (1965) looked at the effects of timeon-task on tracking performance in a driving simulator over both four- and six-hour periods. Tracking performance was significantly worse in the last than the first hour of the six-hour period, but not for the four-hour period. In a control condition subjects operated the simulator only during the first and last hours of the session. In this condition, there was no difference in performance between the Eirst and last hours.

Tracking performance was measured under static (no motion) and dynamic (angular acceleration) conditions after more than 24 hours of sleep deprivation. Impairment decrements were found which were greater after longer amounts of sleep deprivation ( 55 vs. 34 hours) and with motion (Collins, 1977).

Heimstra (1970) and Heimstra, Jones, and DeKock (1965) examined performance with and without "stress fatigue" (low voltage electric shock). Performance was poorer in the shock conditions.

\subsection{Overall Vehicle control}

The effects of fatigue on overall vehicle control have been examined in simulator studies, on closed-course driving tasks, and in open-road driving studies. Alcohol studies, in contrast, rarely use open-road tests. Some measures are recorded continuously over a driving period, whereas other measures are pre-test and post-test comparisons. Primarily, sleep deprivation and driving time have been the fatigue factors investigated, but time of day and fatigue due to stress have also been used. 
Two studies have used suhjective evaluation of performance by observers as dependent measures. Brown (1967) tested six Arivers in the morning and afternoon on a route in city traffic. on one occasion, the subjects drove continuously for seven hours between the test, and on another occasion they performed their routine jobs. Observer ratings of competence using controls, anticipating traffic changes and courtesy factors indicated an increase in errors from morning to afternoon for those drivers who had continued driving in between. Mackie and O'Hanlon (1977) examined changes in numbers of technical errors over time driving and found large individual variability but no significant changes.

Many early studies used pre- and post-tests to assess performance decrements due to time driving. Of these, two tested performance on actual driving maneuvers rather than on other psychomotor tests. Herbert and Jaynes (1964) tested 180 subjects on a set of driving maneuvers including parking, backing, and following a precise path, both before and after $0,1,3,7$, or 9 hours driving. Performance declined in the post-test as a direct function of time spent driving. Lauer and Suhr (1959), however, found no difference in parking efficiency before and after a three-hour open-road drive, regardless of whether or not subjects received a rest pause half-way through.

Longitudinal control measures including brake usage and velocity control have been examined as a function of fatigue, primarily time driving and sleep deprivation. Forbes et al. (1958) found that extreme sleep deprivation (24-36 hours) resulted in increases in unnecessary speed changes over time on the open road and less accurate control of speed than under rested conditions. Moderate sleep deprivation (only four hours sleep for three nights) did not produce any difference compared to normal conditions. Platt and Fedderson (1964), in an examination of the effect of work-related stress on driving, found that evening runs showed higher and more variable speed change rates and more accelerator reversals than morning runs. A number of other variables, including time of day, light vs. dark conditions, and traffic density also differed between the two test runs for each subject and need to be considered in drawing conclusions from this study.

Most other studies, however, have not found significant changes in velocity control associated with time-on-task. Brown (1965a; Brown et al., 1966, 1977) found no differences in velocity control over 8 or 12 hour drives. Their measurements were only taken periodically, however, and consequently may not be as sensitive as more sophisticated measures used more recently. Greenshields (1966) found no changes in velocity control over time in a four-hour, open-road study, but did notice that there was a great deal of variability in velocity changes within subjects. In a study of changes over a night of driving, Riemersma et al. (1977) found that variability in speed maintenance increased, but not until the second half of the night, and then this was only an overall trend. Two further open-road studies found no changes in velocity control over time or after rest pauses (Lauer \& Suhr, 1959; Safford \& Rockwell, 1967). 
The results from simulator studies with regard to this variable are inconsistent. Sussman and Morris (1970) report no change over four hours, while Mast et al. (1966) found a deterioration in velocity control over six hours.

Increased brake usage might denote problems with velocity control. None of the investigators who examined brake usage measures found any effects (Brown, 1965; Brown et al., 1966, 1967; Greenshields, 1966; Lauer \& Suhr, 1959).

Lateral vehicle control including road position and steering behavior are frequently examined types of performance for evaluating fatigue effects. The dependent measures of steering behavior have included subjective observation of steering errors and continuous recording of steering wheel movements using sensing devices. As would be expected, the latter method provides the most accurate measures, and it is the only way to differentiate accurately between coarse and fine steering adjustments, or to calculate the average size of steering movements during a time interval.

Alert, unstressed drivers make many fine steering adjustments in smoothly tracking the highway geometry. In general, steering behavior becomes less accurate and more variable with increases in fatigue. Some studies have counted overall numbers of steering wheel reversals, while others have counted only fine movements (usually $2^{\circ}$ ) or only coarse movements (usually $12^{\circ}$ ), and still others have counted fine and coarse movements separately. Many studies have found that the steering wheel reversal rate decreases over time on the road (Forbes et al., 1958; Greenshields, 1966; Lauer \& Suhr, 1959; Platt \& Fedderson, 1964; Sugarman \& Cozad, 1972), on a simulator (McFarland \& Mosley, 1954; Sussman \& Morris, 1970), and on a closed circuit track (Michant \& Pottier, 1964). Brown and his associates (Brown, 1965, Brown et al., 1966, 1967) and Safford and Rockwell (1967) did not find significant changes. Sugarman and Cozad (1972) further noted that an overall measure of steering position tended to increase over time as the frequency of small steering movements $\left(2^{\circ}\right)$ decreased.

Steering errors, defined as number of drifts to the side of the lane, have been found to increase over time in a simulator (Dureman \& Boden, 1972; Ellingstrad \& Heimstra, 1970; Sussman \& Morris, 1970) and on the open-road (Forbes et al., 1958; Lauer \& Suhr, 1959; Mackie \& O'Hanlon, 1977; O'Hanlan \& Kelly, 1977).

Lane position variability, measured by a light-sensing device attached to the vehicle which determines position within the lane relative to the center line or shoulder, has been examined in several recent studies. Prolonged driving has led to increased variability in lane position over time in all cases in which it was measured (Caille \& Bassano, 1977; Riemersma et al., 1977a, 1977b; Sugarman \& Cozad, 1972). 


\subsection{A COMPARISON OF ALCOHOL AND FATIGUE EFFECTS ON DRIVER PERFORMANCE}

Since very little experimental work has been done examining the interactions of alcohol and fatigue, the effects of alcohol alone and fatique alone can he compared to find similarities. Performances which are affected in similar ways hy the two factors indicate possible areas in which synergistic interactions may be found. By the same reasoning, performances which are affected in different ways indicate possible antagonistic interactions. of course, further experimentation is necessary to determine if these interactions actually occur.

This chaper will compare the effects of alcohol and fatigue (described in Chapters 4 and 5, respectively) on driver performance, following the same organization as those two chapers followed.

\subsection{Sensory Processes}

While the effect of alcohol on sensory processes has been explored extensively, relatively little research has attempted to examine the effect of fatigue on the senses. Therefore, a comparison of the role of alcohol and fatigue is invariably constrained by the present limited state of knowledge. Nonetheless, the available findings tend to suggest that alcohol and fatigue display similar effects on the sensory processes.

Static and dynamic visual acuity. While static visual acuity has been found fairly resistant to alcohol except at very high doses, there is some evidence that it may be impaired by fatigue elicited by continuous time at a task. However, other fatigue studies have failed to demonstrate any degraded static visual acuity.

On the other hand, dynamic visual acuity, which is probably more relevant to actual driving performance, has been found more consistently susceptible to the influences of both alcohol and fatigue. In particular, dynamic visual acuity was found vulnerable to both low levels of alcohol and to fatigue brought on by aircraft piloting, physical exertion, and sleep deprivation.

Visual field. Although empirical evidence has been more substantial for alcohol than for fatigue, it suggests that both factors may induce a narrowing of the visual field or "tunnel vision". However, this peripheral impairment is more likely to occur as a function of attentional limitations rather than as the consequence of any real sensory impairment. In this vein, narrowing of the visual field will result under the influence of both alcohol and fatigue only to the extent that attention is more directly focused on central vision, neglecting more peripheral vision. 
Adaptation and brightness sensitivity. While it was found that dark adaptation is impaired at high levels of alcohol, fatigue's efffect on light and dark adaptation still awaits investigation. However, because fatigue is more frequently present during dark nighttime hours, it is possible that it may interact with alcohol's impairing effect on dark adaptation. This possiblility has not yet been tested in an experimental setting.

Glare resistance. A study of alcohol's effect on glare resistance has produced inconsistent findings at best, while fatigue's role has not yet been considered in any known research study.

Temporal Acuity. Temporal acuity, unlike other aspects of the sensory processes, has been subjected to considerable investigations, especially involving the role of fatigue. The critical flicker fusion threshold (CFF), commonly used to measure temporal acuity, has been reported to be lowered both by fatigue and by high levels of alcohol. The lowering of CFF has been found consistently in many different alcohol studies and in fatigue studies with long and/or monotonous task conditions. It also shows a circadian rhythm. However, at BAC levels below $0.08 \%$, alcohol's effect on the CFF is not as clear.

\section{2 Perception and Attention}

Intensive attention and vigilance. Physiological changes have been measured after both alcohol ingestion and fatiguing conditions. Since many physiological measures can be recorded continuously, changes over time can be examined. Also, it was hoped that these measures might provide indications of fatigue that were uncontaminated by motivation or knowledge of the results of performance. However, these measures have not proven as valuable as was anticipated. Results are not always consistent between studies, and many momentary changes in the environment create greater physiological changes than the changes due to time-on-task. In spite of these problems, some general trends have been found. Both alcohol (at moderate and high levels) and fatigue have depressant effects on the central nervous system. Moderate and large doses of alcohol, and fatigue from sleep deprivation or long-term driving reduce electroencephalogram (EEG) frequency; that is, slower alpha waves are found more frequently than under normal alert conditions when higher-frequency beta and gamma waves predominate. Individual variation is great, however, and with alcohol, there is a poor correlation between EEG pattern and behavioral signs of intoxication.

Electrical activity measured directly from the sensory cortex areas usually shows large potential changes (called evoked potentials) after direct sensory stimulation. When this occurs, alpha activity is suppressed. Alcohol has been found to diminish the suppression of alpha activity, reduce evoked potentials, and increase their latency. A study by Peeke et al. (1980), which is presented in Chapter 7, discusses some alcohol and fatigue interactions on EEG and evoked potential measures. Few other studies have looked at the effects of fatigue on evoked potential. 
Heart rate generally decreases in mean rate and increases in variability over long drives, but traffic events such as ruming orer lane markers momentarily reverse those trends. In contrast, heart rate increases after alcohol ingestion (Wallgren \& Barry, 1970; Peeke et al., 1980). This is discussed in Chapter 7.

Correlations between pupil size and information-processing demands have been found in studies of alertness. Thus, this might be a good measure to examine after alcohol ingestion.

Vigilance. A comparison of several studies involving the roles of alcohol and fatigue in vigilance performance reveals clear-cut differential effects. Alcohol has repeatedly been noted for its relatively small influence on vigilance. Fatigue, on the other hand, has consistently led to degraded vigilance performance. Moskowitz (1979) has even attributed vigilance decrements in the few alcohol studies that reported this finding to the confounding effect of fatigue. This detrimenal fatigue effect has been reported when fatigue is defined according to hours of sleep deprivation, time on task, and time of day/circadian rhythms. However, it must be noted that this vigilance decrement is not immutable, and may be reversed, or at least reduced, with the introduction of several factors including motivating and/or more complex tasks.

Selective attention and recognition. Selective attention and recognition appear highly sensitive to the impairing effects of both alcohol and fatigue. However, the nature of these impairments is uncertain. The results of several studies suggest that alcohol and fatigue may act to increase the amount of time required to process the information presented in a task, thus impairing performance on that task. However, it has also been suggested that alcohol may impair the amount of information that can be processed rather than the rate of the processing, but it is most likely that alcohol influences both these functions.

This information processing impairment has been demonstrated almost universally in alcohol studies where the task requirements demand divided attention, such as in time-sharing tasks. In these studies, alcohol appears to reduce the attentional capacity of the subject. Fatigue has also clearly exhibited an impairing effect in several studies requiring performance on target detection tasks. However, fatigue studies requiring subsidiary detection tasks have reported inconsistent findings. In one study, no performance decrements were found even after 12-hours of driving (Lisper, 1973). While several explanations have attempted to account for this inconsistency, it appears that fatigue's effect on selective attention may be qualitatively different from alcohol's effect.

Eye movement studies have provided additional evidence to indicate selective attention impairment under the influence of both alcohol and fatigue. Both these factors have been held responsible for eliciting a change in movement patterns reflecting less active, 
narrowed, and less efficient visual search behavior. Characterizing this change in eye movement patterns is an increase in fixation duration under the influences of both alcohol and fatigue. Intoxicated subjects have additionally displayed a decrease in the frequency of fixation while fatigued subjects have exhibited more pursuit eye movements with fewer saccades and an increase in both the number and duration of eye blinks. Moreover, unusually long fixations, gazes or blocks suggesting a momentary cessation or attenuation of attention have been noted in fatigued and intoxicated subjects. These changes in eye movement patterns support the notion that both alcohol and fatigue cause a reduction in the rate of information processing, a function extremely relevant to driving performance. However, there is the possibility that fatigue may reduce the ability to maintain one's attention while alcohol may serve rather to reduce the attentional capacity of the driver. However, this has not yet been confirmed through experimentation.

\subsection{Decision-Making}

While alcohol has been implicated in the impairment of juagments regarding time and distance and memory, fatigue's role in these types of decision-making processes has not yet been evaluated. Thus, comparisons of decision-making performance have been limited to judgments of hazard and problem-solving.

Judgement of Hazard. While fatigued drivers appear more likely to attempt risky maneuvers while driving, this finding cannot necessarily be generalized to include intoxicated drivers. Intoxicated arivers did display a tendency to choose risky alternatives in hypothetical situations, but they were much more cautious while driving in real traffic. Evidence does indicate, however, that intoxicated drivers are more likely to judge their driving performance as normal or adequate when in fact it is clearly impaired.

Problem-solving. While both alcohol and fatigue have been found to cause problem-solving impairment, the nature of this impairment is somewhat different. Fatigue, especially that operationally defined as continuous time at a task or as hours of sleep deprivation, has been found to result in variability in performance on problemsolving tasks. Periods of normal performance are interspersed with mental blocks, or lapses in attention, which result in abnormally long reaction times.

Alcohol, on the other hand, has been found more likely to impair accuracy than speed of performance and to impair performance on complex, more difficult tasks while having little or no effect on. simpler tasks. However, intellectual functions are fairly insensitive to low levels of alcohol and may, in fact, sometimes be facilitated by low doses. 


\subsection{Motor and Sensorimotor Control}

Neuromuscular control. Standing steadiness tests have been the most common measure of neuromuscular control investigated. Both alcohol and fatigue have led to impairments in standing steadiness. Since the Romberg Test of standing steadiness is frequently used as a test for intoxication, it is important to note that fatigue might also affect this measure.

Motor control. Alcohol has been found to produce very characteristic patterns of nystagmus eye movements under certain conditions. This pattern is so distinctive that it has led to attempts to develop a psychophysical test of intoxication (Tharp, Moskowitz \& Burns, 1980) based on it. The work by Kennedy (1977) showing that the occurrence of nystagmus eye movements may be related to arousal indicates that fatigue might cause changes in ocular-motor control similar to those caused by alcohol ingestion.

Reaction time. The time to respond to a stimulus includes the time for sensory registration of the stimulus, whatever decision processes are necessary, and the execution of an actual motor response. The portion of reaction time (RT) that goes to each of those three processes cannot always be determined.

If sensory processes are intact, increases in simple RT most likely indicate motor impairment. Some studies found small increases (less than 10\%) in simple RT with alcohol, but there is no general agreement. When effects were found, they resulted from high BAC levels. Fatigue, in general, does not affect simple RT, unless sustained performance is required. Time of day affected RT in one study, but not in another.

When RT tasks are more complex, requiring a greater involvement of attention or decision-making, larger decrements are found for both alcohol and fatigue. The demands are increased either by increasing the number of choice alternatives or by testing RT concurrently with another task. Both alcohol and fatigue increase choice RT more than simple RT. Fatigue primarily increases the variability of response times, especially when the RT task must be performed over long periods of time.

Performance on RT tasks suffers to a greater extent when there are concurrent demands under conditions of either intoxication or fatigue, implying that information processing capacity may be diminished. Some research examining alcohol effects have taken speedaccuracy tradeoffs into account, and found that response speed can only be maintained at the expense of accuracy. These tradeoffs still need to be examined in fatigue work. The RT decrements found with fatigue can be overcome to a great extent by motivation (e.g., knowledge of results). Motivation can overcome some alcohol decrements, but not enough work has been done to determine if choice RT decrements can be overcome. 
Tracking. An important characteristic of tracking tasks is that they must be performed continuously. Of tracking tasks, compensatory tracking is less demanding than pursuit tracking, because the former requires monitoring only one source while the latter requires monitoring two or more sources. In general, compensatory tracking is impaired only when it is performed concurrently with another task. Pursuit tracking, in contrast, is almost always impaired even with BAC levels as low as $0.05 \%$. Greater impairment occurs with higher BAC levels and with divided attention. Since simple tracking tasks are not impaired with alcohol, the impairment is most likely not of a neuromuscular nature. Furthermore, since increases in informationprocessing demands increase the impairment, attention and information-processing capacity are implicated.

Fatigue consistently impairs tracking performance. Sleep deprivation, time on the task, and stress (from low voltage electric shocks) all have been found to negatively affect tracking. The continuous nature of tracking is most likely the reason for its impairment with fatigue. Fatigue can be overridden in most tasks for short periods of time; however, when the task must be continued for longer periods performance usually suffers.

\section{5 Overall Vehicle Control}

Rough performance measures such as amount of time to complete a task, number of pylons hit on a course, or subjective judgments of ability have in some cases shown decrements with both alcohol and fatigue. Different studies are not always in agreement, however, and other more sensitive measures are more likely to yield more replicable results. Time to complete a task or course may be a useful measure, but only when tradeoffs between speed and accuracy are taken into account. Pre- and post-tests of driving maneuvers have been used primarily in fatigue studies and have shown decrements due to time driving. These tests also must be interpreted cautiously because other studies have shown that fatigue decrements can often be overridden in short tests when attention can be concentrated on one task.

Longitudinal control measures including braking and velocity maintenance have proven vulnerable to both alcohol and fatigue. Alcohol has been found to impair smooth braking and increase braking time; however, Attwood et al. (1980) did not find performance on a closed-course stopping task associated with intoxication. No studies that have examined braking as a function of fatigue have found any effects.

Velocity-control appears sensitive to both alcohol and fatigue, although in both cases conflicting results have been obtained. Accelerator reversals are more frequent and other measures of speed change rate are more variable with moderate to high levels of alcohol and with fatigue from sleep deprivation and work-related stress. Time driving has in some cases led to more variability in velocity control, 
but not in others. A closer-course car-following task (Attwood et al., 1980) has proven very sensitive to alcohol ingestion, showing impairments even at very low doses. Since this task requires both velocity control and stopping ability, it appears to be a valuable task for further research with fatigue or alcohol and fatigue in combination.

Lateral vehicle control, including steering behavior and lane position variability measures, display effects under both alcohol and fatique. In general, alcohol leads to increases in the number of steering wheel reversals made while fatigue causes decreases. Alert, unstressed drivers make many fine steering wheel reversals to maintain the vehicle on course, with large reversals necessary only for major corrections of course. Some studies have differentiated between fine and coarse steering wheel reversals, and although this is an area that needs further investigation, some general conclusions can be tentatively advanced. Fatigue appears to increase coarse steering reversals while decreasing fine reversals. Alcohol, in contrast, just increases all steering activity, rather than causing a change in the average size of steering corrections.

Lane position variability, as measured by sensing devices, has proven to be one of the most sensitive measures of impairment due to alcohol or fatigue (Attwood et al., 1980) but it requires expensive equipment and conseguently is not widely used. Lane position can also be measured as the number of drifts to the side of the lane, but that measure is not as sensitive. In most studies, both alcohol and fatigue have caused increases in lane position variability.

\subsection{Summary of Similarities and Differences}

The main similarities in the effects of alcohol and fatigue are:

- neither has a large effect on the sensory. aspects of vision;

- both decrease information-processing capacity and rate:

both increase the difficulty of controlling the speed and position of the vehicle; and

- there are large individual differences in the effects of both.

The main differences are:

- vigilance is more impaired by fatigue than alcohol;

fatigue impairs simple tasks more than complex ones, while alcohol impairs complex ones more than simple ones; and

alcohol increases steering wheel reversals whereas fatigue decreases them. 


\subsection{ALCOHOL AND FATIGUE INTIRACTTONS}

Despite the large amount of research on alcohol alone and fatigue alone, very few studies have examined the combined effect of these two factors on driving or driving-related performance. In our search of the literature, we found only six relevant studies. Since this research provides findings that directly addresses the issue of whether fatigue modifies the effect of alcohol, these six studies will be described in detail. In this way, the methodology of each study can be evaluated and the similarities and differences between studies noted. Thus, this chapter is not organized by performance types as the previous three chapters were.

Four of the studies involving both alcohol and fatigue used laboratory sensorimotor or information-processing tasks; the fifth used a closed course driving task; and the sixth used a simulator task. Four of the studies examined sleep deprivation as the fatigue factor; the fifth examined day versus night performance; and the sixth examined time on the task.

Gibbs (1968) looked at the effect of alcohol consumption and sleep loss, alone and in combination, on decision processes and movement precision using an instrument (called a stressalyzer) on which a light appeared at one of five positions on a panel. The person being tested had to align a pointer with the target light using a handwheel. The device measured the time to begin movement, the number of decision errors (movements in the wrong direction), and other measures related to the speed and precision of movement. This was not a wellcontrolled study: many of the details of the experiments were not reported, only men were tested, comparisons were among different groups, and no statistical tests were used on which to base conclusions. However, since the study looks at sleep deprivation and alcohol consumption together, the results are worth reporting.

In all the experiments described by Gibbs, there were great differences among individuals, both in innate ability and in tolerance to the different types of stress. With increasing intoxication, most men increased the time necessary to make a decision while maintaining the same level of accuracy. A small number reduced their decision time and showed a large increase in decision errors. Some subjects were impaired with a breathalyzer reading of $0.03 \%$, while a larger proportion were impaired with a reading of $0.05 \%$

A group of sober men were deprived of sleep and tested every four hours over a 48-hour period. The overall efficiency of the group had deteriorated $50 \%$ after 20 hours without sleep. However, about 25\% of the subjects showed substantial deterioration in performance after 16 hours.

Another group of men were deprived of sleep for 36 hours and consumed enough alcohol to produce an average breathalyzer reading of 
0.08 o hy the end of the experimental period.

Five subjects improved over the test period and three deteriorated.

Wilkinson and Colquhoun (1968) examined the effect of alcohol and sleep deprivation on a five-choice serial reaction time task. Each participant was tested for 30 minutes in each of the four combinations of alcohol (70 CC of 90.5 proof alcohol producing BAC's of 0.01 to $0.07 \%$ ) vs. placebo and normal sleep vs. 30 hours of sleep deprivation. Reaction time, number of errors, and number of gaps over 1.5 seconds between responses were recorded. Both sleep deprivation alone and alcohol alone impaired performance. The effect of alcohol on accuracy was greater following normal sleep than following sleep deprivation. However when subjects were divided into two groups based on whether BAC was greater than or less than 0.032\%, further differences appeared. For the low BAC group, loss of sleep reduced the adverse effect of alcohol on performance speed, whereas for the high BAC group the adverse effect was increased by sleep loss. Thus, the interaction was antagonistic at low doses and synergistic at moderate doses.

In contrast to the Gibbs (1968) study, that of Wilkinson and Colquhoun (1968) was a well-designed and carefully reported study. However, the alcohol dosage used was not dependent on body weight, so that a range of BAC's were produced. The post-hoc division of subjects into high and low BAC's did alleviate the potential problem, and pointed out the important difference between high and low alcohol levels in their interaction with sleep loss.

Rutenfranz and Singer (1967, cited in Wallgren and Barry, 1970, p. 354) tested the sensorimotor performance of six subjects over a 12-hour period during the day (beginning at 8:30 a.m.) or during the night (beginning at 8:30 p.m.). Recovery from the detrimental effect of $1 \mathrm{~g} / \mathrm{kg}$ of alcohol on performance, which gave rise to a peak BAC of $0.08-0.15 \%$, was greatly retarded at night. These results indicate that the detrimental effects of alcohol are enhanced at night during prolonged testing, though it was noted that people under the influence of alcohol and fatigue were able to "pull themselves together" temporarily. This study must be interpreted cautiously, since there was no non-drug control condition, and the day-night differences could be just as large without alcohol as with.

Huntley and Centybear (1974) examined effects of alcohol (BAC's ranged from $0.074 \%$ to $0.094 \%$ during testing vs. placebo) and sleep deprivation ( 29 hours mean awake time vs. six hours for normal sleep condition) on closed-course driving in an instrumented car. Subjects drove through a serpentine pathway defined by pylons, attempting to maintain a constant speed of either 10 or $15 \mathrm{~m} . \mathrm{p} . \mathrm{h}$., to drive smoothly, and not to hit any pylons. The following measures were recorded: fine steering reversals (two degrees), coarse steering reversals (12 degrees), brake applications, accelerator reversals, total time the car moved forward at more than five m.p.h., and the number of times the speed of the car changed more than two m.p.h. 
Alcohol caused significant increases in control-use rate for coarse steering, fine steering and accelerator use. It also caused an increase in speed-change rate. Control-use and speed-change rates were all lower following sleep deprivation than following a normal night's sleep, but the differences were not great and those for control-use rates were not significant.

A sleep-by-beverage interaction was found for coarse-steering-alcohol increased coarse steering rate following normal sleep, but not following sleep deprivation. This antagonistic interaction was the only interaction reported; no synergistic interactions were found.

Peeke, Calloway, Jones, Stone and Doyle (1980) examined the effect of combining sleep deprivation and moderate alcohol consumption on autonomic, behavioral, central nervous system (CNS) and subjective measures in a categorization and a complex choice reaction time task. Three levels of alcohol consumption (moderate $.90 \mathrm{ml} / \mathrm{kg}$ of $95 \%$ ethanol, low $0.45 \mathrm{ml} / \mathrm{kg}$, and placebo $0 \mathrm{ml} / \mathrm{kg}$ ) and two levels of sleep deprivation ( $O$ hours and 26 hours) were used. The moderate alcohol level yielded a mean BAC of $0.063 \%$ when testing began and the low dose yielded $0.034 \%$ Response measures included reaction time (RT), error rate, and gap frequency as performance measures; EEG, evoked potentials, and heart rate; and self-ratings of alertness, anxiety and amount of intoxication.

Alcohol alone or sleep deprivation alone produced mild performance impairments, decreased alertness and reduced amplitude and increased latency of cortical evoked potential components. Alcohol increased heart rate and sleep deprivation increased anxiety.

Interactions were synergistic in some cases and antagonistic in others, though antagonistic effects were more common. There were more interactions for moderate amounts of alcohol and sleep deprivation than for small amounts, and in this study the type of interaction never differed between low and moderate doses. Furthermore, interactions were found for some measures that showed no effects for either treatment alone. In the categorization task, errors increased synergistically when sleep deprivation and alcohol were combined, whereas RT decreased antagonistically compared to the slowing of RT's with either treatment alone. Late components of the evoked potential showed long latencies for sleep deprivation and moderate alcohol doses, a synergistic effect compared to alcohol or sleep deprivation alone. All other interactions were antagonistic. Early evoked potential components showed an increase in latency as a result of sleep deprivation alone, but a decrease when alcohol was combined with sleep deprivation. The amplitude of the $\mathrm{P}_{2} 00$ - N300 evoked potential component--which has been related to drowsiness and the onset of sleep (Weitzman and Kremen, 1965) showed high amplitude for sleep deprivation alone but declined when alcohol was combined with sleep deprivation. The increase in heart rate from alcohol alone was diminished when sleep deprivation and alcohol were both present. The subjective feeling of anxiety resulting from sleep deprivation was mitigated when alcohol was also present. 
A study by Nelson, Ladan, and Carlson (1979) looked at the role of alcohol upon the development of fatigue from continuous time at a task. The subjects' task was to operate the simulator until they "perceived the task to be so aversive they "wished to stop" " This was a well-controlled study using a within-subjects design to test three levels of BAC: no alcohol, low alcohol (BAC less than 0.08\%) and high alcohol (BAC greater than 0.08\%). Condition order was random, and a double-blind procedure was used. Drivers watched a videotape projection of a roadway and attempted to follow the road with the wheel, maintain a prescribed speed, and respond appropriately to hazards and markers. In addition to measuring total time spent driving, heart rate was measured, periodic ratings of fatigue (Pearson-Byars Feeling Tone Check List) and mood (Nowl is Mood Adjective Checklist) were obtained, responses to critical events were recorded by an observer, and driver comments were recorded and classified.

The main finding was that driving duration was shorter for higher BAC levels. There were no differences in responses to critical events for alcohol level or point during driving. However, the measure here was observer categorization of the responses as appropriate rather than objective measures of reaction time or steering accuracy. Driving duration was shorter for higher BAC levels. Also, since response measures were not recorded continuously, subjects could stop doing anything else and respond to the stimulus. Heart rate was higher in the two alcohol conditions than without, but did not change over the course of the driving session. Pearson-Byars scores indicated that fatigue increased as a function of duration; alcohol dose had no effect; nor was there any interaction of dosage with duration. On the General Activation scale of the Mood Adjective Check List, subjects rated themselves as more fatigued with no and high dosages than with low dosages. In all conditions, fatigue ratings were higher after driving than before with the increase smallest in the no-alcohol condition.

One problem in interpreting these results is that the amount of time on the task varied, since subjects stopped at different times. Thus, before and after differences may be variable. Furthermore, objective measures might have shown some effects that were not observed with the imprecise response measures used.

Summary of interaction effects. These six studies give some indications of the possible ways in which fatigue and alcohol can interact. However, the studies are sufficiently different in method, type of performance examined, and fatigue factor examined, that it is difficult to draw many conclusions about the interactions of the two factors on driving performance. Both synergistic and antagonistic interactions were found on different types of performance.

one important finding is that there are large differences in the way alcohol and fatigue affect different individuals. Furthermore, they interact in different ways for different individuals. Gibb's (1968) study particularly points out the importance of individual differences. 
A second general point to be made from these interaction studies is that it is necessary to consider the alcohol dosage. As Wilkinson and Colquhoun (1968) have found, low BAC levels reduced the adverse effect of sleep loss on performance while high BAC's increased it. Peeke et al. (1980) did not find different directions of interactions at high and low alcohol dosages but did find cases in which an interaction occurred at a high dosage when there was no effect at lower dosages.

Another interaction of alcohol and fatigue results because alcohol induces fatigue. The depressant effect of alcohol can lead to inattention, unresponsiveness or passing out. Large amounts can lead to sleep or stupor. Subjective feelings, based on introspective observations and standard questions about mood, emotion or fatigue, after alcohol are primarily pleasant and euphoric moods, and loud, cheerful, friendly behavior (Wallgren and Barry, 1970). However, other reports are of feeling more sleepy, calm and contented. The disinhibitory effects are more common at low alcohol levels and the depressant effects follow if the alcohol level in the body rises to higher levels.

The Nelson et al. (1979) study showed that fatigue ratings increased more after time driving if the subject had consumed alcohol than if sober. Other studies have also found that fatigue rating are greater after alcohol consumption. Goldberg (1966) found that BAC's of 0.0658 increased ratings of feeling tired, sleepy and hazy in comparison with nondrug conditions. Warren and Raynes (1972) compared feelings at $0.05 \%$ and $0.10 \% \mathrm{BAC}$ and found that vigor was more greatly decreased and fatigue more greatly increased at the higher level. Hurst, Radlow, Chubb and Bagley (1969) reported that fatigue decreased slightly one hour after drinking, then increased after another half hour. This evidence suggests that fatigue may result directly from alcohol ingestion, as well as from any of the other fatigue factors discussed in this report, and may partially account for the linkage between the two in many accident reports. 
8.0 ESTIMATES OF THE PROPORTION OF ACCIDENTS CAUSED BY FATIGUE AND ALCOHOL

\subsection{Present Estimated Effects of Alcohol}

The risks of driving while intoxicated have become familiar, well-documented and frightening statistics. Because many reports have already provided detailed and generally consistent accounts of these risks, it would be superflous here to provide anything more than a brief summary of these findings.

Most studies attribute 40 to $55 \%$ of fatal accidents on the road to alcohol intoxication (Filkins, Clark, Rosenblatt, Carlson, Kerlan \& Manson, 1970; Neilson, 1969; Perrine, Waller \& Harris, 1971; Waller, Neilson \& Turkel, 1970). This figure becomes astounding when compared with only 2-3\% of the legally intoxicated drivers who are on the same road at this time, hut are not involved in accidents (Waller et al., 1970; Alcohol Safety Action Program of the National Highway Traffic Safety Administration, 1979). Similarly, it was found that while 5-50\% of drivers involved in any type of crash were legally intoxicated, only 1-3\% of non-crashed drivers were legally intoxicated (Borkenstein, Crowther, Shumate, Zeil \& Zylman, 1964; Perrine et al., 1971; Farris, Malone \& Lilliefors, 1976; McCarroll \& Haddon, 1962; Holcomb, 1938).

Single-vehicle fatal accidents were more likely (55-65\%) to be associated with illegal blood alcohol concentration (BAC) levels than were multiple-vehicle accidents (25-50\%) (Handon \& Brandess, 1959).

Hurst (1973) examined a number of previous epidemiological studies and calculated the relative probabilities of accident involvement as a function of $\mathrm{BAC}$. His work showed that the risk of being involved in an accident also progressively increases as the BAC level

increases. When a BAC level of 0.088 is obtained, the probability of involvement in an accident begins to rise sharply. At a BAC of $0.10 \%$, this risk becomes 6-12 times as high as at a BAC of zero. Of all the fatally injured drivers reported, 29-43\% had BAC's of $0.15 \%$ or greater (Waller et al., 1970; Perrine, Walker \& Harris, 1971; Neilson, 1969). on the other hand, of non-fatal accidents reported, only 6-15\% of the drivers showed a BAC of $0.10 \%$ or more (Lucas et al., 1955;

Borkenstein, et al., 1964). At very low levels of intoxication, the relationship between accident risk and alcohol is less unequivocal. A Grand Rapids, Michigan study, for instance, reported a slight

decrease in accident risks at very low BAC levels (Borkenstein et al., 1964). However, this finding has not been confirmed by other studies.

The risks of driving while intoxicated also vary according to characteristics of the driver such as sex and age. For example, while males are generally more likely to be involved in accidents than females, this difference increases under the influence of alcohol (i.e., 81-88\% of non-drinking drivers were male while $90 \%$ of intoxicated' drivers were male) (Waller et al., 1970; Filkins et al., 
1970). However, Carlson (1972) found that women were involved in accidents at lower BAC levels than males. Both the very youngest, (i.e., below 20) and oldest arivers were also less frequently found intoxicated while driving. However, when younger drivers do drink, they increase their chances of having an accident to a degree higher than that for drinking drivers as a whole (Jones \& Joscelyn, 1978). Carlson (1972) also reported that younger, less-experienced drivers tend to get involved in accidents at lower BAC levels than that for the general driving population.

\subsection{Present Estimated Effects of Fatigue}

Unlike that of alcohol, an assessment of the percentage of highway accidents in which fatigue plays a causal role is, at best, a difficult and clumsy affair. Because fatigue has been neither objectively nor consistently defined, findings will vary according to each arbitrarily chosen definition. Similarly, because no physiological, psychophysical or other objective or subjective measure of fatigue has been established (such as the BAC level for alcohol), there is obviously no way to determine to what extent different levels of fatigue will increase the likelihood of an accident. To make matters worse, the effect of fatigue may often be masked by and attributed to more direct and easily discernible factors. For example, in fatal accidents where the driver has a high BAC level, the possible role of fatigue or sleep may easily be overlooked. Many studies do not even attempt to assess the role of fatigue except when it results in "falling asleep at the wheel". This neglect results in an underestimation of the magnitude of the problem. It especially neglects the substantial contributory role of fatigue in accidents where other causal factors are more directly accountable, such as inattention or excessive speed. Potential accidents due to fatigue, where the drivers "do not run off the roadway far enough to incapacitate their vehicle" may also seldom be reported (Hulbert, 1972). Hulbert cautions that those accidents which have been attributed to fatigue may represent "only the visible tip of a gigantic iceberg constituting a national driving problem about which very little is known" (Hulbert, 1972, p. 228 ).

With this caution in mind, Hulbert (1972) states that fatigue or drowsiness has been found to directly cause at least 35-50\% of the highway fatalities. Similarly, the Pennsylvania Turnpike Commission has found that drowsiness or falling sleep was "one of the two or three most frequently occurring driver behaviors in highway traffic accidents..." (Forbes et al., 1957, p. 21). They estimated that sleep played a role in from 13 to 20 more percent of Turnpike accidents in each year studied, while it was also implicated in 35 or more percent of the accidents in which a fatality accurred (Forbes, 1957, p. 21). Harris et al., (1972) also hinted at the magnitude of the problem in their statistics on commercial vehicle accidents. Citing a survey performed by the Bureau of Motor Carrier Safety of the Department of Transportation, they attributed $38.8 \%$ of the accidents to the driver either having fallen asleep, being inattentive, or improperly reacting 
to emergency situations. An extensive survey of commercial vehicle drivers indicated that $23 \%$ had "ever had an accident or a 'close call' after long hours of driving or when they were tired" (Harris et al. 1972, p. 61-62). The oklahoma Turnpike Authority (1956) reported that 258 of the accidents it evaluated were caused by the driver having fallen asleep, while $38 \%$ of the fatal accidents were attributed to this cause. They later updated these figures (cited in case and Hulbert, 1970) attributing $22 \%$ of all the accidents and $48 \%$ of the fatal accidents between 1953 and 1964 to the driver having fallen asleep at the wheel. While Baker (1967) stated that "drowsiness" accounted for only 9.88 of single-car accidents, she also reported (1967) that at least $24.1 \%$ of the contributing factors in the singlevehicle accidents investigated were due to "driver asleep" (p.36). Other studies failed to indicate that fatigue directly played such a large role in highway accidents. Treat, Tumbas, McDonald, Shinar, Hume, Mayer, Stansfer and Castellan (1979), for example, reported that fatigue caused only . 3 to $1.7 \%$ of accidents while "critical nonperformance errors", defined as "blackouts or dozing", were considered direct causes in 1.7 to $2.1 \%$ of the accidents investigated ( $\mathrm{p} .10)$. However, Treat et al. (1979) do point out that fatigue and

"non-critical performance errors" were implicated in many accidents that were attributed to other causes such as inattention.

These statistics tend to suggest that the driver who has fallen asleep at the wheel faces a large clear-cut risk of becoming seriously and often fatally injured. Drowsy or fatigued drivers may not appear as likely, at first glance, to become involved in such serious accidents, but the magnitude of their risk becomes more apparent when the extent of the contributing role of fatigue to other more direct causes is considered. Because fatigue has a more subtle impairing effect on highway accidents than does falling asleep, most studies have concentrated on this latter cause while neglecting to assess the impact of fatigue on accidents.

Like alcohol, the effect of fatigue on highway accidents also appears to range according to both the age and sex of the driver. The oklahoma Turnpike Authority (1956) reported that one-half of all the drivers who fell asleep at the wheel were less than 24 years of age, while $78 \%$ were below 34 years of age (the average age for arivers in other accidents). Harris et al. (1972), however, indicated that drivers over 45 years of age tend to be more susceptible to fatigue after driving for a shorter period of time than young drivers. Baker (1967) revealed that $24.5 \%$ of "contributing accident factors" due to "driver asleep" were caused by males while $20.9 \%$ were caused by females ( $p$. 39).

There is also a slight suggestion that driver fatigue may involve distinct types of accident risks. In a survey (Harris et al. 1972) of commercial vehicle drivers involved in an accident or a "close call" after long hours of driving or when tired, drivers were asked to define the circumstances of the situation. While no particular circumstance was much more frequently described, $5 \%$ of the 
drivers did reply that a car had pulled out in front of them. In contrast, $4 \%$ indicated that they had been dozing and were running off the road." "Although the percentage of both accident types are low, and probably have little meaning in an absolute sense, they do tend to reflect two fundamentally different types of consequences that may be associated with fatigue at the wheel." (p. 62) The former circumstance involved a lack of alertness and hence an impaired response to another ariver's actions while the latter circumstance involves the fatigued driver alone (p. 61-62).

\section{3 Estimates of Alcohol and Fatigue Interactions}

Although individual accounts, intuition, and other indirect evidence strongly hint at the interacting role of alcohol and fatigue in highway accidents, their interaction has rarely been approached in any systematic way. Partly to blame for this is the insidious nature of fatigue. As previously discussed, because fatigue has not yet been clearly defined or measured in any objective and consistent manner, its effect on highway accidents becomes impossible to determine accurately. Fatigue's subtle and more indirect role in accidents may also be masked by attributing accidents to more direct causal factors. The example previously given was the situation where a legally intoxicated driver may have fallen asleep at the wheel and been fatally injured. Inspectors would easily detect his/her high BAC level but would be presented with little evidence to indicate that the driver had actually fallen asleep. Thus, given one clear-cut causal factor, the inspector may not have bothered to search for any other interacting variables. As Baker (1967) points out, many studies are set up such that the investigation of more than one causal factor is clearly discouraged. For example, inadequate time may be allotted for the investigation, the investigator may be inadequately trained, and the manner in which the investigation itself is conducted may be incomplete. In the study cited by Baker (1967), only 16.5\% of the accidents reviewed were attributed to more than one causal factor.

Because inspectors can, at best, only infer from the driver whether he or she has, in fact, been intoxicated or fatigued, the inspector is dependent on the driver for an explanation of the accident. Infortunately, it has been shown that while the inspector will rarely challenge the driver's explanation, the driver often provides a false account of the accident. In a study cited by Baker (1967), the investigator disagreed with the driver on the cause of the accident in only $6.3 \%$ of the cases (p. 42). The most frequent of these disagreements were those where alcohol or sleep was considered a factor by the police. Inspectors also felt that only $5.4 \%$ of the drivers gave false explanations regarding the cause of the accident when they were actually due to the driver having fallen asleep ( $p .37$ ). However, as Baker (1967) explains, drivers often have a large incentive for providing a false account of the accident: 
...drivers tend to explain accidents by which they have least culpability compatible with credulity. Drivers may sometimes tell investigators that they fell asleep when actually they were intoxicated. Driving under the influence is illegal and therefore more culpable than falling asleep while driving, which is not specifically unlawful. Likewise, when a driver falls alseep, he may be happy to explain the accident by some road or vehicle condition. ( $p .36$ )

The reported number of accidents in which drivers of different ages have been intoxicated or have fallen asleep is also subject to suspicion. "For young drivers, alcohol is conspicuously low and sleep is high. One wonders whether the opinions on which these curves are based represent reality or whether young drivers who have been drinking successfully explain their accidents to investigators by claiming to have fallen asleep--and if this is true for young drivers, could it not be true throughout the whole range of ages?" (Baker, 1967, p. 40). Whereas, one-half of all drivers who reported falling asleep at the wheel were below 24 years of age (Oklahoma Turnpike Authority, 1956), youngest drivers were (i.e., below 20) less frequently found intoxicated while driving (Jones and Joscelyn, 1978).

In spite of, or perhaps because of, these obstacles, only one study was found that directly attempted to assess the combined role of alcohol and fatigue. Although only $16.5 \%$ of the accidents investigated were attributed to combined factors, among these, the interaction of sleep and alcohol was (most frequently) implicated as a cause, making up 2.718 of the total number of accidents (Baker, 1967 ).

Through an analysis of their accident data, Treat et al. (1979) also suggest an interaction between alcohol and fatigue. They reported that alcohol intoxication may increase "by a factor greater than 5" the probability that critical nonperformance (defined as dozing, falling asleep at the wheel, or blacking out) may cause an accident ( $p .103$ ). They also suggest that alcohol may increase by a factor of two to three the likelihood that speeding, overcompensation and being internally distracted--performance decrements associated with fatigue--will have a causal involvement in accidents ( $\mathrm{p} .103$ ).

Accident frequency data for different times of the day further suggest the possibility of an interaction between those accidents attributed to fatigue or sleep and those attributed to alcohol intoxication. It has been found that both fatigue-involved and alcoholinvolved accidents occur much more freqently during the late evening hours, "the time of day or night when the highways are most lightly travelied [and] yet a proportionately larger number of fatal accidents occur...than during the hours of 6 to $11 \mathrm{a} . \mathrm{m}$. , when most persons are going to work, making business calls or going shopping". (Carolina Highways, 1953, p. 36). For example, $75 \%$ of the drivers killed in a accident between midnight and $6 \mathrm{a} \cdot \mathrm{m}$. were legally intoxicated, according to a Wayne County Study (Filkins et al., 1970). Similarly, the Department of Transportation Fatal Accident Reporting system 
(FARS) Annual Report (1978) indicated that $40 \%$ of the fatal single vehicle accidents in which the driver was legally intoxicated occurred between midnight and 3:59 a.m. These hours coincide with the hours in which most fatigue-related accidents occured. It has been reported that 50-568 of accidents due to falling asleep occurred between midnight and $8 \mathrm{a} \cdot \mathrm{m}$. (versus $11 \%$ between $8 \mathrm{a} \cdot \mathrm{m}$. and midnight) (McCoy, 1974; Oklahoma Turnpike Authority, 1956). Both alcohol- and fatiguerelated accidents also occur more frequently on weekend days (FARS Annual Report, 1978; Baker, 1967).

While it seems apparent that the frequency of alcohol- and fatigue-related accidents at different times of the day and day of the week are quite similar, the nature of this relationship remains unclear. To account for this relationship, each of three different explanations may apply. First, it may be argued that the conditions of nighttime driving are generally more conducive to accidents, regardless of cause. The FARS Annual Report (1978), for example, stated that $67 \%$ of all fatal accidents occured between $4 \mathrm{p} \cdot \mathrm{m}$. and $3: 59$ a.m., as opposed to only 338 between $4 \mathrm{a} \cdot \mathrm{m}$. and $3: 59 \mathrm{p} \cdot \mathrm{m}$. A second stance contends that the high percentage of alcohol-related accidents in the nighttime merely reflects the larger number of intoxicated drivers during the night than during the daytime hours. Supporting evidence of this comes from Farris et al. (1976) who found that the percentage of drinking drivers not involved in accidents--20 to $40 \%$ of all drivers-is also much higher in the late nighttime. Zylman (1973) has even reported that with a BAC of $0.50 \%$, the likelihood of an accident is actually three times greater between $9 \mathrm{a} \cdot \mathrm{m}$. and noon than it is between $3 \mathrm{a} \cdot \mathrm{m}$. to $6 \mathrm{a} \cdot \mathrm{m}$.

It may also be argued, however, that there is indeed an interaction between the causal involvement of alcohol and fatigue in highway accidents. Within this interaction, again, there is room for interpretation. The depressing effect of alcohol may act upon the intoxicated ariver to induce fatigue. It is also possible that fatigue, resulting from either having driven a long time, or from previous conditions (such as working all day and/or performing strenuous activities) may interact with the impairment associated with the intoxication. Also, the diurnal pattern of arousal may interact with alcohol to enhance impairment during night-time hours. The most probable explanation would account for all of these factors.

In summary, while the evidence suggests that alcohol and fatigue do interact in their effect on highway accidents, the extent and nature of this interaction cannot yet be adequately determined. 


\subsection{POSSIBLE REMEDIES TO COUNTERACT FATIGUE-RELATED DRIVING}

IMPA I RMENTS

Much attention and money has been invested in research on development, testing, and implementation of alcohol countermeasures and programs. These include community projects, public information and education campaigns, rehabilitation programs, legal remedies and enforcement procedures, and instrumentation and interlock system development (see Driessen \& Bryk, 1973, for a review and list of 107 alcohol countermeasures that have been suggested). Much less effort has been directed toward developing fatigue countermeasures or remedies. However, some proposals have been advanced and will be discussed in this chapter.

The most obvious fatigue remedy is rest. Although intuitively one would expect a rest break to aid in the recovery of a driver from fatigue effects, Harris et al. (1972) report data which indicate that rest break efficacy diminishes as a function of hours of trip time. The first rest break, occurring typically after about three hours of driving, is followed by evidence of both reduced driver error and physiological recovery. A second break, around six hours, was not as obviously beneficial as the prior one and had no effect whatever on the tendency of increased driver errors. The third rest break, coming at about nine hours into the trip, produced no recovery at all, and, in fact, may have contributed to a further decline in arousal subsequent to it.

Lewis (1978) suggests that a 15-hour driving limit be placed on individuals traveling intra-/interstate by the Department of Transportation. In addition, he states: "At least every seventy-five miles, a driver should stop (religiously), check the equipment, and more importantly, walk around and rejuvenate himself" (p. Il).

A second class of remedies involves distraction or intermittent stimulation. In vigilance studies, any disinhibition when coupled with performance errors counteracted performance decrements (see the use of shock by Dureman and Boden, 1972). Distraction and stress, while disturbing driving behavior under most conditions, nonetheless appear to alleviate the phenomenon known as "highway hypnosis" (Shor \& Thackray, 1970). A wealth of research has shown that stress and distraction serve to increase mental fatigue in a prolonged task, but occasional mild stress and novelty help to restore efficiency, albeit temporarily (Broadbent, 1953; Mackworth, 1950; Wilkinson, 1960). Corcoran (1962) peformed work investigating the arousing effects of noise upon sleep-deprived subjects. Ninety decibels of white noise, presented continuously, was shown to be effective in reducing the deterioration in performance which usually characterizes sleep loss.

As is commonly known, highway engineers build mild stress and perceptual novelty into their highways to defend against drowsiness (e.g., highway road dividing-line raised bumps, the intermittent spacing of traffic signs, etc.). Textured median strips, shoulders 
and interlane boundaries can be used to provide tactile and/or auditory cues as to position errors, and, consequently, could be used to alert a fatigued driver before he/she runs off the road.

Other distractions have also been suggested to combat fatigue. Brown (1965) investigated the hypothesis that a car radio would have an alerting effect upon the ariver, though this effect was not demonstrated quite well enough in the study.

Endou, Oshima, Watanabe and Shingo (1979) suggest a rather simple solution to fatigue-while-driving effects. They state that chewing gum acts as "a palliative against drowsiness, although the only safe method of preventing accidents resulting from drowsiness at the wheel is to stop driving when fatigued" (p. 758). Harris (1967, cited in Hulbert, 1972) presents a brief review of countermeasures for driving fatigue which lend themselves to easy implementation:

Drivers have been faced with the problem of drowsiness for a long time and apparently no 'solution' has been found. Some methods for the alleviation of drowsiness that have been suggested are: singing, chewing a pack of gum, taking off the right shoe, and sitting on something hard. (p. 292)

Along another vein, a self-contained system consisting of a horn alarm, a battery and a pedal similar to an accelerator pedal has been designed to counteract fatigue (Williams, 1966, cited in Hulbert, 1972). The device, which is dependent on the driver for activation, requires the driver, when cognizant of the onset of fatigue or drowsiness, to depress the pedal and keep it fully depressed; failure to do so sets into motion the alarm system. Williams holds that when drowsy, the driver will naturally reduce pressure on the pedal, thereby sounding the horn.

Frederick ( 1966 ) developed what was termed the Alert-o-matic. A sequence of three driver reaction-eliciting events of an increasingly compelling nature make up the Alert-o-matic system. Upon activation, a light, which is on a cycle of 60 seconds off, five seconds illuminated, flashes on. It is turned off if the driver taps his horn. If he fails to notice the light, the car horn is then sounded for a period of three seconds. If the driver does not attend to the horn, the ignition is intermittently turned off and on for five seconds, whereupon, should the driver fail in this as well, the ignition is turned off completely.

Delco Radio (1961) designed a sophisticated highway-to-car alerting system whereby impaired drivers would receive responsecontingent signals when driving through designated stretches on a road. The system consists of radio transmitters which emit a coded voice signal when a car enters the "trigger zone". A receiver in the car is sensitized to the signal, and during the time the car is in the 
"trigger zone" (approximately six seconds at 65 miles per hour), the message is repeated. Failure to respond to the signal would initiate a series of arousing signal stimuli to alert the inattentive driver.

O'Hanlon (1971) has suggested the use of an alertness indicator based on heart rate variability. Previous research had shown that heart rate variability tended to increase with driving time and to be correlated with other factors influencing alertness. However, the correlation was far from perfect, and o'Hanlon recognized the need for further refinement of the measurements or the inclusion of concurrent measurements of other physiological variables.

A similar alertness indicator could be based on some performance. measure that changes with fatigue. One possible measure is steering wheel reversals, a measure that increases with fatigue, though again not in an exact correlation. Such a device could measure the number of steering reversals made by a driver during a set time unit and compare each measurement to a criterion. Measurements falling below the criterion would trigger an alarm. One version of an alarm based on steering reversals was built by Life Technology, Inc., of California (cited in O'Hanlon, 1971, p.8).

The efficacy of an alerting alarm when performance deteriorates beyond a certain point is supported by research on the effects of feedback on performance under conditions of fatigue. The effects of lack of sleep were shown to be mitigated by knowledge of results. That is, either complete or minimal feedback concerning performance enables the subject to somewhat offset the impairment brought about by sleep deprivation (Wilkinson, 1961).

Most of the alertness indicators described could also be developed as interlock systems that prevent operation of the car if the potential driver can not meet a set performance criterion, or alternatively would alert passersby that the vehicle operator is impaired. Attwood (1975) feels an interlock system that would be a viable alcohol countermeasure could be developed if:

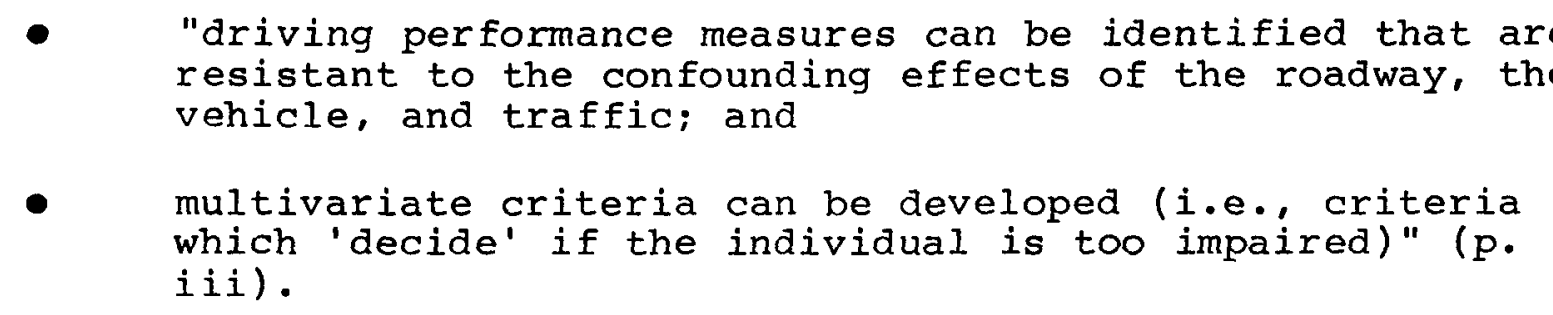

The same requirements apply to any potential fatigue interlock. Attwood (personal communication, 1981) has found that lane position variability best meets the above criteria. However, the equipment necessary to monitor lane position is very expensive and, as a consequence, is not practical for widespread use at this time. 
The final class of possible remedies for fatigue is stimulant drugs, primarily caffeine and amphetamines. Advocating the use of these drugs to counteract fatigue must take into account both the magnitude of the impairment caused by fatigue and the possible adverse effects of the drugs themselves. In addition, the interaction of these drugs and alcohol must be evaluated to determine whether their effect on fatigue is modified by the presence of alcohol.

Kleitman (1963) states that, while not knowing its exact method of aiding wakefulness, caffeine is an effective "anti-hypnotic agent". He also notes that caffeine ingestion produced faster reaction times and facilitated performance on muscular strength and speed of movement tasks.

Franks, Hagedorn, Hensley, and Starmer (1975) administered either alcohol $(75 \mathrm{mg} / \mathrm{kg})$ and caffeine $(300 \mathrm{mg} / \mathrm{kg})$, or caffeine alone. They found that the peak plasma alcohol concentration of about $92 \mathrm{mg} \%$ was not affected by caffeine. Performance decrements on a computer reaction time task induced by alcohol were antagonized by caffeine. The authors were unable to establish a clear pattern of alcohol antagonism using the doses of caffeine in the present study. Though drivers, subsequent to drinking a fair amount of alcohol, may feel more confident regarding their performance (Goldberg, 1943; McClelland, David, Kalin \& Wanner, 1972; Wallgren \& Barry, 1970), and more alert after taking in caffeine, the results indicate that actual performance is not improved to a significant extent; ordinary levels of caffeine do nothing to mitigate alcohol-induced impairment. As Nash (1966) points out, the ingestion of such stimulant drugs as caffeine may exacerbate the drinking and driving problem by bolstering motivation and capability for driving without thoroughly counteracting sensorimotor and judgment deficits.

Collins (1977) deprived subjects of sleep for 34 hours and 55 hours. Subsequent to the 55 hours of sleep deprivation, subjects were given $10 \mathrm{mg}$ of d-amphetamine. The results indicate that there were a larger number of errors (on a tracking task) for the 34-hour group than the controls; similarly, the 55-hour group exhibited more errors than the 34-hour group. The ingestion of the d-amphetamine produced a sharp drop in errors for the 55-hour group in tracking under static and dynamic conditions but it did not return performance levels to that of the control group and had less of an effect on tracking while moving (dynamic condition).

Hartmann, Orzack, and Branconnier (1977) compared the efferit: of d- and l-amphetamine on vigilance performance, waking EEG's and mood subsequent to one night of sleep deprivation. The results indicate that d-amphetamine was more powerful than 1-amphetamine in attenuating sleep deprivation deficits in vigilance and on waking EEG.

Rutenfranz and Jansen (1959) administered either $0.5 \mathrm{~g}$ or $1.0 \mathrm{~g} / \mathrm{kg}$ of alcohol to subjects who then ingested either $0.2 \mathrm{~g}$ of caffeine or $9 \mathrm{mg}$ methamphetamine. The results indicated that whereas 
both doses of alcohol impaired performance, a reversal of the low-dose effects and a partial reversal of the high-dose effects was accomplished by methamphetamine. It is interesting to note that the subjects did not report feeling the effects of alcohol, even though their blood alcohol concentration was $0.06 \%$. Caffeine was shown to have also reversed the deterioration of performance, but this effect was not as potent.

Wilson, Taylor, Nash, and Camerson (1966) investigated the interaction of alcohol $(1.2 \mathrm{~g} / \mathrm{kg}$; $\mathrm{BAC}$ 's ranging from 0.06 to $0.08 \%$ as measured by a breathalyzer) and amphetamine (15mg/kg), as well as an alcohol lactose dose (used as placebo). The performances of interest included pursuit rotor balance and skipping tasks, the Purdue peg board, Minnesota manipulation task, the Madsley Personality Inventory and a digit span test. The results indicated that there was no difference between the alcohol-amphetamine and alcohol-lactose conditions, perhaps because the tests were too insensitive to detect the subtle effects.

Alkuna (1977), after having administered alcohol doses of . $8 \mathrm{~g}$ of absolute ethanol per $\mathrm{kg}$ of body weight to subjects, administered doses of $1.5 \mathrm{~g}$ of either L-Dopa or placebo. It was found that L-Dopa lessened the alcohol-induced coordination and sensorimotor decrements. It was also shown that L-Dopa offsets slowing of the alpha rhythm caused by alcohol, indicating a possible facilitative effect upon central nervous system arousal. The authors also report that "BAC's taken 25, 50, and 110 minutes after the administration of L-Dopa were significantly lower than those for the placebo sessions suggesting further L-Dopa mitigative effects".

In summary, while many possible remedies have been suggested and tested, it appears that more research and development is required before these countermeasures can be implemented one a larger scale, as may be necessary. 


\subsection{SUMMARY AND CONCLUSIONS}

This chapter will summarize the major findings presented in this report on the effects of fatigue and alcohol on highway safety and assess the present state of knowledge of this area. A major impetus for this study is the suspicion that fatigue may be a factor in many so-called alcohol-related highway accidents. Thus, the summary will emphasize and speculate on research findings which are relevant to this concern.

Before beginning the summary, it is advisable to review some of the problems inherent in research on driving impairment and highway safety. Driving is a complex, coordinated psychomotor task. Many techniques have been employed to measure driving performance, ranging from on-the-road driving to laboratory tasks thought to be representative of some part of driving. The validity of laboratory tasks is often questionable, but experimental control can be good. Actual driving, in contrast, offers realism, and hence face validity, but does not permit good control over external variables. Thus, all techniques have some drawbacks. Furthermore, it is very difficult to determine what performance impairments are actually necessary to conclude that highway safety will be compromised.

In addition, the effects of alcohol and fatigue on driving performance or on any type of performance are often different for different individuals and under different conditions. Age, sex, amount of driving experience, personality, as well as a host of other factors, make it difficult to find consistent results and to draw general conclusions. Nevertheless, this chapeter will summarize the work that has been done and the general trends that have been found.

\subsection{Measures of Fatigue Which are Relevant to Driving}

The present state of our knowledge about the causes, mechanisms and effects of fatigue is still very primitive. There is no generally-accepted definition of fatigue, nor is there one technique for measuring fatigue that is widely accepted. A number of factors which lead to subjective feelings of fatigue and decrements in certain types of performances have been identified, but the relationships between input factors, subjective feelings, and objective performance changes differ among experiments and even among people in the same experiment. Furthermore, the subjective and objective changes are not always the same for all input factors.

Because there is no generally accepted definition or measure of fatigue, research on the effects of fatigue on driving performance must examine several measurement techniques. Fatigue can be operationally defined in two distinct ways:

by specifying the input factors which bring about fatigue, and 
- by measuring changes which occur when fatigue is present.

The input factors which have been found to be most likely to cause impairments in driving performance or performance related to driving are:

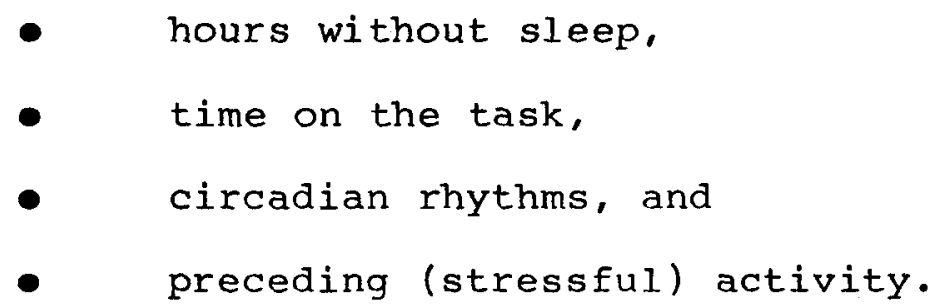

Sleep deprivation (hours without sleep) has been found to impair detection performance on visual (Wilkinson, 1960) and auditory (Deaton, Tobias \& Wilkinson, 1971) vigilance tasks, target detection tasks (Drucker, Cannon and Ware, 1969), visual search patterns (Kaluger \& Smith, 1970), information processing tasks (Bugge, Opstad \& Magnus, 1979), five-choice serial reaction time (RT) tasks (Wilkinson, 1961), tracking performance (Drucker, Cannon \& Ware, 1969), and vehicle control use measures (Forbes et al., 1958).

Many performance measures show decrements with increasing time on the task, usually driving. Some of these measures include: critical flicker fusion (Yajima, Ikena \& Oshima, 1976), some physiological measures of arousal (Harris \& Mackie, 1977; O'Hanlon \& Kel1y, 1977; Riemersma et al., 1977), vigilance performance (Mackworth, 1970), choice RT tasks (Romansky, 1978), tracking performance (Mast, Jones and Heimstra, 1965), and vehicle control measures such as velocity maintenance (Riemersma et al., 1977), steering wheel reversal rate (Sugarman \& Cozad, 1972) and lane position (Riemersma et al., 1977; Sugarman \& Cozad, 1972).

In addition, to considering time on the task, the type of task and conditions in which it is performed must be considered. Boring, monotonous conditions have a particularly deleterious effect on the ability to maintain alertness over time. The physiological correlates of arousal are higher driving on in-town roads than on rural roads (Riemersma et al., 1977). Vigilance performance shows less decrement over time if the signals occur frequently and are complex than if they are less frequent or less complex (Mackworth, 1970). Also, stressful conditions tend to cause a greater decrement over time than nonstressful conditions (Heimstra, 1970; Heimstra, Jones \& Dekock, 1965; Romansky, 1978).

Circadian rhythms also result in some changes in performance. Critical flicker fusion (Yajima et al., 1976), heart rate (Harris \& Mackie, 1977), auditory tone detection (Blake, 1967), and simple and choice reaction time (Kleitman, Titelbaum \& Feiveson, 1938) al1 follow a 24-hour cycle of increases and decreases. If performance is 
tested over more than one 24-hour cycle, there is often a circadian pattern superimposed on a general decrease over time (Bugge et al., 1979; Yajima et al., 1976).

Preceding activity either of a mentally fatiging or stressful nature can also cause performance impairments (Heimstra, Mast \& Larabee, 1963; Platt \& Fedderson, 1964).

In conclusion, all four of these fatigue factors must be considered in research on fatigue and driving. These four factors include the fatigue that arises from driving as well as the effect of fatigue from other sources on driving (Crawford, 1961).

The second way fatigue can be operationally defined is by measuring changes which result from a condition which is thought to be fatiguing. Three types of measures are useful in attempting to quantity fatigue incuding:

- physiological changes,
- subjective reports, and
- objective performance decrements.

Although the physiological changes which underlie or cause fatigue are not well understood, some physiological changes often accompany subjective feelings of fatigue. The most reliable include: decrease in heart rate and increase in heart rate variability (Harris \& Mackie, 1972; Riemersma et al., 1977; Sugarman \& Cozad, 1974), decrease in critical flicker fusion (Grandjean et al., 1977; Yajima et a1., 1976), decrease in electroencaphelogram frequency resulting in increasing alpha activity (Caille \& Bazanno, 1977; O'Hanlon \& Kelly, 1974) and slowing of the pupillary reflex to light (Lowenstein \& Lowenfield, 1952). However, the changes are not always continuous over time on a task or with increasing time without sleep. Thus, no one measure can be used as a reliable index of the amount of fatigue present. Furthermore, inter-individual variability necessitates recording of these measures in both alert and fatigued conditions and examining the differences. An index combining measures using multiple regression could potentially improve reliability over any one measure. One benefit of some physiological measures (e.g., heart rate, EEG, or skin conductance) is that they can be recorded continuously, and consequently, can show changes over time and in response to changes in task conditions.

One physiological change--pupillary dilation--has been found to correspond very closely with information processing demands. This measure appears to be one of the most promising physiological measures that could be used as a diagnostic technique with a short test (Yoss et al., 1970).

A number of subjective fatigue rating scales or questionnaires have been developed, and have proven to be fairly reliably correlated with performance decrements. Subjective reports have the advantage 
that they are independent of both the cause of fatigue and specific performance outcomes, so they can be used to compare the effects of different fatigue factors. However, since subjective scales rely on self-reports, they are always susceptible to false reports by any person wishing to influence his/her scale. Two of the most frequently used scales are the stanford sleepiness Scale and the Pearson-Byars Feeling-Tone Checklist. The Feeling-Tone Checklist, a 10-point internal scale, has been described as the best subjective scale presently available (Kinsman and Weiser, 1976).

The final measurement technique available is measurement of objective performance decrements. As Chapter 5 has shown, many types of performance are impaired by fatigue. However, the use of a performance test as a way to determine amount of fatigue seems unlikely. First, the only way performance can be evaluated is by comparison to a baseline measure for that individual in an alert state. Second, fatigue often results in irregularity of performance rather than an absolute decrement (Bills, 1931; Williams et al., 1959). Third, the effects of fatigue on a short performance test can be almost completely overridden by motivation (Wilkinson, 1961; williams et al., 1959).

Since the primary concern of research on fatigue and driving is with impairment in performance, performance measures are particularly important to investigate. Many measures of driving performance can be measured continuously, so they can be used to examine changes over time driving in different conditions. Since fatigue often leads to irregularity in performance, measures of variability in performance seem most promising. The results of past research on fatigue and driving has indicated that the most sensitive measures are variability in lane position, velocity control and steering. The most sensitive nondriving performance measures are those which require continuous responding over at least 10-15 minute periods and are demanding in information-processing load (e.g., pursuit tracking, serial choice RT tasks), or those that are low in information content but require continuous monitoring (vigilance tasks).

\subsection{Relationships Between the Impairing Effects of Alcohol and Fatigue}

An issue of major interest for highway safety is whether fatigue exacerbates the impairing effects of alcohol. If it does, then to the extent that fatigue and alcohol are present at the same time, safety risks will be higher for any given level of alcohol intake.

Very little research has examined the combined effect of alcohol and fatigue on driving or driving-related performance. The research that has been done indicates both synergistic and antagonistic effects, although antagonistic effects predominated. However, it 
is difficult to make conclusive statements about those findings because the studies differed in many ways. Futhermore, many types of performance relevant to driving have not been examined at all as a function of both alcohol and fatigue.

One important finding is that there are large individual differences in the way in which either alcohol or fatigue or their combination affect different individuals (e.g., Gibbs, 1968). A second general point to be made is that it is necessary to consider alcohol level in investigations of interactions. Low BAC's have been found to reduce the adverse impact of sleep loss on performance while high BAC's increased it (Wilkinson \& Colquhoun, 1968). Also, some effects showed interactions at moderate $B A C^{\prime} s$ when there was no interaction at low BAC's (Peeke et al., 1980).

The effects of alcohol alone and fatigue alone were also compared to find similarities. Although further investigations must be undertaken to confirm or deny interactions, similarities in performance decrements caused by alcohol and fatigue indicate possible areas in which additive or synergistic interactions may be found.

Both alcohol (at moderate and high levels) and fatigue have depressant effects on the central nervous system. Low levels of alcohol, in contrast, often act as a stimulant. Neurophysiological research suggests that the reticular activating system (RAS) is the site in the nervous system that is most susceptible to alcohol influences upon its functions (Kalant, 1970; Wallgren \& Barry, 1970; Williams \& Salamy, 1972). Since the RAS also plays a crucial róle in arousal and attention, some similarities between alcohol and fatigue effects on these processes might be expected. The direct role of the RAS in the regulation of arousal and attention indicates an area where physiological and behavioral changes due to the two factors might be similar.

A number of similarities between alcohol and fatigue effects are all relevant to a narrowing of attention. These include:

- decreases in information-processing capacity and rate,

reduced ability to process information from the peripheral retina, and

- changes in eye movement patterns.

Demanding information-processing tasks (including visual recognition, divided attention, serial choice reaction time tasks, letter-number substitutions, etc.) show decrements in either speed, accuracy or both as a result of alcohol (Allen et al., 1975, Buikhuisen \& Jongman, 1970; Hamilton \& Copeman, 1970; Moskowitz \& Roth, 1971; Moskowitz \& Sharma, 1974) and fatigue (Bills, 1931, 1935a, 1935b; Bugge et al., 1979; Schmidtke, 1965; Wilkinson, 1961; Williams et al., 1959). 
The use of peripheral vision is reduced both by alcohol (Buikhuisen \& Jongman, 1970; Hamilton \& Copeman, 1970; Huntley, 1970; Moskowitz \& Sharma, 1974; Von Wright \& Mikkonen, 1970) and fatigue (Mackworth, 1971; Weltman \& Egstrom, 1966) although the reduction is primarily found when there are other simultaneous information-processing demands. Thus, the visual field reductions appear to be the result of attentional limitations rather than sensory impairments.

Eye movement studies have found that scanning patterns are less active and more concentrated in central areas of the visual field as a result of alcohol (Beideman \& Stern, 1976; Belt, 1969; Moskowitz et al., 1976; Shroeder et al., 1974) and fatigue (Kaluger \& Smith, 1972; Rackoff \& Rockwell, 1975; Rockwell, 1972). Both alcohol (Beideman \& Stern, 1976; Belt, 1969; Buikhuisen \& Jongman, 1976; Shroeder et al., 1974 ) and fatigue (Beideman \& Stern 1977; Kaluger \& Smith, 1972) result in increased duration of fixations, indicating reduced processing rate. Also, both alcohol (Wallgren \& Barry, 1970) and fatigue (Caille \& Bassano, 1977; O'Hanlon \& Kelly, 1974) reduce the frequency of cortical activity as measured by EEG's (Peeke et al., 1980). Similarly, critical flicker fusion, which is often used as an indicator of central nervous system condition (as well as an indicator of temporal visual activity) decreases with both alcohol (Wallgren \& Barry, 1970) and fatigue (Grandjean, 1970; Yajima et al., 1976).

Some similarities between the effects of alcohol and fatigue are also found in overall vehicle control. Primarily, vehicle control measures show increased variability as a result of either factor. Velocity maintenance is generally impaired under alcohol (Attwood et al., 1980; Mortimer \& Sturgis, 1979; Huntley \& Centybear, 1974) and fatigue (Forbes et al., 1958; Platt \& Fedderson, 1964 Riemersma et al., 1977) but the changes due to fatigue are not consistently found. Lane position also becomes more variable with both alcohol (Allen et a1., 1975; Huntley \& Perrine, 1971; Sugarman et al., 1973) and fatigue (Caille \& Bassano, 1977; Dureman \& Boden, 1972; Mackie \& O'Hanlon, 1977; Riemersma et al., 1977; Sussman, \& Morris, 1970).

With both: alcohol and fatigue lateral position becomes more variable: however, the mechanism is somewhat different. Alcohol leads to an increase in control use, particularly steering wheel reversals (Huntley \& Centybear, 1974; Huntely, Kirk \& Perrine, 1972; Huntley \& Perrine, 1971), while fatigue often results in a decrease (Forbes et al., 1958; Greenshields, 1966; Sugarman \& Cozad, 1972). The variability in lateral position increases with fatigue because the size of the steering movements increases (Sugarman \& Cozad, 1972).

In conclusion, there are two main aspects of driving performance that are impaired similarly by alcohol and fatigue. They are: attentional capacity and vehicle speed and position control. 


\subsection{Estimates of the Proportion of Alcohol-Related Accidents}

that can be Attributed to Fatigue.

Most studies attribute 40 to $55 \%$ of fatal accidents on the road to alcohol intoxication (Filkins et al., 1970; Hurst, 1973).

An assessment of the role of fatigue in highway accidents is much more difficult because fatigue has been neither objectively. nor consistently defined, leading to different findings for different definitions. Also, there is currently no objective way to detect the presence of fatigue in the body as there is with alcohol. Furthermore, the contributory role of fatigue may often be neglected in accidents where other causal factors are more directly accountable, such as inattention or excessive speed. However, many direct causes such as "inattention", "improper lookout", "misperceptions", or other perceptual or judgment errors could themselves be the result of fatigue, in addition to the more clear-cut cases of "falling asleep at the wheel", "dozing", and "passing out".

Estimates of the involvement of fatigue in fatal accidents range from about 2\% (Treat et al., 1977) to 35-50\% (Forbes et al., 1957; Harris et al., 1972; Hulbert, 1972).

Evidence suggesting the interaction of alcohol and fatigue includes the following:

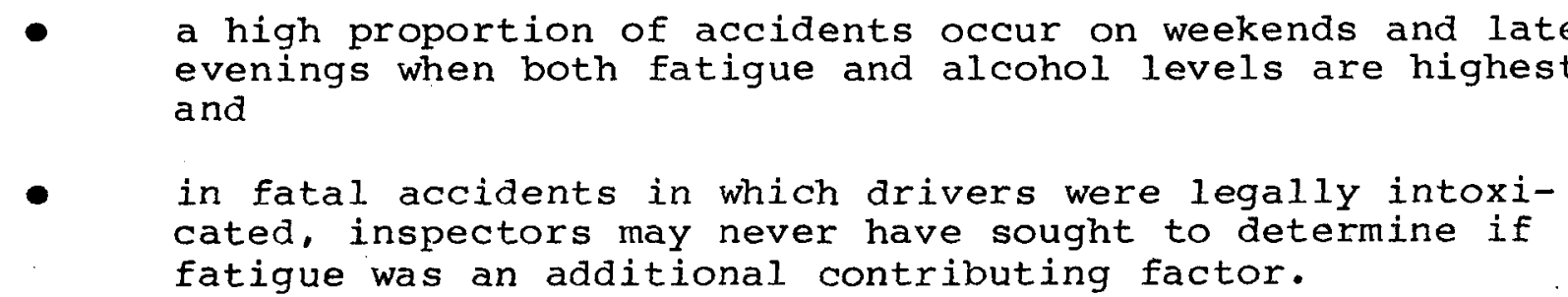

However, there are also arguments to the contrary including:

- alcohol ingestion may itself cause fatigue-like effects such as inattention, unresponsiveness or passing out, whether or not the individual was fatigued from some previous condition (Nelson et al., 1979); and

the fact that more accidents occur when both alcohol and fatigue levels are highest may be coincidental--there is no current evidence of causal relationships.

One study which attempted to assess the combined role of alcohol and fatigue (Baker, 1967) reported $16.5 \%$ of accidents investigated were attributed to combined factors, and of those $2.71 \%$ were attributed to alcohol and sleep. Also, Treat et al. (1979) reported that alcohol intoxication may increase by a factor greater than five the probability that falling asleep, dozing or passing out will cause an accident. 
Although the evidence strongly hints at the interacting role of alcohol and fatigue in highway accidents, their interaction has not been studied systematicaliy. Thus, there is not enough data available to determine what proportion of alcohol-related accidents could more properly be attributed to fatigue.

\subsection{Remedies That Have Been Suggested to Counteract Fatigue- Related Impairments}

Much less effort has been directed toward developing fatigue countermeasures or remedies than has gone toward alcohol countermeasures and programs. In spite of this a number of proposals have been advanced, some of which are easily implemented and others which require further development and testing. Three types of remedies have been suggested including:

- rest breaks,

- stimulant drugs, and

- intermittent or disinhibitory stimulation.

The most obvious fatigue remedy is rest. This type of remedy is one that any driver knows about and can implement easily if he/she wants to. Legal hours of driving limits can also be put into effect for commercial drivers.

A second class of remedies involves the use of stimulant drugs, primarily caffeine and amphetamines. Caffeine is widely available and useful to aid wakefulness. However, its use for alcohol and fatigue together is more questionable because caffeine does not mitigate alcohol-induced performance impairments (McClelland et al., 1972; Wallgren \& Barry, 1970) although it may make the individual feel more confident regarding his/her ability. While amphetamines also mitigate fatigue-related performance decrements (Collins, 1977), the possible adverse effects of these drugs limit their usefulness as a remedy.

A third class of remedies involves distraction or intermittent stimulation. Any disinhibition when coupled with performance errors can counteract performance decrements (Dureman \& Boden, 1972). Knowledge of the performance decrement can have the same effect (Wilkinson, 1961). One method of implementing this type of remedy is through the use of an alerting alarm activated by performance decrements or their correlates such as heart rate variability (O'Hanlon, 1971), number of steering wheel reversals, lane position variability, or some multivariate criteria. Measurements falling below some set criterion would trigger the alarm. Alternatively, an alerting signal which required a response to be turned off could be triggered intermittently (Delso Radio, 1961; Frederick, 1966). 
Another type of disinhibitory stimulation involves the use of textured median strips, shoulders, and interlane boundaries. These can provide cues as to position errors which alert the driver before he/she runs off the road. 


\subsection{RECOMMENDATIONS FOR FUTURE RESEARCH}

The literature reviewer in this project suggests that alcohol and possibly fatigue are major factors which impair driver performance and contribute to highway accidents. In addition, there is evidence suggesting that fatigue may exacerbate the impairing effects of alcohol. Thus, there is a need for further investigation of this problem. Suggestions for research related to each of the four objectives of this project are presented in the four subsections of this chapter.

\subsection{Development of Fatigue Measures}

Research on the effects of fatigue or the extent of its involvement in causing accidents will he successful only to the extent that fatigue can be measured in a reliable way. An ultimate goal of future research is to develop a quantifiable measure of fatigue similar to that used to quantify alcohol intoxication (blood alcohol concentration). That measure, to be valid, should bear a direct relationship with the resulting performance decrements. If that goal is achieved, it could be used to investigate the differences between different fatigue factors with regard to the amount of fatigue they cause. Furthermore, it could be used for research purposes without having to create the fatigue (operationally defined in terms of fatigue factors) as part of the research. A fatigue test could also be used in epidemiological work or accident investigations.

Subjective fatigue scales provide the most promising technique currently available. Further research is necessary to determine the validity of available instruments. Since subjective scales are based on self-reports, they require the cooperation of the individual being tested. Consequently, these measures may not be useful in epidemiological studies, accident investigations, or as an enforcement tool, because they can be subverted.

Developing a measure of fatigue that cannot be influenced by the individual being tested is a more difficult problem. Motivation can overide or at least mitigate fatigue effects on most short performance tests. Motivation or arousing events can also influence most physiological measures of fatigue. Changes in autonomic nervous system responses, however, may be less susceptible to motivation than central nervous system responses. In this regard, the work of Yoss (Yoss, 1969; Yoss et al., 1970) on pupil size fluctuations as a technique to diagnose sleepy drivers could be followed up.

11.2. Research to Determine the Relationship Between the Impairing Effects of Alcohol and Fatigue

More research is necessary to assess the nature and extent of this relationship. Very few studies have included both alcohol and fatigue, so present knowledge is based primarily on an evaluation of the similarities of the performance impairments caused by each alone. 
It is difficult to draw conclusions even from this work in many cases because of a wide variety of methodological problems. Following are some recommendations for the direction future research should take and some methodological considexations that should be taken into account in the planning of that research.

Examination of the effects of alcohol under conditions of fatigue. To determine how fatigue interacts with alcohol, and particularly if it exacerbates the impairing effects of alcohol, research on the joint effects of the two factors is necessary. To be informative about interactions, these studies should use orthogonal withinsubjects designs. That is, each subject should be tested under each condition, and the conditions used should include, at a minimum, alcohol alone, fatigue alone, both alcohol and fatigue, and a control condition in which neither is present. The orthogonal design is the only way to adequately examine the interactions of two independent variables. The within-subjects design is also advisable because of the large individual differences in susceptibility to alcohol and fatigue effects. Further experimental control can be obtained by varying the order of the conditions for each subject to control for practice effects over the sessions; and by using a double-blind procedure to preclude expectations of the experimenters from biasing the results of any session.

Investigations should be conductecd under the conditions of fatigue in which driving accidents usually occur. That is, the investigations should be conducted at night. At that time the number of hours without sleep is high (within the normal range), circadian rhythms are beginning to fall, and whatever stresses that occurred during the day have built up their accumulated effect.

Inclusion of low, moderate, and high alcohol dosages. Alcohol produces "bi-phasic" effects--low dosages often have a stimulating action, while moderate and high dosages have primarily depressant effects. Furthermore, in the studies of alcohol and fatigue interactions on performance that have been conducted, the interactions sometimes were different at low (BAC < $0.05 \%$ ) than at moderate (BAC > $0.05 \%$ and < $0.08 \%$ ) or high (BAC>0.08\%) dosages. In particular, the possibility that low levels of alcohol mitigate the impairing effect of fatigue while moderate and high levels exacerbate it must be considered. Alcohol levels just below the level of legal intoxication ( $B A C>0.08 \%$ and $<0.10 \%$ ) are important to examine. If fatigue exacerbates the impairment of alcohol, then the effects of an amount of alcohol which is legally permissible, when ingested under conditions of fatigue might produce as great or greater impairments than illegal levels of alcohol in an alert individual.

Use of closed-course driving tasks and interactive driving simulators. Closed-course driving tasks offer the best compromise between the realism and validity of actual driving and the experimental control of a laboratory situation. The coordination of the many simultaneous perceptual and motor skills that are required in actual driving are maintained in closed-course tasks. The same responses are 
required, and the same consequences of those responses occur as in onthe-road driving. Even the most sophisticated driving simulators AO not provide realistic sensory feedback of the effects of any particular control response (e.g., all the visual, vestibular, and proprioceptive changes that occur when the steering wheel is turned and the vehicle changes course). Also, an important element of realism that is always missing in the laboratory is the subject's knowledge that the consequences of driving control responses affect $\mathrm{his} / \mathrm{her}$ own safety. Motivational factors have been found to have a significant influence on the effects of both alcohol and fatigue. One aspect of driving that cannot be duplicated easily in closed course tasks is urban traffic conditions and the attentional demands of changing numbers of other vehicles and pedestrians.

A number of closed-course driving tasks which duplicate the demands of actual driving tasks have been used successfully to examine alcohol impairment and other impairments (Attwood et al., 1980). These include velocity-maintenance open-road driving, car-following with variable lead-car velocity, stopping, and two-lane passing. closed-course driving tasks of these types may provide sensitive tests of the interaction of alcohol and fatigue on performance.

Driving simulators, particularly interactive ones, provide a complementary method to closed-course driving. Driving simulators maintain a good deal of the realism of actual driving and allow a greater amount of experimental control than closed-course driving tasks. The same exact situation can be presented under different conditions to each subject. Also, complex driving situations involving other vehicles or obstacles can be presented to test specific perceptual and decision-making abilities.

Use of multivariate measures or test batteries. In many cases one variable has not been consistently related to alcohol or fatigue level. Since driving is composed of a number of interrelated behaviors, the use of multivariate measures may improve reliability in determining impairment. It may be possible for a driver to compensate for one impaired behavior with improved performance on another, but not to maintain all parts concurrently. Multivariate descriptors composed of vehicle control measures and/or physiological measures, which can be recorded continuously, have been used to discriminate between sober and drunk drivers when individual measures could not (Attwood et al., 1980). In that work and other research as well, measures of variability in performance were more sensitive to impairment than absolute change measures.

Control of confounding variables. Many variables influence the effect of either alcohol or fatigue on performance. These variables need to be taken into account in future research. Driver characteristics that need to be considered include: 


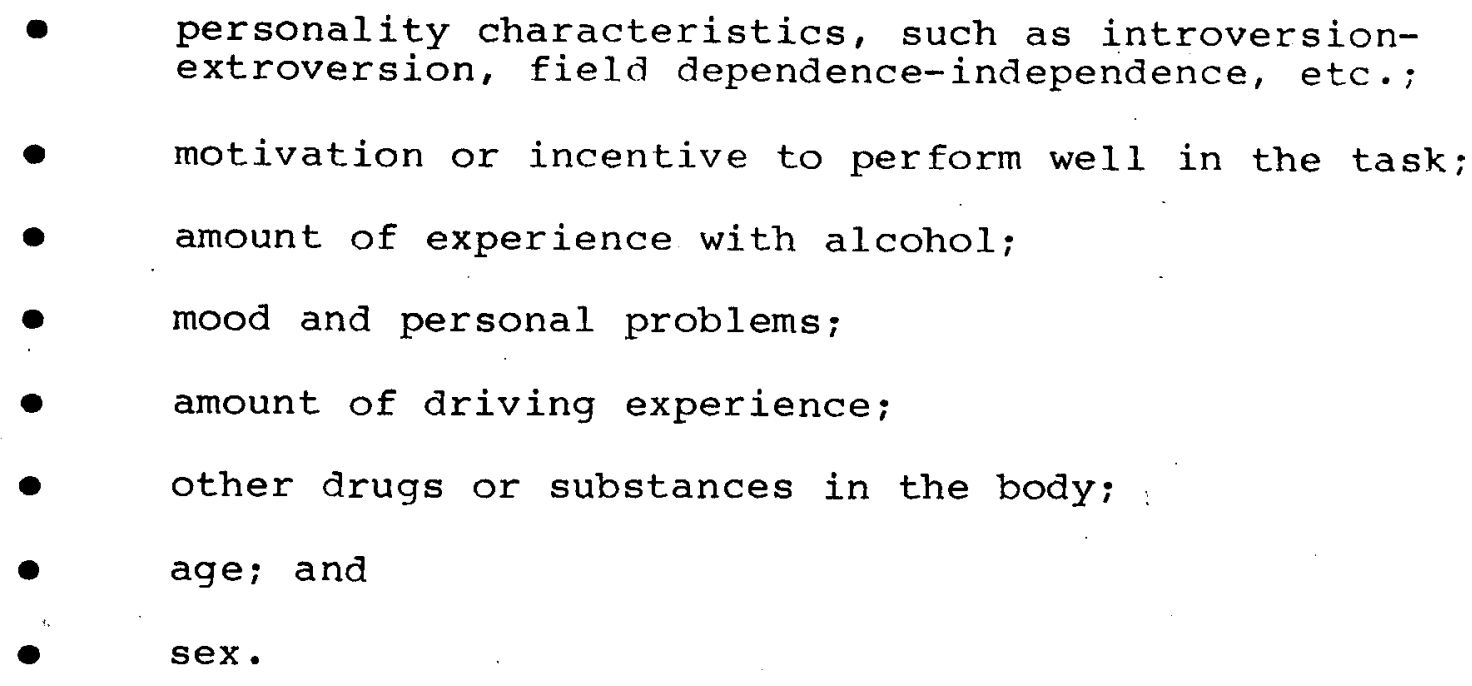

other environmental and vehicular characteristics which should be noted inlcude:
- light conditions--daylight, dusk, or dark;
- high or low demand driving conditions; and
- other environmental stressors such as noise or temperature extremes.

\subsection{Epidemiological Research to Determine the Proportion of Accidents that are Attributable to Alcohol, Fatigue, or a Combination}

Since there is not enough data available at the present time to determine the proportion of accidents which have been attributed to alcohol that are more appropriately attributed to alcohol and fatigue or to fatigue alone, further investigations are necessary.

Determining whether fatigue is involved in fatal accidents is difficult. There is no way known to test for the presence of fatigue in the body, as there is with alcohol or other drugs. Our present ability to detect fatigue depends on the cooperation of drivers. Thus, one possible approach might be a survey study in which drivers are stopped and asked about their current state, recent activities and the extent to which they drive when fatigued. The survey could also include questions about whether the person had been involved in any minor accidents or near accidents, and if so, what their cause was. Such a study should sample different types of driving environments (urban vs. rural), different times of the day and week, and different age drivers. 
11.4 Development and Testing of Remedies to counteract the Impairing Effects of Fatigue

Many simple remedies are available to anyone driving to counteract fatigue including: rest breaks, coffee, opening the window, or turning on the radio. Most people are aware of these remedies. They also are aware of the danger of falling asleep at the wheel and are able to tell when they feel tired. Therefore, public information and education campaigns, such as have been used with alcohol, do not appear to hold much promise of effectiveness with fatigue.

The possibility of legal remedies is also doubtful without a method for testing fatigue that is relatively accurate and not subvertible. Hours of service regulations for commercial drivers are already in effect and should be updated as new information becomes available.

The area in which further work developing countermeasures might he most productive is an in-vehicle alerting device. As was discussed in Chapter 8, an alerting device which is activated when performance falls below some set criterion could be effective in counteracting further performance decrements. What is necessary is research to determine what dependent measure or combination of measures, could be employed. The measure(s) need to be a valid indicator of driving ability or impairment yet must remain insensitive to the influence of vehicle and roadway factors. 


\section{$12.0 \quad$ REFERENCES}

Abele, G. Die Geschwindigkeisleitung des Kraftfahreus unter

Alkoholeinfuss. Deutsche Zeitschrift fuer die Gesampte Gerichtliche Medizin, 1958, 47, 447-461. (Cited in Wallgren and Barry, 1970).

Alkuna, R.L., et al. Reversal of ethanol intoxication in man: An assessment of the efficiency of 1 -dopa, aminophylline, and ephedrine. Psychopharmacology, 1977, 55, 203-209.

Allen, R.W., Jex, H.R., McRuer, D.T., \& DiMarco, R.J. Alcohol effects on driving behavior and performance in a car simulator. IEEE Transactions on Systems, Man and Cybernetics, 1975, SMC-5(5), 498-505.

Aschan, G., Bergstedt, M., Goldberg, L., \& Laurell, L. Positional nystagmas in man during and after alcohol intoxications. Quarterly Journal of Studies on Alcohol, 1956, 17, 381-405.

Asknes, E.G. Effect of small dosages of alcohol upon performance in a link trainer. Journal of Aviation Medicine, 1954, 25, 680-688, 693 .

Attwood, D.A. The potential of using driving performance measures in an alcohol interlock. Technocal memorandum 75/4, Mínistry of Transport, Canada, 1975.

Attwood, D.A. Personal communication, 1981.

Attwood, D., Williams, R.D., \& Madill, H.D. Effects of moderate blood alcohol concentrations on closed-course driving performance. Journal of Studies on Alcohol, 1980, 41, 623-634

Bahnsen, P., \& Vedel-Petersen, K. Alcohol studies: I. Experiments on drivers of motor vehicles. Journal of Industrial Hygiene, 1934 , $16,304-322$.

Baker, J.S. Single-vehicle Accidents on Rte. 66. Highway Research Record, 1967, 188, 26-43.

Bartlett, F.C. Psychological criteria of fatigue. In W.F. Floyd and A.T. Welford, (Eds.), Fatigue. London: H.K. Lewis, 1953.

Bartley, S.H. Fatigue: Mechanism and Management. Charles C. Thomas, Springfield, IIl, 1965.

Bartley, S.H., \& Simonson, E. Use of visual methods for measurement of general fatigue. In Simonson \& Weiser (Eds.), 1976.

Beatty, J., \& Wagoner, B.L. Pupillometric signs of brash activation vary with level of cognitive processing. Science, 1978, 199, $1216-1219$. 
Beideman, I.., \& Stern, J. Visual search activity and motor performance under alcohol intoxication. In M. Horvath (Ed.), Adverse Effects of Environmental Chemicals and Psychotropic Drugs (Vol. 2). New York: Elsevier, 1976 .

Beideman, L.R., \& Stern, J.A. Aspects of eye blink during simulated driving as a function of alcohol. Human Factors, 1977, 19, 73-78.

Belt, B. Driver eye movements as a function of low alcohol concentrations. Columbus, Ohio: Ohio state University, Dept. of Industrial Engineering, Driving Research Laboratory, June, 1969.

Bennett, R.M., Buss, A.H., \& Carpenter, J.A. Alcohol and human physical aggression. Quarterly Journal of Studies on A1cohol, 1969, 30, $870-876$.

Berger, C., \& Mahmeke. Fatigue in two simple visual tasks. The American Journal of Psychology, 1954, 67, 509-512.

Bernhard, C.G., \& Skaglund, C.K. Selective suppression with ethyl alcohol of inhibitions in the optic nerve and of the negative component PIII of the electroretinogram. Acta Physiologica Scandinavica, $1941, \underline{2}, 10-21$.

Bertera, J.H., \& Parsons. Impaired visual search in alcoholics. Alcoholism, 1978, 2, 9-14.

Bietti, G.B., \& Giardini, A. The effect of anoxia on eye movements. II. Comparative study of the action of anoxia and alcohol, separately and together. Riv. Med. Aeronaut, 1949, 12, 198-210.

Billings, C.E., \& Wick, R.L. Effects of alcohol on pilot performance during instrument flight. Federal Aviation Administration, Technical Report, FAA-AM-42-4, 74, 1972.

Bills, A.G. Blocking: A new principle of mental fatigue. American Journal of Psychology, 1931, 43, 230.

Bills, A.G. Fatigue, oscillation, and blocks. Journal of Experimental Psychology, 1935, 18, 562-573. (a)

Bills, A.G. Some causal factors in mental blocking. Journal of Experimental Psychology, 1935, 18, 170-185.

Bjerver, K. \& Goldberg, L. Effect of alcohol ingestion on driving ability. Quarterly Journal of Studies on Alcohol, 1950, 11, 1-30.

Blake, M.J.F. Time of day effects on performance in a range of tasks. Psychonomic science, $1967,9,349-350$.

Blum, B., Stern, M.H., \& Melville, K.I. A comparative evaluation of the actions of depressant and stimulant drugs on human performance. Psychopharmacologia, 1964, 6, 173-177. 
Roadle, J. Vigilance and simulated night Ariving. Ergonomics, 1976, $19,217-225$.

Bohne, G. Luff, K. \& Tratumann, H. Experimental studies on the possibility of compensating for disturbances of attention and motor functions caused by alcohol. Deutsche Zeitschrift Fuer die Gesamote Gerichtliche Medizin, 1957, 46, 226-234.

Borkenstein, R.F., Crowther, R.F., Shumate, R.P., Zeil, W.B., \& Zylman, $R$. The role of the drinking driver in traffic accidents. Bloomington, Indiana: Indiana University, Department of Police Administration, 1964 .

Boyd, E.S., Morken, D.A., \& Hodge, H.C. A psychomotor test to demonstrate a depressant action of alcohol. Quarterly Journal of Studies on Alcohol, $1962,23,34-39$.

Brecher, B.A., Hartman, A.P., \& Leonard, D.D. Effect of alcohol on binocular vision. American Journal of Ophthalmology, 1955, 39, 44-52.

Broadbent, D.E. Decision and Stress. London: Academic Press, 1971.

Broadbent, D.E. Neglect of the surroundings in relation to fatigue decrements in output. In W.F. Floyd \& A.T. Welford (Eds.), Symposium on Fatigue. London: Lewis, 1953.

Brown, I.D. Effect of a car radio on driving in traffic. Ergonomics, $1965, \underline{8} ; 475-479$ (a).

Brown, I.D. A comparison of two subsidiary tasks used to measure fatigue in car drivers. Ergonomics, 1965, 8, 467-473(b).

Brown, I.D. Decrement in skill observed after seven hours of car driving. Psychonomic science, 1967, 7, 1310132 .

Brown, I.D., Simmonds, D.C.V., \& Tickner, A.H. Measurement of control skills, vigilance, and performance on a subsidiary task during twelve hours of car driving. Ergonomics, 1967, 10, 665-673.

Brown, I.D., Tickner, A.H., \& Simmonds, D.C.V. Effects of prolonged driving upon driving skill and performance of a subsidiary task. Industrial Medicine and Surgery, 1966, 35, 760-765.

Brown, I.D., Tickner, A.H., \& Simmonds, D.C.V. Effect of prolonged driving on overtaking criteria. Ergonomics, 1970, 13, 239-242.

Buffard, s. Study of the psychomotor reactions of 22 subjects after ingestion of a moderate quantity of alcohol. Ann Med. Leg., 1959, $39,124-128$. 
Bugge, J.F., Opstad, P.K., \& Magnus, P.M. Changes in the circadian rhythm of performance and mood in healthy young men exposed to prolonged, heavy physical work, sleep deprivation and caloric deficit. Aviation, Space, and Environmental Medicine, 1979, 50, $663-668$.

Buikhuisen, W. , \& Jongman, R.W. Traffic perception under the influence of alcohol. Ouarterly Journal of studies on Alcohol, $1972,33,800-806$.

Bujas, Z., Sremec, B., \& Vidacek, s. Dozevljaj umora i njegove asocijacije s nekim drugim varij ablama. Archiv Hig Rada, 1965, 16, 111. (Cited in Simonson and Weiser, 1976).

Burg, A. The stability of driving record over time. Accident Analysis and Prevention, 1970, 2, 57-65.

Caille, E.J., \& Bassano, J.L. Validation of a behavior analysis methodology: Variation of vigilance in night driving as a function of the rate of carboxyhemoglobin. In R.R. Mackie, 1977 .

Carlson, A.J., Kleitman, N., Muehlberger, C.W., Mclean, F.C., Gullicksen, H., \& Carlson, R.B. Studies on the possible intoxicating action of 3.2 percent beer. Chicago: University of Chicago Press. 1934.

Carlson, W.L. Alcohol usage of the nighttime driver. Journal of Safety Research, 1972, $4,12-25$.

Carpenter, J.A. Effects of alcoholic beverages on skin conductance. ouarterly Journel of Studies on Alcohol, 1957, 18, 1-18.

Carpenter, J.A. Effects of alcohol on some psychological processes. Duarterly Journal of Studies on Alcohol, 1962, 23, 274-314.

Carpenter, J.A., \& Armenti, N.P. Some effects of ethanol on human sexual and aggressive behavior. In B. Kissen, and H. Begleiter, (Eds.) The Biology of Alcoholism. Vol 2. Physiology and Behavior., New York: Plenum Press, 1972.

Carpenter, J.A., Moore, O.K., Snyder, C.R., \& Lisansky, E.S. Alcohol and higher-order problem solving. Quarterly Journal of Studies on Alcohol, 1961, 22, 183-222.

Cass, L.J., \& Frederik, W.S. A new method of measuring the effects of drugs on performance. American Journal of Medical Science, 1961. $241,303-308$.

Chardon, G., Boiteau, H., \& Bogaert, E. Study of the action of weak doses of alcohol on central nervous system activity, Ann. Med. Leg.' $1959,39,462-472$. 
Chastain, J.D. The effects of 0.10 percent blood alcohol on ariving ability. Results of a practical experiment conducted at Austin, Texas on January 4 and 5, 1961. Austin, Texas Department of Furlic Safety, Identification and Criminal Records Division.

Chiles, W.D., \& Jennings, A.E. Effects of alcohol on complex performance. Report No. AM 69-14, Oklahoma City: Federal Aviation Administration, Office of Aviation Medicine, Civil Aeromedical Institute, 1969.

Clayton, A.B. Use of physiological measures in the assessment of driving load. In Psychological Aspects of Driver Behavior, Volume 1. Swov, The Netherlands: Institute for Road Safety Research, 1971.

Cohen, J., Dearnaley, E.J., \& Hansel, C.E.M. The risk taken in driving under the influence of alcohol. British Medical Journal, 1958, 1 , 1438-1442.

Coldwell, B.B., Penner, D.W., Smith, H.W., Lucas, G.H.W., Rodgers, R.F., \& Darroch, F. Effect of ingestion of distilled spirits on automobile driving skill. Quarterly Journal of Studies on Alcohol, 1958, 19, 590-616.

Collins, w. Some effects of sleep deprivation on tracking performance in static and dynamic environments. Journal of Applied Psychology, $1977,62,567-573$.

Collins, W., Schroeder, D., Gilson, R., \& Guedry, E. Effects of alcohol ingestion on tracking performance during angular acceleration. Journal of Applied Psychology, 1971, 55, 559-664.

Colquhoun, W.P. Biological Rhythms and Human Performance. New York: Academic Press, 1971.

Colquhoun, W.P., Folkhard, S., Knauth, P., \& Rutenfranz, J. Experimental Studies of Shiftwork. Oplander: Westdeutcher Verlag, 1975.

Colson, Z.W. The effect of alcohol on vision. An experimental investigation. Journal of the American Medical Association, 1940, $115,1525-1527$.

Coopersmith, S. The effects of alcohol on reactions to affective stimuli. Quarterly Journal of Studies on Alcohol, 1964, 25, 459-475.

Corcoran, D.W.J. Noise and loss of sleep. Quarterly Journal of Experimental Psychology, 1962, 14, 178-182.

Crancer, A., Dille, J.M., Delay, J.C., Wallace, J.E., \& Haykin, M.D. Comparison of the effects of marijuana and alcohol on simulated driving performance. Science, 1969, 164, 951-954. 
Crawford, A. Fatigue and driving. Ergonomics, 1961, 4, 143-154.

Cutter, H.S.G. Alcohol, drinking problems, and the psychological probability of success. Behavioral Science, 1969, 14, 19-27.

Cutter, H.S.G, Gree, L.R., \& Harford, T.C. Levels of risk taken by extraverted and introverted alcoholics as a function of drinking whiskey. British Journal of Social and Clinical Psychology, 1973, 12, 83-84.

Czeisler, C.A., Weitzman, E.D., Moore-Ede, N.C., Zimmerman, J.C., \& Knavrer, R.S. Human Sleep: Its duration and organization depend on its circadian phase. Science, 1980, 210, 1264-1267.

Damkot, K., \& Frysinger, R.C. Alcohol influence on hemispheric differences and signal detection thresholds. Psychopharmacology, 1978, 56, $173-177$.

Damkot, D.K., Toussle, S.R., Akley, N.R., Geller, H.A. \& Whitmore, D.G. On-the-road driving behavior and breath alcohol concentration. National Highway Traffic Safety Administration Technical Report DOT-HS-802-264, 1977.

Deaton M., Tobias, J.S., \& Wilkinson, R.T. The effect of sleep deprivation on signal detection parameters. Quarterly Journal of Experimental Psychology, 1971, 23, 449-452.

Delco Radio Highway Communicator. General Motors Corporation: Indiana, 1961 .

Docter, R.F., Naitob, P., \& Smith, J.C. Electro-encephalographic changes and vigilance behavior during experiemntally induced intoxication with alcoholic subjects. Psychosomatic Medicine, 1966, 28, 605-615.

Dott, B.A., \& McKelvey, R.K. Influence of ethyl alcohol in moderate levels on visual stimulus tracking. Human Factors, 1977, 19, $191-199$

Douglas, N.E., \& Johns, T.R. An investigation of the relationship between induced "fatigue" and heart rate. Chapel Hill, North Carolina: U.N.C., Highway Safety Research Center, 1973, 24.

Drew, G.C., Colquhoun, W.P., \& Long, H.A. Effect of small doses of alcohol on a skill resembling driving. British Medical Journal, $1958, \underline{2}$, 993-999.

Driver fatigue causes high percentage of accidents. Carolina Highways, 1953, 5,36 . 
Drucker, E.H., Cannon, L.D., \& Ware, J.R. The effects of sleep deprivation on performance over a 48-hour period. Human Resources Research office, 1969.

Dureman, E.I., \& Boden, C.H. Fatigue in simulated car driving. Ergonomics, 1972, 15, 299-308.

Ehlers, T. Alkoholbedingte motivation sarderunjeh und unfulluefahrdung. A Expt1. Angew. Psychol., 1966, 13, 1-18.

Ellingstad, V.S., \& Heimstra, N.W. Performance changes during the sustained operation of a complex psychomotor task. Erognomics, 1970 $13,693-705$.

Endou, T., Oshima, M., Watanabe, T.. \& Shingo, D. A preventive measure against drowsiness while driving. Ergonomics, 1979, 22, 762 .

Enzer, N., Simonson, E., \& Ballard, G. The effect of small doses of alcohol on the central nervous system. American Journal of Clinical Pathology, 1944, 14, 333-341.

Erwin, C.W., et al. Alcohol-induced drowsiness and vigilance performance. Journal of Studies on Alcohol, 1978, 39, 505-5í.

Evans, Martz, Rodda, Kiplinger, \& Forney. Clinical Pharmacology and Therapeutics, 1974, 15, 253-260.

Fagerstrom, K.O., \& Lisper, H.O. Effects of listening to car radio, experience and personality of the driver on subsidiary reaction time and heart rate in a long-term driving task. In R.R. Mackie, 1977.

Fagerstrom, K.O., \& Lisper, H.O. Sleepy drivers: analysis and therapy of seven cases. Accident Analysis and Prevention, 1978, 10, 241-250.

Farber, E. Passing behavior on public highways under daytime and nighttime conditions. Highway Research Record, 1969, 292, 11-23.

Farris, R., Malone, T.B., \& Lilliefors, H. A comparison of alcohol involvement in exposed and injured drivers. Phases I \& II. National Highway Traffic Safety Administration, Technical Report, DOT-HS-801-826, 1976.

Fatal Accident Reporting System. Department of Transportation, 1978 Annual Report.

Filkins, J.D., Clark, C.D., Rosenblatt, C.A., Carlson, W.L., Kerlan, M.W. \& Manson, H. Alcohol abuse and traffic safety: A study of fatalities, DWI offenders, alcoholics, and court-related treatment approaches. Final Report. U.S. Department of Transportation, National Highway Safety Bureau, Contract Nos. FH-11-6555 and $\mathrm{FH}-11-7129$, 1970 . 
Forbes, T.W., Katz, M.S., Cullen, J.W., \& Deterline, W.A. Sleep deprivation efects on components of driving behavior. Highway Research Abstracts, 1958, 28, 21-26.

Forney, R.B., Hughes, F.W., \& Greatbatch, W.H. Measurement of attentive motor performance after alcohol. Perceptual and Motor Skills, $1964,19,151-154$.

Franks, H.M., Haggedorn, H., Hersley, U.R., \& Starmer, G.A. The effects of caffeine on human performance, alone or in combination with ethanol. Psychopharmacologia, 1975, 45, 177-181.

Franks, H.M., Hensley, V.R., Hensley, W.J., Starmer, G.A., \& Teo, R.K.C. The relationship between alcohol dosage and performance decrement in humans. Journal of Studies on Alcohol, 1976, 37, 284-297.

Frederick, W.S. The alert-o-matic. Liberty Mutual Research Center. Hopkington, Massachusetts, 1966.

Fregly, A.R., Bergstedt, M., \& Graybiel, A. Relationships between blood alcohol, positional alcohol nystagmus and postural equilibrium. Quarterly Journal of Studies on Alcohol, 1967, 28, $11-21$.

Fruhstorter, H., et. al. Neurophysiological vigilance indicators and operational analysis of a train vigilance monitor device: A laboratory and field study. In R.R. Mackie, 1977.

Giardini, A. On the effects on the light sense of alcohol combined with anoxia. Revista Med. da Aeronautica (Rio de Janeiro), 1948, 11, 531-543. (Cited in Wallgren and Barry, 1970).

Giardini, A. The action of alcohol on the ability to fuse retinal images. Journal of Italian Ophthalmology, 1949, $2,446-451$.

Gibbs, C.B. The effect of alcohol and fatigue on decision processes and the precision of movements. 14th Conference, Canadian Highway Safety Council, Medical Session, May 6, 1968.

Goldberg, L. Quantitative studies on alcohol tolerance in man. The influence of ethyl alcohol on sensory, motor and psychological directions referred to blood alcohol in normal and habituated individuals. Acta Physiologica Scandinavica, 1943, 5, (Supplement, 16), 1-128.

Goldberg, L. Laboratory experiment with automobile drivers. Tirfirg, 1949, 43, 54-65.

Gordon, D.A., \& Mast, T.M. Drivers' judgements in overtaking and passing. Human Factors, 1970, 12, 341-346. 
Grandjean, E.P. Fatigue. American Industrial Hygenics Association Journal, 1970, 31, 1.

Greenberg, A., \& Carpenter, J.A. The effect of alcoholic beverages on skin conductance and emotional tension. Quarterly Journal of Studies on Alcohol, 1957, 18, 190-204.

Greenshields, B.D. Changes in driver performance with time in driving. Highway Research Record, 1966, 122, 75-88.

Gruner, O. Alkohol und aufmerk samkeit. Deutsch. z. ges. gerichtl. Med.. 1955, 44, 187-195. (Cited in Moskowitz, 1973.)

Gruner, 0 . Storungen der aufmerk samkeit bei neidrigen alkoholkonzentrationen. Hefte Unfalheilk, 1963, 77, 258-264. (cited in Moskowitz, 1973.)

Gruner, O., Ludwig, O., \& Domer, H. Zur abhanglgkeit alkohol bedingter aufmerksamkeitsstorungen vom blutalkoholvert bei neidrigen konzentrationen. Blutalkohol, 1964, 3,445-452. (Cited in Moskowitz, 1973.).

Gruner, O. \& Ptasnik, H. Zur Frage der Beeinflussung alkohol bedingten lei stingsabfalles durch Laevoilo-segaben. Mun. Med. Wach., 1953, 95, 931-933. (Cited in Walgren and Barry, 1970.)

Gruss, M., Berleiter, H., Tobin, M. \& Kissin, B. Changes in auditory evoked response induced by alcohol. Journal of Nervous Mental Disease, 1966, 143, 152-156.

Haddon, W.. Jr., \& Bradess, V.A. Alcohol in the single vehicle fatal accident. Journal of the American Medical Association, 1959, 169, 1587-1591.

Hamilton, P., \& Copeman, A. Effect of alcohol and noise on components of a tracking and monitoring task. British Journal of Psychology, 1970, 61, 149-156.

Harris, W., Mackie, R.R., Abrams, C., Buckner, D., Harabedian, A., O'Hanlon, J.F., \& Starks, J.R. A study of the relationships among fatigue, hours of service, and safety of operations of truck and bus drivers. (Technical Report 1727-2) Goleta, California: Human Factors Research, Inc., 1972.

Hartmann, E., Orzak, N.H., \& Branconnier, R. Sleep deprivation deficits and their reversal by $d-$ and 1 - amphetamine. Psychopharmacology, 1977, 53, 185-189.

Heimstra, N.W. The effects of 'stress fatigue' on performance in a simulated driving situation. Ergonomics, 1970, 13, 209-218. 
Heimstra, N.W., Mast, T.M. \& liarrahee, I.T. The effects of fatiaue on hasic psychological processes involved in human operator performance: Simple vigilance and Target detection. Dept. of Psychology University of South Dakota, 1963, Prepared for: South Dakota Dept. of Highways Research \& Planning Division and the U.S. Dept. of Commerce Bureau of Public Roads, Washington, D.C. pp. 1-19.

Herbert, M.J., \& Jaynes, W.E. Performance decrement in vehicle driving. Journal of Engineering Psychology, 1964, 3, 1-8.

Hess, E.H., \& Pott, J.M. Pupil size in relation to mental activity during simple problem-solving. Science, 1964, 143, 1190-1192.

Hetherington, E.M., \& Wray, W.P. Aggression, need for social approval, and human preferences. Journal of Abnormal and Social Psychology, $1964,68,685-684$.

Hoddes, E., Zarcone, V., Smythe, H., Phillips, R., \& Dement, W.C. Quantifications of sleepiness: A new approach. Psychophysiology, $1973,10,431-436$.

Holcomb, R.L. Alcohol in relation to traffic accidents. Journal of the American Medical Association, 1938, III, 1076-1085.

Honneger, H., Kampschulte, R., \& Klein, H. Alcohol disturbance of visual accuity for moving objects. Blutalkohol, 1970, I, $31-44$.

Hughes, F.W., \& Forney, R.B. Comparative effect of three anti-histimines and ehtanol on mental and motor performance. Clinical Pharmacology and Therapeutics, 1964, 5, 414-421.

Hulbert, s. Effect of driver fatigue. Human Factors in Highway Traffic Safety Research, 288-302, Wiley: N.Y., 1972.

Huntley, M.S. Effects of alcohol and fixation-task demands upon human reaction time to achromatic targets in the horizantal median of the visual field. (Doctoral dissertation, University of Vermont) Ann Arbor, MI: University Microfilms, 1970, 31, No. 3026-B.

Huntley, M.S., \& Centybear, T.M. Alcohol, sleep deprivation and driving speed effects upon control use during driving. Human Factors, 1974, 16, 19-28.

Huntley, M.S., \& Kirk, R.S. Influences of alcohol on emergency control-reaction times and brake pressure modulation during automobile driving. proceedings, sixteenth Annual Meeting of the Human Factors Society, Los Angeles, October, 1972.

Huntley, M.S., Jr., Kirk, R.S., \& Perrine, M.W. Effects of alcohol upon nocturnal driving behavior, reaction time, and heart rate. Paper presened at SAE Automotive Engineering Congress. Detroit, January, 1972. 
Huntley, M.S., \& Perrine, M.W. Influences of alcohol on driving behavior in an instrumented car.: Institute for Road Safety Research swov. Noordwigkerhout, The Netherlands, 1971.

Hurst, P.M. \& Bagley, S.K. Acute adaptation of the effects of alcohol. Quarterly Journal of Studies on Alcohol, 1972, 33, 358-378.

Hutchison, H.C., Tuchtie, M., Gray, K.G., \& steinberg, D.A. Study of the effects of alcohol on mental functions. Canadian Psychiatric Assoc. Journa1, 1964, 9, 33-42.

Idelstrom, C.M., \& Cadenius, B. Time solutions of the effects of alcohol compared to placebo. Psychopharmacologia, 1968, 13, 189-200.

Ikeda, H. , \& Granger, G.W. Action of alcohol on visual and retinal responses to intermittent illumination. In Alcohol and Road Traffic: Proceedings of the Third International Conference. London British Medical Association, 1963, 140-146.

Jakala, M., Rinkonen,. T.A., \& Markkanen, T. The effects of distilled and brewed beverages: A physiological, neurological and psychological study. Helsinki: The Finnish Foundation for Alcohol Studies, 1957, Publ. No. 4, 195.

Jansen, G. Experimental studies in the form of pharmacological performance experiments. Med. Exptl. Basel, 1960, 2, 209-216.

Jellinek, E.M., \& MCFarland, T.A. Analysis of psychological experiments on the effects of alcohol. Quarterly Journal of studies on Alcohol, 1940, 1, 272-279.

Jennings, J.R., Woods, C.C., \& Lawrence, B.E. Effects of graded doses of alcohol on speed-accuracy tradeoff in choice reaction time: Perception and Psychophysics, 1976, 19, 85-91.

Joerger, K. Das Erleben der Zeit and seine Veranderung durch Alkoholeinfuss; Eine untersuchung uber den Arbeitscharakter von Schulem an weinorten. (The experience of time and its change under the influence of alcohol; an investigation of the manner of working by school children in viticultural regions.) z. Ezptl. Angew. Pscychol., 1960, 7. 126-161. (Abstract, Quarterly Journal of Studies in Alcohol, $1963,24,545-546$.

Jones, B.M. Alcohol and memory impairment: A reinterpretation of the dose-response phenomonen. Biological Psychology Bullentin, 1973, 3. $2-8$.

Jones, B.F., Flinn, R.H., \& Hammond, E.C. Fatigue and hours of service of intertate truck drivers. U.S. Public Health Service Bulletin No. 265, 1941: 
Jones, R.K., Joscelyn, K.B. Alcohol and Highway Safety 1978: A Review of the State of Knowledge. Final Report, DOT HS-5-01217, 1978.

Joyce, C.R.B., Edgecombe, P.C.E., Kennard, D.A., Weatherall, M., \& Woods, D.P. Potentiation by phenobarbitane of effects of ethyl alcohol on human behavior. Journal of Mental Science, 1959, $105,51-60$.

Kahneman, D. Attention and Effort. Englewood Cliffs, New Jersey: Prentice Hal1, 1973.

Kaluger, N.A., \& Smith, G.L. Driver eye-movement patterns under conditions of prolonged driving and sleep deprivation. Highway Research Record, 1970, 336, 92-106.

Kelley, M., Myrsten, A.L., Neri, A., \& Rydberg, U. Effects and aftereffects of alcohol on physiological and psychological functions in man: A controlled study. Blutalkohol, 1970, ㄱ, 442-36.

Kennedy, R.S. The relationship between vigilance and eye movements inducted by vestibular stimulation. In Mackie, 1977.

King, A. Tunnel vision. Quarterly Journal of Studies on Alcohol, 1943, 4. 362-367.

Kinsman, K.A., \& Weiser, P.C. Subjective symptomatology during work and fatigue. In Simonson E. and Weiser, P.C. (Eds.), Psychological Aspects and Physiological Correlates of Work and Fatigue. Springfield, Ill.: Charles C. Thomas, 1976.

Kinsman, R.A., Weiser, P.C., \& Stamper, D.A. Multidimensional analysis of subjective symptomatology during prolonged strenuous exercise. Ergonomics, 1973, 16, 211 .

Kleitman, N. Sleep and Wakefulness. Chicago, Illinois: University of Chicago press, 1963.

Kogi, K., Saito, Y. \& Mitsuhashi, T. Validity of three components of subjective fatigue feelings. Journal of science of Labor, 1970 , 46, 251-256.

Kristozov, K.H, \& Atsev, E. Comparative study of the bioelectric activity of the brain and higher nervous activity in acute alcohol intoxication. Quarterly Journal of Studies on Alcohol, 1963, 24. 547, (Abstract).

Landauer, A.A., \& Milner, G. Desipramine and imipramine, alone and together with alcohol in relation to driving safety. Pharmakopsychiatrie Neuro-Psychopharmakologie, 1971, 4, 265-275.

Lange, J., \& Specht, W. Neve Untersuchungen uver die Beeinflussing der Sinnesfunktionen durch geringe Alkoholmengen. $\underline{z}$. Pathopsyche, 1915, 3. 155-265. (As cited in Jellinek and McFarländ, 1940). 
Lisper, H.O., Dureman, I., Ericsson, S., \& Karlsson, N.G. Effects of sleep deprivation and prolonged driving on a subsidiary auditory reaction time. Accident Analysis and Prevention, 1971, 2, 335-341.

Loomis, T.A., \& West, T.C. The influence of alcohol on automobile driving ability. Quarterly Journal of Studies on Alcohol, 1958, 19, $30-46$.

Lovibond, S.H., \& Bird, K. Effects of blood alcohol level on the driving behavior of competition and non-competition drivers. Paper presented at $29 \mathrm{th}$ International Congress on Alcoholism and Drug Dependence, Sydney, Australia, February, 1970.

Lowenstein, 0 . Der psychische Restitutionseffekt. Das Prinzip der psychischen Wiederher stellung der Ermudeten, der Erschopften und der Erkrankten Funkiton, Basel. Germany: Benno Schwabe and Co., 1937 (Cited in Bartley and Simonson, 1976).

Lowenstein, $0 ., \&$ Lowenfeld, L.E. Disintegration of central autonomic regulation during fatigue and its reintegration by psychosensory controlling mechanisms, disintegration, reintegration, pupillographic studies. Journal of Nervous and Mental Disease, $1952,115,121-145$.

Lubin, R.A. Influences of alcohol upon performance and performance awareness. Perceptual and Motor Skills, 1977, 45, 303-310.

Mackie, R.R. (Ed). Vigilance. New York: Plenum Press, 1977.

Mackie, R.R., \& O'Hanlon, J.F. A study of the combined effects of extended driving and heat stress on driver arousal and performance. In Mackie, 1977 .

Mackworth, N.H. Researches in the measurement of performance. Medical Research Council, Special Report, No. 268, 1950.

Mackworth, N.H., \& Morandi, A.J. The gaze selects informative details within pictures. Perception and Psychophysics, 1967, 2, 547-552.

Mackworth, J.F. Vigilance and Attention: A Signal Detection Approach. Middlesex, Hammondsworth: 1970 .

Marquis, D.B., Kelly, E.L., Miller, J.G., Gerord, R.W., \& Rapoport, A. Experimental studies of behavioral effects of neprobamate on normal subjects. Annals of the New York Academy of Sciences, 1957, 67, $701-711$.

Martin, G.L. The effects of small doses of alcohol on a simulated driving task. Journal of Safety Research, 1971, 3, 21-27. 
Martin, R., LeBreton, R. \& \& Roche, M. Variations individuelles du comportement sous l'influence de doses moyennes d'alcohol

ethylique. (Individual variations in behavior under the influence of moderate doses of ehtyl alcohol). Annals of Medical Psychology. $1957, \underline{37}, 56-70$.

Mast, T.M., Jones, H.V., \& Heimstra, N.W. Effects of fatigue on performance in a driving device. Highway Research Record, 1966, presented in $1965, \underline{122}, 93$.

McCarroll, J.R., \& Haddon, W., Jr. A controlled study of fatal automobile accidents in New York City. Journal of Chronic Diseases, $1962,15,811-826$.

McCoy, w. Going to sleep for good. The California Highway Patrolman, $1974,37,6-7$.

McFarland, R.A., \& Mosely, A.L. Human factors in highway transport safety. Boston, Mass.: Harvard School of Public Health, 1954 .

Mclellan, D.R. The effects of alcohol on driving skill. Highway Safety Literature, 1969, 3, 193-202.

McLelland, D.C., David, W.N., Kalin, R., \& Warner, E. The drinking man. New York: Free Press, 1972.

MCNelly. G. The development and laboratory validation of a subjective fatigue scale. Unpublished doctoral dissertation, Purdue University, 1954.

Michaut, G. \& Pottier, M. Conduite en situation monotone. (Driving in monotonous situations.) Organisme Nationale de Securite Routiere Bulletin, No. 8, 1964.

Mortimer, T.G. Effects of low blood alcohol concentrations in simulated day and night driving. Perceptual and Motor Skills, 1963, 17, 399-408.

Mortimer, R.G., \& Sturgis, S.P. Some effects of alcohol on car driving on two-lane and limited-access highways. Proceeding of the Human Factors Society 23rd Annual Meeting, 1979, 254-258.

Moskowitz, H. Laboratory Studies of the effects of alcohol on some variables related to driving. Journal of Safety Research, 1973, 5, 185-199.

Moskowitz, H., \& Burns, M. The effect of alcohol upon the psychological refractory period. Quarterly Journal of studies on Alcohol, $1971,32,782-790$. 
Moskowitz, H., \& Depry, D. The effect of alcohol upon auditory vigilance and divided attention tasks. Quarterly Journal of studies on Alcohol, 1968, 29, 54-63.

Moskowitz, H., \& Keller, M. Paper presented to American Psychological Association Convention, New York, September, 1979. (Cited in Moskowitz and Austin, 1979).

Moskowitz, H., \& Murray, J. Alcohol and backward masking of visual information. Quarterly Journal of Studies on Alcohol, 1976, 37, 40-45.

Moskowitz, H., \& Roth, S. The effect of alcohol upon the latency in naming objects. Quarterly Journal of Studies on Alcohol, 1971 , 32, 969-975.

Moskowitz, H., \& Austin G. A review of selected research studies from the last decade on the effects of alcohol on human skills perperformance. In Wm. Landry (Ed.), A Critical Review of the Drug.

Moskowitz, H., \& Sharma, S. Effects of alcohol on peripheral vision as a function of attention. Human Factors, 1974, 16, 174-180.

Moskowitz, H., \& Zeidman, K. Eye movements and skills performance measures under alcohol in a driving simulator. Proceedings of the Human Factors Society, 23rd Annual Meeting, 1979, 389-393.

Moskowitz, H., Zeidman, K., \& Sharma, s. Visual search behavior while viewing driving scenes under the influence of alcohol and marihuana. Human Factors, 1976, 18, 417-432.

Muller, B.P., Tarpey, R.D., Georgi, A.P., Mirone, L., \& Rouke, F.L. Effects of alcohol and mephenoxalone on psychophysiological test performance. Dis Nerv. Syst., 1964, 25, 373-375.

Murrel1, K.F.H. Ergonomics: Man in his working environment. London: Chapman and Hali.1965.

Muscio, B. Is a fatigue test possible? British Journal of Psychology, 1921, 12, 31-46.

Nash, H. Alcohol and caffeine: A study of their psychological effects. Charles C. Thomas, Springfield, I11., 1962.

Nash, H. Psychological effects and alcohol antagonizing properties of caffeine. Quarterly Journal of Studies on Alcohol, 1966, 27, $727-734$.

National Highway Traffic Safety Association. Alcohol safety action projects evaluation methodology and overall program impact-Vol. 3. DOT-HS-803-896, April 1979. 
Neilsen, R.A. Alcohol involvement in fatal motor vehicle accidents. California, 1962-1968. San Francisco: California Traffic Safety Foundation, 1969.

Nelson, T.M., Ladan, C.J., \& Carlson, D. Perceptions of fatigue as related to alcohol ingestion. Waking and sleeping, 1979, 3, 115-135.

Newman, H.W. The effect of attitude on alcohol tolerance. Quarterly Journal of Studies on Alcohol. 1949, 10, 398-403.

Newman, H., \& Fletcher, E. The effect of alcohol on vision. American Journal of Medical Science, 1941, 202, 723-731.

Newman, H., Fletcher, E., \& Abramson, M. Alcohol and driving. Quarterly Journal of Studies on Alcohol, 1942, 3, 15-30.

Noton, D., \& Stark, L. Scanpaths of saccadic eye movements while viewing and recognizing patterns. Vision Research, 1971, 11, 929942 .

Nunney, D.N. Fatigue, impairment, and psycho-motor learning. Perceptual and Motor Skills, 1963, 16, 369-375.

Obitz, F.W., Rhodes, L.E., \& Creel, D. Effect of alcohol and monetary reward on visually evoked potentials and reaction time. Journal of Studies on Alcohol, 1977, 38, 2057-2064.

O'Hanlon, J.F., \& Kelley, G.R. Comparison of performance and physiological changes between drivers who perform well and poorly during prolonged vehicular operations. In R.R. Mackie (Ed.), 1977.

O'Hanlon, J.F., \& Beatty, J. Concurrence of electro-encephalographic and performance changes during a simulated radar wacth. In $R . R$. Mackie, 1977.

O'Hanlon, J.F. Heart rate variability: A new index of driver alertness/fatigue. Goleta, California: Human Factors Research, Inc.,

Ohkubo, T. Psycho-physiological reactions of vehicle drivers under long distance driving. Proceedings, 6th Congress of International Ergonomics Association, University of Maryland, 1976. (Cited in Shinar et al., 1978.)

Oklahoma Turnpike Authority Press Release (as cited in Case Hulbert, $1970)$.

Olsen, R.A., \& Post, D.L. Fatigue and performance. Report \# PTI 7904. University Park, PA. The Pennsylvania Transportation Institute, Penn. State University, 1979. 
Pearson, R.G. Alcohol-hypoxia effects upon operator tracking, monitoring, and reaction time. Aerospace Medicine, 1968, 39 ,

303-307.

Pearson, R.G., \& Byars, Jr., G.E. The development and validation of a checklist for measuring subjective fatigue. United States Air Force School of Aviation Medicine Report, No. 56-115, 1956.

Peeke, S.C., Calloway, E., Jones, R.T., Stone, G.C., \& Doyle, J. Combined effects of alcohol and sleep deprivation in normal young adults. Psychopharmacology, 1980, 67, 279-288.

Perrine, M.W. Alcohol influences of driving related behavior: A critical review of laboratory studies of neurophysioloical, neuromuscular and sensory activity. Journal of Safety Research, $1973,5,165-184$.

Perrine, M.W., \& Huntley, M.S., Jr. Influences of alcohol upon driving behavior in an instrumented car. (NHTSA Technical Report, DOT HS-800-47I) Washington, D.C.: U.S. Department of Transportation, February, 1971 .

Perrine, M.W., Waller, J.A., \& Harris, L.S. Alcohol and Highway safety: Behavioral and medical aspects. NHTSA, Technical Report, DOT-HS-800-599, 1971 .

Peters, H.B. Changes in color fields occasioned by experimentally induced intoxication. Journal of Applied Psychology, 1942, 26, 692-701.

Pfeiffer, M.G., Siegel, A.I., Taylor, S.E., \& Shuler, L. Background data for the human performance in continuous operations guidelines. Technical Report \#386. U.S. Army Research Institute for the Behavioral and Social Sciences, 1979.

Pierson, W.R. Fatigue, work decrement, and endurance in a simple repetitive task. British Journal of Medical Psychology, 1963, 36, 279.

Platt, F. A new method of evaluating the effects of fatigue on driver performance. Human Factors, 1964,6 , 351-358.

Platt, F.N., \& Fedderson, G.J. Driver performance: The search for objective measurements. Presented at the Symposium and Workshop on the Suantification of Human Performance - Human Factors Society, August 17-19, 1964.

Poffenberger, A.T. The effects of continuous work upon output and feelings. Journal of Applied Psychology, 1928, 12, 459.

Powell, W.H., Jr. Ocular manifestations of alcohol and a consideration of individual variations in seven cases studied. Journal of Aviation Medicine, 1938, 9, 47-103. 
Prag, J.J. The chemical and clinical diagnosis of "driving under the influence of alcohol" and the use of the chemical tests in traffic law enforcement. South African Journal of Clinical Sciences, 1953, 4. 289-325 (cited in Wallgren and Barry, 1970).

Rackoff, N.S., \& Rockwell, T.H. Driver search and scan patterns in night driving. In Driver Visual Needs in Night Driving. Washington, D.C., Transportation Research Board, Special Report 156, 1975, 5363.

Rafaelsen, O.J., Bech, P., Christiansen, J., Christrop, H., Nyboe, J., * Rafaelsen, L. Cannabis and alcohol: Effects on simulated car driving. Science, 1973, 179, 920-923.

Rafaelsen, O.J., Bech, P., \& Rafaelsen, J.. Simulated car driving influenced by cannabis and alcohol. 'Pharmakopsychiatrie Neuropsychopharmokologie, 1973, 6, 71-83.

Rafaelsen, L., Christup, H., \& Bech, P. Effects of cannabis and alcohol on psychological tests. Nature, 1973, 242, 117-118.

Rauschke, J. Tests of performance during rising and falling blood alcohol levels under special conditions. Deut, Z. Ges. Gerichtl. Med., 1954, 43, 27-37. (cited in Wallgren and Barry, 1970).

Reid, L., Hansteen, R.W., \& Miller, R.D. The effects of cannabis and alcohol on psychomotor tracking performance. Unpublished report summarized in Cannabis, A Report of the Commission of Inquiry into the Non-Medical Use of Drugs. Ottawa: Information Canada, 1972 .

Reid, L.D., \& Ibraham, M.F. The application of human operator describing functions to studies on the effects of alcohol and marijuana on human performance. IEEE Transactions on systems, Man and Cybernetics, 1975, 5, 506, 519.

Reisby, W., \& Theilgaard, A. The interaction of alcohol and meprobamate in man. Acta Psychiatrica Scandinavica, 1969, Suppl. 208.

Riemersma, J.B.J., et al. Performance decrement during prolonged night driving. In Mackie, 1977.

Rockwell, T.H. Eye movement analysis of visual information acquisition in driving: An overview paper No. 948. Proceedings of the 6th Conference Australian Road Research Board, $197 \overline{2}, \underline{6}, 316-331$.

Rokseth, R., \& Lorentzen, F.W. Combined effect of alcohol and hypoxia on flicker fusion frequency. Journal of Applied Physiology, 1954, 6 , 559-565. 
Romansky, M.L., Plummer, R.W., \& Newmann, E.S. Environmental stressors as causal factors for driver fatigue. Proceedings of the Human Factors Society, 23rd Annual Meeting, 1979, 272-276.

Romansky, M.L. Stress and fatigue in the driving environment. Unpublished doctoral dissertation, West Virginia University, 1978.

Rosenthal, V. The influence of alcohol practice on learning a visualmotor skill. Doctoral Diss.. Illinois Institute of Technology, Chicago, 1959. (Abstr. Quarterly Journal of Studies on Alcohol, $1959,20,369$.

Rubin, A., Poparia, N., \& McMichael, A. Some effects of alcohol on Rorscharch performance. Journal of Clinical Psychology, 1954, 10, 252-255.

Rutenfrantz, J., \& Jansen, G. The compensation of the alcohol effect by caffeine and pervitin in a psychomotor performance. Internationale Zeitschrift fur Angewandte Physlogie Eienschliesslich Arbeitphysiologie (Berlin), 1959, 18, 62-81.

Rutschmann, J., \& Rubenstein, L. Time estimation, knowledge of results and drug effects. Journals of Psychiatric Research, 1966, 4. 107-114.

Ryback, R. The continuum and specificity of the effects of alcohol on memory. Quarterly Journal of Studies on Alcohol, 1971, 32, 9951016.

Safford, R.R., \& Rockwell, T.H. Performance decrement in twentyfour hour driving. Highway Research Record, No. 163, 1967.

Saito, Y., Kugi, K., \& Kashiwagi, S. Factors underlying subjective feelings of fatigue. Journal of Science of Labor, 1970, 46, 205.

Sanders, J.H. Driver performance in countermeasure development at railroad-highway grade crossing. Transportation Research Record, $1976,562,28-37$.

Schmidtke, H. Die Ermudung: Symptome, Theorie Messversuche. Stuttgart, Hans Huber, 1965. (cited in Bartley, 1976).

Seehafer, R.W., Huffman, W.K., \& Kinzie, M.D. Effects of low level blood alcohol concentration on psychophysiological and personality measures under controlled driving conditions. National Safety Congress Transactions, 1968, 23, 100-107.

Sekular, R., \& MacArthur, R.D. Alcohol retards visual recovery from glare by hampering target acquisition. Nature, 1977, 270, $428-429$. 
Shillito, M., King, L., \& Cameron, C. Effects of alcohol on choice reaction time. Quarterly Journal of Studies on Alcohol, 1974, 35, 1023-1034.

Shinar, D., Zaidel, D.M., \& Paarlberg, W.T. Driver performance and individual differences in attention and information processing. Vol. 1: Driver Inattention. DOT-HS-801819. Institute for Research in Public Safety, Bloomington, Ind., 1978.

Shinar, D. Psychology on the road. Wiley \& Sons, New York, 1978.

Shinar, D. Driver vision and accident involvement: New findings with new vision tests. In Proceedings of the American Association for Automotive Medicine (22nd Conference) and the International Association for Accident and Traffic Medicine (VII Conference), July 10-14, 1978, Ann Arbor, Michigan, Vol. 1, ed. D.F. Huelke, pp. 81-91. Morton Grove, Illinois: American Association for Automotive Medicine.

Shinar, D., McDowe11, E.D., \& Rockwell, T.H. Improving driver performance on curves in rural highways through perceptual changes. The Ohio State University, Engineering Experiment Station, Report EES428B 1974 .

Sidell, F.R., \& Pless, J.E. Ethyl alcohol: Blood levels and performance decrements after oral administration to man. Psychopharmacdcogia, 1971, 19, 246-261.

Simonson, E., \& Weiser, P.C. (Ed's) Psychological Aspects and Physiological Correlates of Work and Fatigue. Springfield, Illinois: Charles C." Thomas, 1976.

Simonson, E., \& Enzer, V. Measurement of fusion frequency of flicker as a test for fatigue of the central nervous system. Journal of Industrial Hygiene and Toxicology, 1941, 23, 83-89.

Sioux Falls Safety Council. A study of the effect of moderate blood alcohol levels on driving and driving-related functions. South Dakota Journal of Medical Pharmacology, 1963, 16, 27-30.

Sjolberg, L. Alcohol and gambling. Psychopharmacologia, 1969, 14, 284-298.

Skaglund, C.K. On the influence of alcohol on the pupillar, light reflex in man. Acta Physiologica Scandinavica, 1943, 6, 94-96.

Snapper, K.J. Effects of alcohol on psychomotor skill and decisionmaking in a driving task. Doctoral dissertation, University of Michigan, Ann Arbor, 1973, University Microfilms.

Soliday, S.M. Relationship between age and hazard perception in automobile drivers. Perceptual and Motor Skills, 1974, 39, 335-338. 
Starck, H.J. Studies on the traffic safety of drivers habituated to alcohol at blood alcohol concentrations around $1.5 \mathrm{mil}$. Deutsche Zeitschrift fuer die Gesampte Gerichtliche medizin, 1953, $42,155-161$ (cited in Wallgren \& Barry, 1970. )

Sterzinger, D. Chemopsychologische Untersuchungen uber den Zeitsinn. s. Dsychol., 1953, 124, 100-131. (Cited in Wallgren and Barry, 1970).

Sugarman, R.C., \& Cozad, C.P. Road tests of alertness variables. Final Report. DOT-HS-053-1-145, 1972.

Sugarman, R., Cozad, C., \& Zavala, A. Alcohol induced degradation of performance on simulated driving tasks. SAE Paper 730099, January, 1973.

Sussman, E.D., \& Morris, D.F. An Investigation of Factors Affecting Driver Alertness, FH-11-7313, DOT-HS-800-317. Buffalo, N.Y.: Cornell Aeronautical Laboratory of Cornell University, 1970.

Sutton, D., \& Burns, J. Alcohol dose effects on feedback-maintained simple reaction time. Journal of Psychology, 1971, 78, $151-159$.

Suzumura, A. Studies on kinetic visual acuity. The importance of the kinetic visual acuity as an ability of pilot. Annual Report of the Research Institute of Environmental Medicine, 1963, 11, 9-18. Japan: Nagoya University.

Suzumura, A. Studies on the kinetic visual acuity. Annual Report of the Research Institute of Environmental Medicine, $1 \overline{962,} \overline{10}, \overline{7-31}$. Japan: Nagoya University.

Talland, G.A. Effects of alcohol on performance in continuous attention tasks. Psychosomatic Medicine, 1966, 27, Part II, 596-604.

Talland, G.A., Mendelson, J.H., \& Ryack, P. Experimentally induced chronic intoxication and withdrawal in alcoholics. Pt. 5. Tests of Attention. Quarterly Journal of Studies on Alcohol, Suppl. No. 2, 1964, 74-86.

Tarter, R.E., Jones, B.M., Simpson, C.D., \& Vega, D. Effects of task complexity and practice on performance during acute alcohol intoxication. Perceptual and Motor skills, 1971, 33, 307-318.

Taylor, J.D., \& Stevens, D.L. Dose response relationship of ethanol and automobile driving. Alcohol and Traffic safety proceedings of the Fourth International Conference on Alcohol and Traffic Safety, Indiana University, BIoomington, IN., Dec. 6-10, 1965. 
Teger, A.I., Katkin, E.S., \& Pruit, D.G. Effects of alcoholic beverages and their congener content on level and style of risk taking. Journal of Personality and Social Psychology, 1964, 11, 170-176.

Teichner, W.H. Recent studies of simple reaction time. Psychological Bulletin, 1954, 51, 128-149.

Teichner, W.H. Response blocking. A necessary performance criterion. In Pre-Crash Factors in Traffic Safety. American Association for Automotive Medicine, Proceedings, 12th Annual Symposium, 1968, 165-180.

Thackray, R.I., Bailey, J.P., \& Touchstone, R.M. Physiological, subjective, and performance correlates of reported boredom and Monotony while performing a simulated radar control task. In Mackie 1977.

Treat, J.R., Tumbas, N.S., McDonald, S.T., Shinar, D., Hume, R. D., Mayer, R.E., Stansifer, R.L., \& Castellan, N.J. Tri-level study of the causes of traffic accidents: Final report, Executive Summary. DOT-HS-034-3-535-79-TAC (s), Institute for Research in Public Safety, 1979.

Verriest, G. \& Laplasse, D. New data concerning the influence of ethyl alcohol on huyman visual thresholds. Experimental Eye Research, 1965,4 , 95-101.

Von Wright, \& Mikkonen. The influence of alcohol on the detection of light signals in different parts of the visual field. Scandinavian Journal of Psycholgogy, 1970, 11, 167-175.

Waller, J.A., King, E.M., Nielson, G., \& Turkel, H.W. Alcohol and other factors in California highway fatalities. In proceedings of the Eleventh Annual Meeting of the American Association for Automotive Medicine. Springfield, IL: Charles C. Thomas, 1970.

Wallgren, H. \& Barry, H. Actions of Alcohol (2 Volumes). Amsterdam: Elsevier, 1970 .

Walter, H.W. Alkoholmissbrauch undalkohollagenystagmus. Deut. Z. Ges. Gericht1. Med., 1954, 43, 232-241. (Cited in Wallgren and Barry, 1970.)

Welford, A.T. Fatigue and monotony. In O.G. Edholm (Ed.), The Physiology of Human Survival. Academic Press: New York, 1965, Chapter 15.

Weltman, G., \& Egstrom, G. Perceptual narrowing in novice drivers. Human Factors, $1966,8,499-506$. 
Wilkinson, R.T. The effect of lack of sleep on visual watch-keeping. Ouarterly Journal of Experimental Psychology, 1960, 7, 36-44.

Wilkinson, R.T. Interaction of lack of sleep with knowlerge of results, repeated testing, and individual differences. Journal of Experimental Psychology, 1961, 62, 263-271.

Wilkinson, R.T. Sleep deprivation, Chap. 14. In O.G. Edholm and A.L. Bacharach (Eds.). The Physiology of Human Survival. Academic

Press: London, $196 \overline{5 .}$

Wilkinson, R.T., \& Colquhoun, W.P. Interaction of alcohol with incentive and with sleep deprivation. Journal of Experimental

Psychology, 1968, 76, 623-629.

Williams, H.B., Personal communication. Alertmaster Company, Houston, TX, 1966. (Cited in Hulbert, 1972.)

Wilson, L., Taylor, J.D., Hash, C.W., \& Camerson, D.F. The combined effects of ethanol and amphetamine sulfate on performance on human subjects. Canadian Medical Association Journal, 1966, 94, 478-484.

Wolf, G. Construct validation of measures of 3 kinds of experimental fatigue. Perceptual and Motor Skills, 1967, 24, 1067-1076.

Wolff, H.G., Hardy, J.D. and Goodell, H. Studies on pain: Measurement of the effect of ethyl alcohol on the pain threshold and on the "alarm" reaction. Journal of Pharmacological Experimental Therapy, 1942, 75, 38-49.

Yajima, K., Ikeda, K., Oshima, M., and Sugi, T. Fatigue in automobile drivers due to long time driving. Warren, Mich.: Society of Automotive Engineers, Paper No. 76050, 1976.

Yoss, R.E. The sleepy driver: A test to measure ability to maintain alertness. Mayo Clinic Proceedings, 1969, 44, 769-783.

Yoss, R.E., Moyer, N.J., \& Hollenhorst, R.W. Pupil size and spontaneous waves associated with alertness, drowsiness, and sleep. Neurology, 1970, 20, 545-554.

Yudkin, S. Vitamin A and dark adaptation: Effect of alcohol, benzedrine an vitamin $C$. Lancet, 1941, 241, 787-791.

Zirkle, G.A., King, P.D., McAtee, O.B., \& Van Dyke, R. Effects of chlopromazine and alcohol on coordination and judgment. Journal of the American Medical Association, 1959, 171, 1496-1499.

zylman, R. Time of day as a factor in collision-involvement of drunken drivers. Quarterly Journal of Studies on Alcohol, 1973, 34, 527-529. 